\title{
The oxygen sensor PHD2 affects energy metabolism and cell function in macrophages
}

\author{
Doctoral Thesis \\ In partial fulfillment of the requirements for the degree \\ "Doctor of Philosophy (Ph.D.)“ \\ in the Molecular Medicine Study Program \\ at the Georg-August University Göttingen
}

submitted by

Annemarie Güntsch

born in München

Göttingen 2016 


\section{Members of the Thesis Committee:}

Supervisor:

Prof. Dr. Dörthe M. Katschinski

University Medical Center, Georg-August-University Göttingen

Institute of Cardiovascular Physiology

Humboldtallee 23

37073 Göttingen, Germany

Co-Supervisor:

Prof. Dr. Ajay Shah

The James Black Centre, King's College London

125 Coldharbour Lane

London SE5 9NU, United Kingdom

$2^{\text {nd }}$ member of the thesis committee:

Prof. Dr. Susanne Lutz

University Medical Center, Georg-August-University Göttingen

Institute of Pharmacology

Rober-Koch-Straße 40

37075 Göttingen, Germany

$3^{\text {rd }}$ member of the thesis committee:

Prof. Dr. Frauke Alves

Max-Planck Institute for Experimental Medicine and University Medical Center, GeorgAugust-University, Göttingen

Molecular Imaging in Oncology

Hermann-Rein-Straße 3

37075 Göttingen, Germany 


\section{Affidavit:}

I declare that my doctoral thesis entitled "PHD2 affects energy metabolism and cell function in macrophages" has been written independently with no other aids than quoted.

Göttingen, July 2016

Annemarie Güntsch 


\section{Table of Contents}

List of Abbreviations .........................................................................................................

List of Figures ................................................................................................................................................. IX

List of Tables: ........................................................................................................................................... XI

Abstract .............................................................................................................................

1. Introduction .............................................................................................................................................

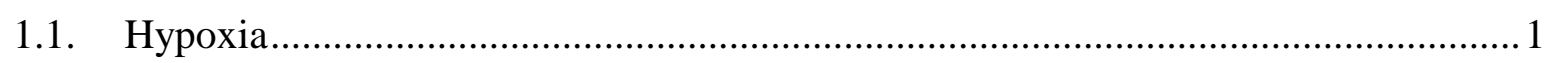

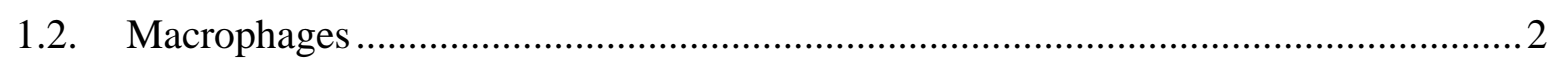

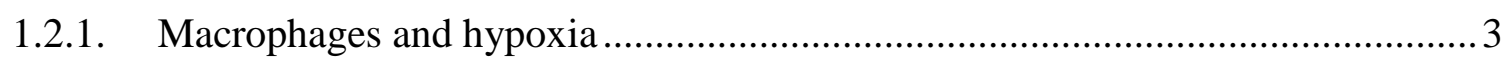

1.3. The Hypoxia-Inducible Factor (HIF) ..............................................................

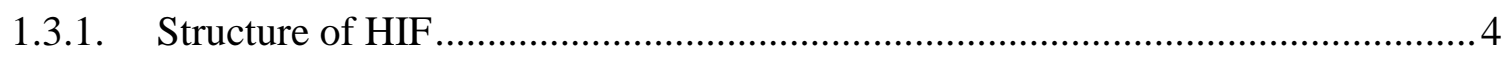

1.3.2. Regulation of HIFs by Prolyl-4-Hydroxylase Domain enzymes (PHD) and the Factor Inhibiting HIF (FIH) .............................................................................. 6

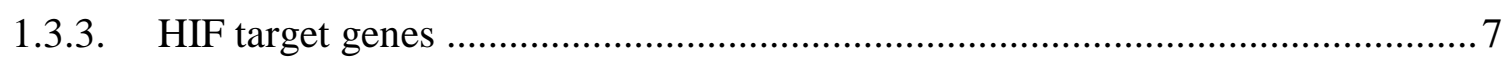

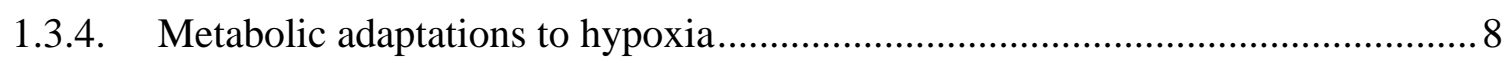

1.4. Prolyl-4-hydroxylase domain enzymes (PHDs) …………………………………..... 11

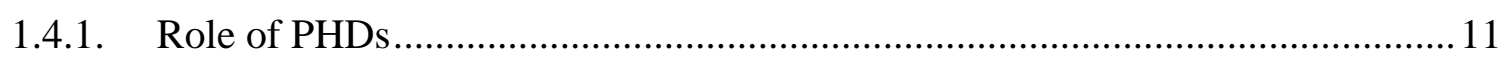

1.4.2. PHD hydroxylation reaction ....................................................................... 12

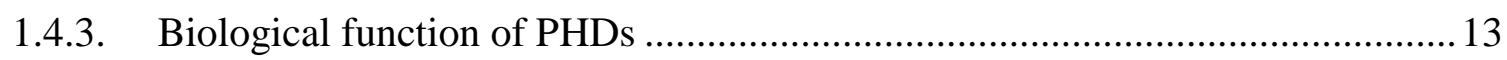

1.4.3.1. PHD1 genetically modified mouse models .......................................................... 13

1.4.3.2. PHD3 genetically modified mouse models …………………............................ 13

1.4.3.3. PHD2 genetically modified mouse models ....................................................... 14

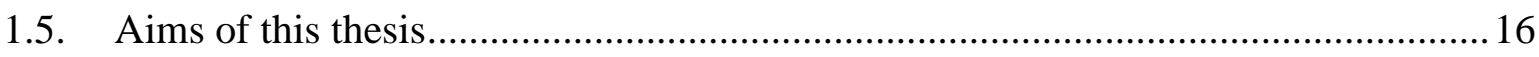

2. Material and Methods .....................................................................................................17

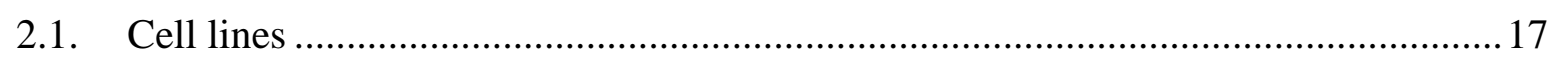

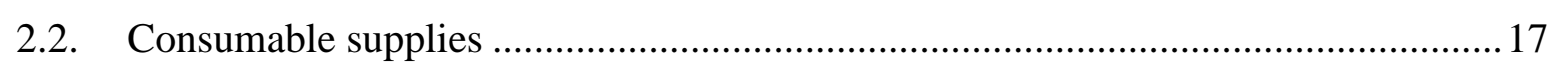

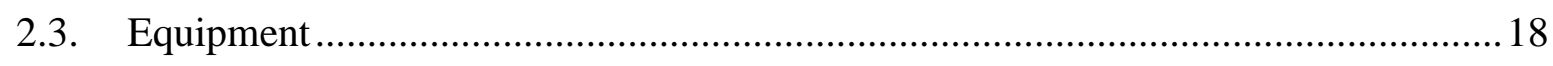

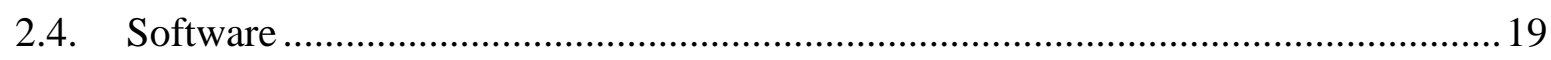




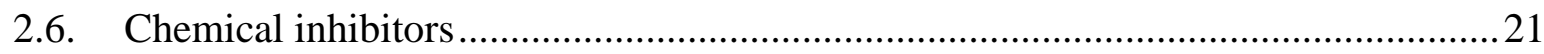

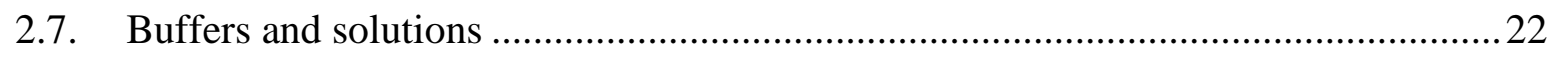

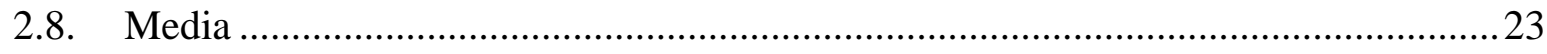

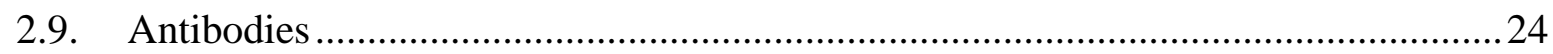

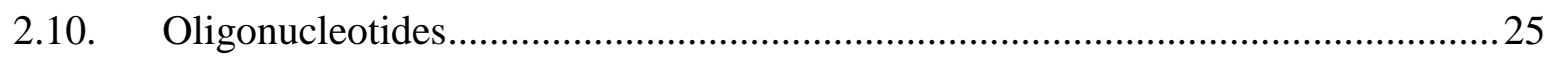

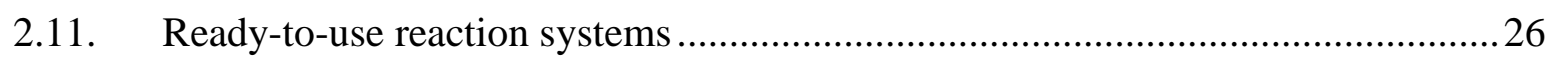

2.12. Mouse model .......................................................................................... 26

2.13. RAW cell line with constitutively active shRNA targeting PHD2 ....................27

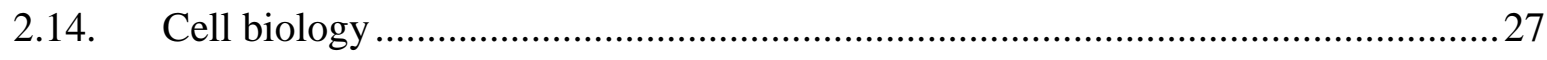

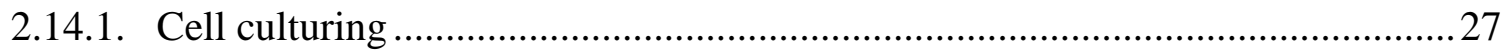

2.14.2. Preparation of MDA-MB 231 conditioned medium ....................................... 27

2.14.3. Preparation of L929 conditioned medium (Pluznik) ........................................ 28

2.14.4. Differentiation and cultivation of bone marrow-derived macrophages.............. 28

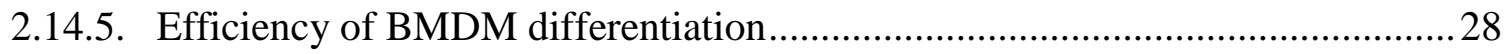

2.14.6. Isolation and cultivation of murine peritoneal macrophages..............................29

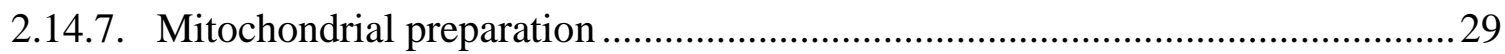

2.14.8. Determination of Lactate levels in BMDM and RAW supernatant ..................29

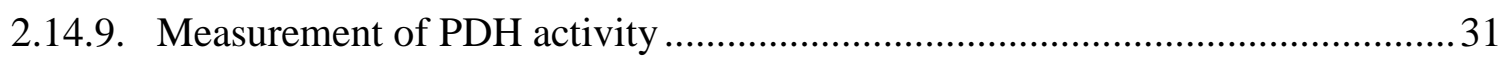

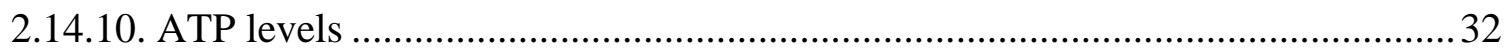

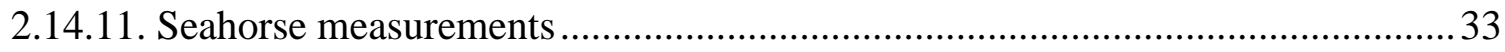

2.14.12. Measurement of phagocytosis by flow cytometry ........................................... 36

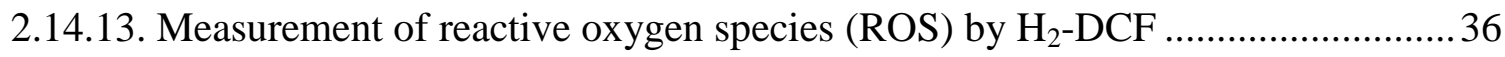

2.14.14. Measurement of reactive oxygen species by DHE-HPLC ................................ 37

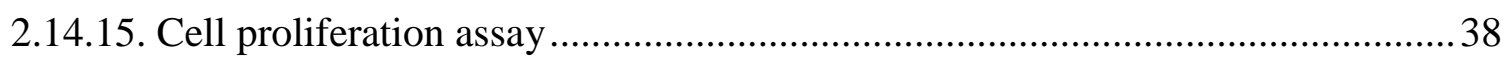

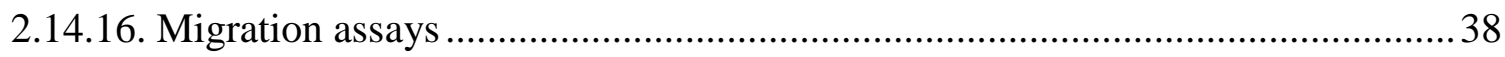

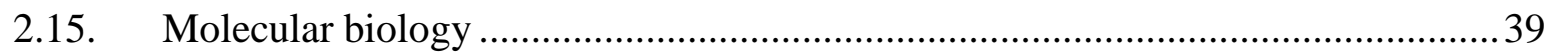

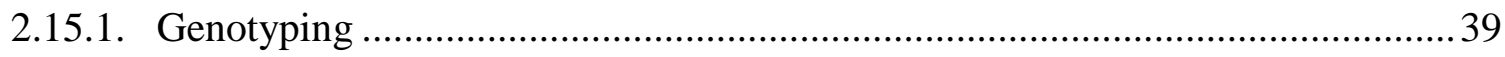

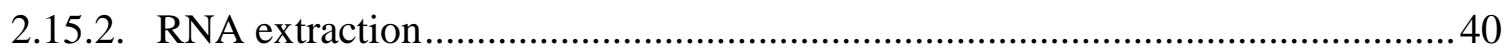

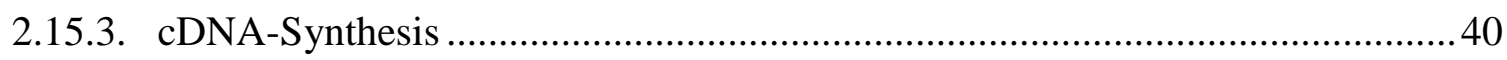


2.15.4. Transcript quantification by qRT-PCR (quantitative Real-Time-Polymerase Chain Reaction)

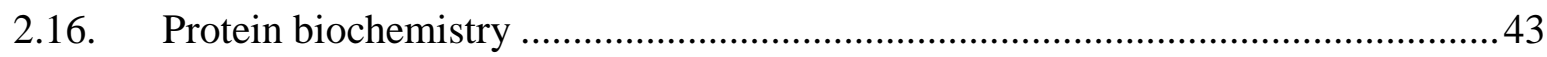

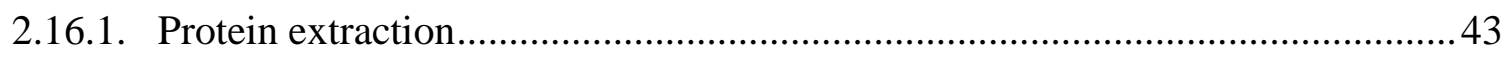

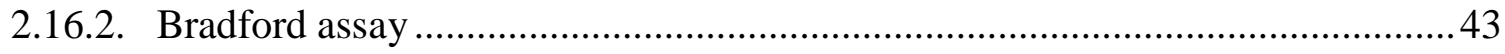

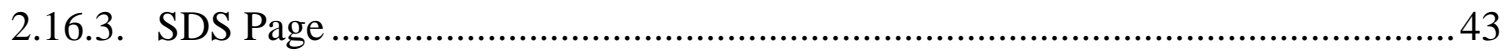

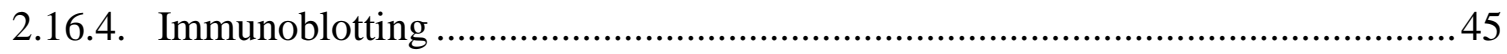

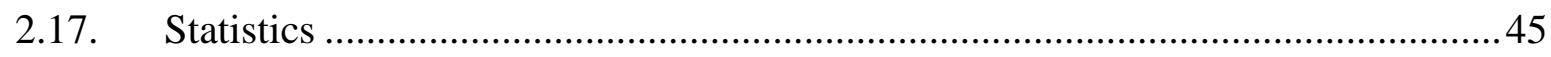

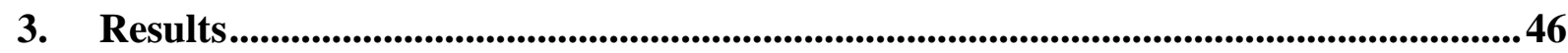

3.1. PHD2 deficient macrophages stabilize HIF-1 $\alpha$ and show a HIF target gene

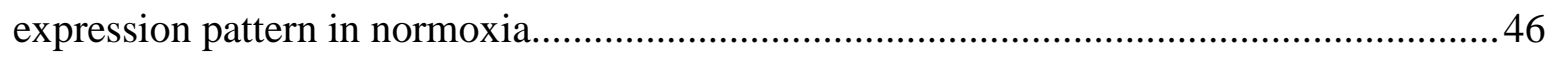

3.2. Migration and phagocytosis capacity is altered in PHD cKO and shPHD2 cells......50

3.2.1. PHD2 deficient macrophages migrate less than wt macrophages ......................50

3.2.2. Phagocytosis is impaired in shPHD2 RAW and PHD2 cKO macrophages.......53

3.2.1. Cell proliferation is not affected by PHD2-deficiency in RAW cells ................54

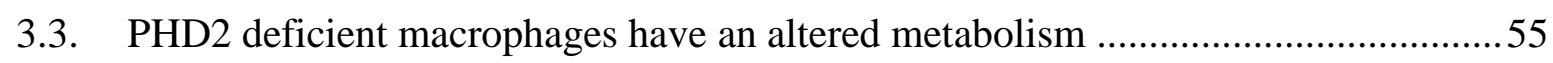

3.3.1. ATP levels are reduced in PHD2 cKO and shPHD2 macrophages...................55

3.3.2. Oxygen consumption rate is altered in PHD2 cKO and shPHD2 cells .............56

3.3.3. Extracellular acidification rate is increased in PHD2 cKO and shPHD2 cells ..58

3.3.4. Lactate levels are increased in PHD2 deficient macrophages ...........................59

3.3.5. Mitochondrial content of PHD2 deficient macrophages is unaltered..................60

3.3.6. Inhibition of mitochondrial respiration decreased migration .............................61

3.3.7. Mitochondrial ROS production is decreased in shPHD2 RAW cells ................62 62

3.3.8. Overall ROS production is increased in shPHD2 RAW cells ............................63

3.4. The metabolic alterations observed in PHD2 deficient macrophages depend on

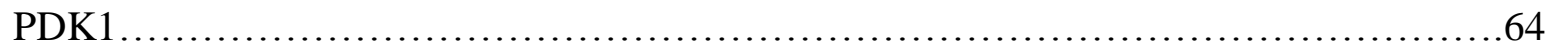

3.4.1. PDK1 protein levels are increased in PHD2 deficient macrophages .................64 64

3.4.2. PHD2 deficient macrophages show a decreased activity of PDH.....................65

3.5. PDK inhibition by dichloroacetate (DCA) partly restores the metabolic alterations in

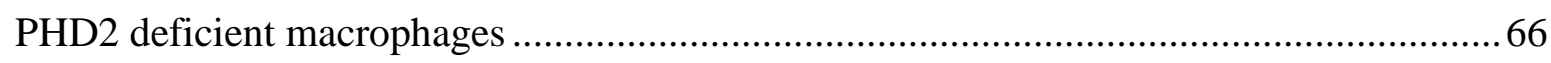

3.5.1. PDH activity in shPHD2 cells is restored by dichloroacetate treatment ............66 


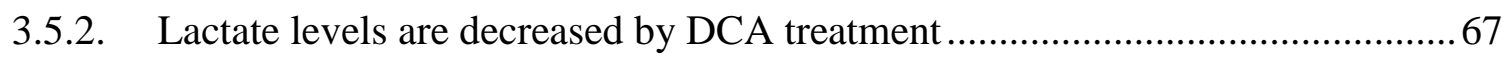

3.5.3. DCA reduces glycolysis in PHD2 deficient macrophages ................................ 68

3.6. DCA partially restores macrophage function in PHD2 deficient macrophages. ....... 70

3.6.1. Single cell migration in shPHD2 cells can be restored by DCA treatment ........ 70

3.6.2. Migration in a Boyden chamber assay is not affected by DCA treatment ......... 71

3.6.3. DCA treatment increases phagocytosis in PHD2 deficient macrophages .......... 71

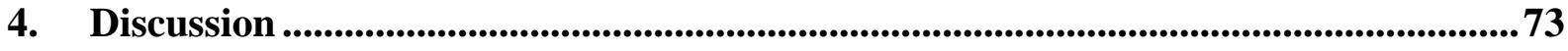

4.1. HIF-target genes are differentially expressed in PHD2 cKO and shPHD2 RAW

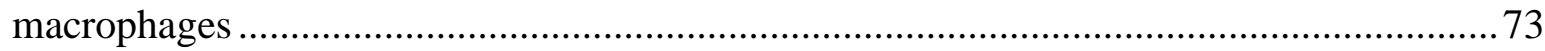

4.2. Macrophage function is altered in PHD2 deficient macrophages ...........................75

4.2.1. Migration and phagocytosis is impaired in PHD2 $\mathrm{cKO}$ and shPHD2

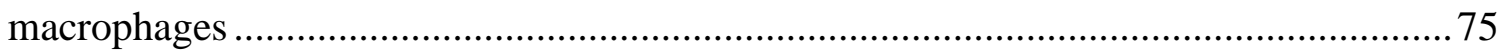

4.2.2. Treatment with DMOG or exposure to hypoxia also reduces migration and

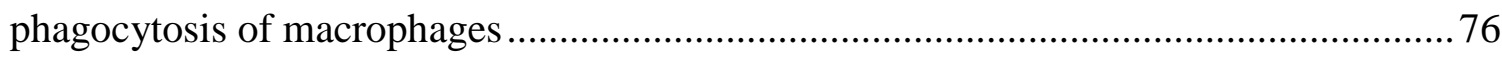

4.3. PHD2 deficiency in macrophages results in metabolic reprogramming ...................77

4.3.1. Macrophage energy metabolism is altered by PHD2 deficiency .......................77

4.3.2. ROS levels are differently altered in shPHD2 RAW isolated mitochondria and total cells

4.4. The metabolic alterations observed in PHD2 deficient macrophages depend at least partially on PDK1

4.5. Inhibition of PDK1 by DCA partially reestablishes wt phenotype in PHD2 deficient macrophages

4.6. PHD2 might provide an useful tool to influence macrophage metabolism and function.

5. Conclusion

References..

Vielen Dank! 103

Curriculum vitae Fehler! Textmarke nicht definiert. 


\section{List of Abbreviations}

\begin{tabular}{|c|c|}
\hline Abbreviation & Denotation \\
\hline$\%$ & percent \\
\hline${ }^{\circ} \mathrm{C}$ & degree Celsius \\
\hline$\mu$ & micro $\left(10^{-6}\right)$ \\
\hline $18 \mathrm{~S}$ & $18 \mathrm{~S}$ ribosomal RNA \\
\hline 2-DG & 2-deoxyglucose \\
\hline a.u. & arbitrary units \\
\hline ADP & adenosine diphosphate \\
\hline ANP & atrial naturetic peptide \\
\hline ARNT & aryl hydrocarbon receptor nuclear translocator \\
\hline Asn & asparagine \\
\hline ATG & start codon \\
\hline ATP & adenosine-triphosphate \\
\hline Bcl-2 & B-cell lymphoma-2 \\
\hline BMDM & bone marrow derived maxrophage \\
\hline BNIP3 & BCL/adenovirus E1B $19 \mathrm{kDa}$ interacting protein 3 \\
\hline BNP & B-type naturetic peptide \\
\hline BSA & bovine serum albumine \\
\hline CBP & cAMP response element-binding protein \\
\hline $\mathrm{CCCP}$ & carbonyl cyanide m-chlorophenyl hydrazone \\
\hline CCR & chemokine receptor \\
\hline CDC42 & cell division cycle 42 \\
\hline cDNA & copyDNA \\
\hline $\mathrm{cKO}$ & conditional knockout \\
\hline $\mathrm{CO} 2$ & carbon dioxide \\
\hline $\mathrm{CoA}$ & coenzyme A \\
\hline COX & cytochrome c oxidase \\
\hline CT & cycle threshold \\
\hline CXCR & CXC chemokine receptor \\
\hline $\mathrm{d}$ & days \\
\hline DCA & dichloroacetate \\
\hline DHE & dihydroethidium \\
\hline DMEM & Dulbeccos modified eagle medium \\
\hline DMOG & dimethyloxaloylglycine \\
\hline DNA & deoxyribonucleic acid \\
\hline dNTP & nucleoside triphosphate \\
\hline DPTA & diethylenetriaminepentaacetic acid \\
\hline ds & double-stranded \\
\hline $\mathrm{E}$ & embryonic day \\
\hline e.g. & exempli gratia \\
\hline ECAR & extracellular acidification rate \\
\hline ECL & enhanced chemiluminescence \\
\hline
\end{tabular}




\begin{tabular}{|c|c|}
\hline EDTA & ethylenediaminetetraacetic acid, \\
\hline EGTA & ethylene glycol-bis( $\beta$-aminoethyl ether)-N,N,N',N'-tetraacetic acid \\
\hline $\mathrm{EOH}$ & 2-hydroxyethidium \\
\hline EPAS & endothelial PAS domain protein \\
\hline EPO & erythropoietin \\
\hline et al. & latin: et alteri \\
\hline $\mathrm{EtOH}$ & ethanol \\
\hline FACS & fluorescence-activated cell sorting \\
\hline FCCP & carbonyl cyanide-4-(trifluoromethoxy)phenylhydrazone \\
\hline FCS & fetal calf serum \\
\hline $\mathrm{Fe}$ & iron \\
\hline Fig. & figure \\
\hline FIH & factor inhibiting HIF \\
\hline Fizz 1 & found in inflammatory zone 1 \\
\hline flox & floxed \\
\hline FSC & forward scatter \\
\hline g & gram \\
\hline GDP & guanosine diphosphate \\
\hline GLUT1 & glucose transporter 1 \\
\hline Glycostress medium & glucose stresstest medium \\
\hline GM-CSF & granulocyte macrophage-colony stimulating factor \\
\hline GPT & glutamate-pyruvate transaminase \\
\hline GTP & guanosine-5'-triphosphate \\
\hline $\mathrm{h}$ & hour \\
\hline $\mathrm{H}_{2} \mathrm{DCFDA}$ & dichlorodihydrofluorescein diacetate \\
\hline $\mathrm{H}_{2} \mathrm{O}$ & aqua dest \\
\hline $\mathrm{H}_{2} \mathrm{O}_{2}$ & hydrogen peroxide \\
\hline HEPES & 4-(2-hydroxyethyl)-1-piperazineethanesulfonic acid \\
\hline HIF & hypoxia inducible factor \\
\hline HLH & helix-loop-helix \\
\hline HPLC & high performance liquid chromatography \\
\hline HRE & hypoxia responsive elements \\
\hline HRF & HIF-related factor \\
\hline HRP & horseradish peroxidase \\
\hline $\mathrm{h}$ & hours \\
\hline i.e. & latin: id est \\
\hline IFN $\gamma$ & interferon $\gamma$ \\
\hline IL & interleukin \\
\hline iNOS & nitric oxide synthase \\
\hline KCL & potassium chloride \\
\hline $\mathrm{Km}$ & Michaelis constant \\
\hline KO & knockout \\
\hline $\mathrm{kPA}$ & kPascal \\
\hline $\mathrm{L}$ & liter \\
\hline LDH & L-lactate dehydrogenase \\
\hline
\end{tabular}




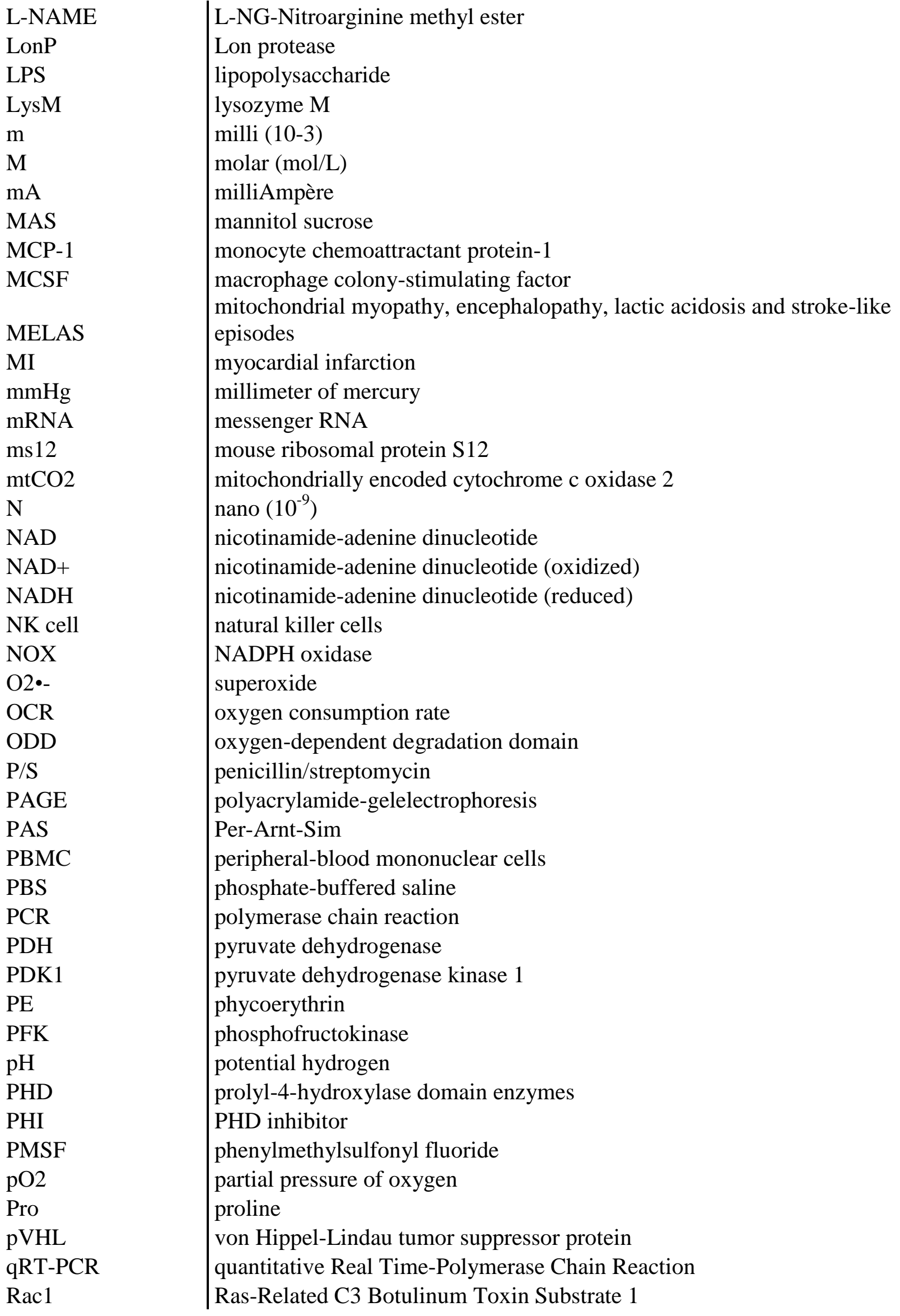




\begin{tabular}{l|l} 
RhoA & $\begin{array}{l}\text { Ras homolog gene family, member A } \\
\text { RNA }\end{array}$ \\
ROS & $\begin{array}{l}\text { reactive oxygen species } \\
\text { rounds per minute } \\
\text { room temperature }\end{array}$ \\
RT & $\begin{array}{l}\text { sodium dodecyl sulfate } \\
\text { standard error of the mean }\end{array}$ \\
SEM & serine \\
Ser & small hairpin \\
sh & small interfering RNA \\
siRNA & side scatter \\
SSC & time \\
T & transactivation domain \\
TAD & transactivation domain \\
TAD & Tris-buffered Saline Tween 20 \\
TBS-T & tricarboxylic acid cycle \\
TCA cycle & T-helper1 cells \\
Th1 cells & Trehalose HEPES EGTA \\
THE & temperature where 50\% of the helices are dissociated \\
Tm & tumor necrosis factor $\alpha$ \\
TNF $\alpha$ & Tris-hydroxymethyl-aminomethan \\
Tris & polyoxyethylensorbitanmonolaurat \\
Tween & units \\
U & ultraviolet \\
UV & Volt \\
V & volume percent \\
v/v & wildtype \\
VEGF & multiple acceleration of gravity $\left(\mathrm{g}=9.80665 \mathrm{~m} / \mathrm{s}^{2}\right)$ \\
wt & x
\end{tabular}




\section{List of Figures}

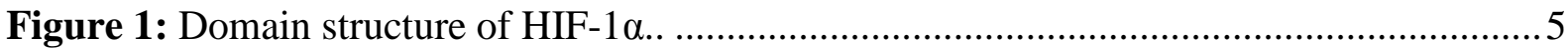

Figure 2: Summary of the regulation of HIF-1 $\alpha$ protein levels......................................... 7

Figure 3: Induction of metabolic adaptation by hypoxia-inducible factor (HIF) ................... 10

Figure 4: Hydroxylation reaction of prolyl-4-hydroxylases (PHD) ..................................... 12

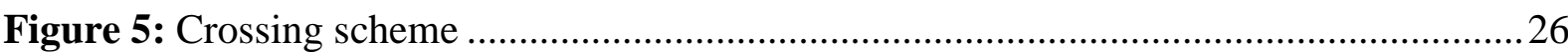

Figure 6: PHD2 protein levels are downregulated in shPHD2 cell and PHD2 cKO BMDMs.

Figure 7: PHD2 deficient RAW and BMDM macrophages show an induction of HIF target genes in normoxic conditions.

Figure 8: PHD2/HIF-1 $\alpha \mathrm{cKO}$ BMDMs have unchanged or downregulated gene expression levels of HIF target genes in normoxic conditions

Figure 9: A reduction of PHD2 expression in RAW cells or BMDMs results in a defect in macrophage migration.

Figure 10: Peritoneal macrophages isolated from PHD cKO mice results in a defect in cell migration

Figure 11: Migration of RAW wt cells is affected by DMOG and hypoxia exposure. .53

Figure 12: PHD2 deficient macrophages show a defect in phagocytosis. 54

Figure 13: Cell proliferation is unchanged in wt and shPHD2 RAW macrophages. 55

Figure 14: ATP levels are reduced in PHD2 deficient macrophages 56

Figure 15: Oxygen consumption rate is decreased in PHD2 deficient macrophages 57

Figure 16: RAW wt cells treated with DMOG have depressed oxygen consumption rate.....58

Figure 17: Extracellular acidification rate is increased in PHD2 deficient cells. 59

Figure 18: Lactate levels are increased in the supernatant of PHD2 cKO and shPHD2 RAW cells.

Figure 19: Mitochondrial content is unaltered in shPHD2 and cKO macrophages compared to the respective wt cells

Figure 20: Rotenone treatment decreases migration of RAW wt cells in single cell migration experiments 62

Figure 21: PHD2 affects mitochondrial ROS production. 63

Figure 22: HPLC analysis of DHE derived products in whole cell lysates of wt and shPHD2 RAW cells. 
Figure 23: PDK1 protein levels are increased in PHD2 cKO and shPHD2 cells. 65

Figure 24: PDH activity is decreased in shPHD2 cells. 66

Figure 25: PDH activity is increased after treatment with dichloroacetate (DCA)...............67

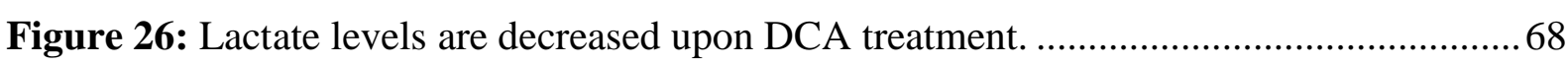

Figure 27: Extracellular acidification rate is reduced by DCA treatment. .............................69

Figure 28: DCA treatment increases migration in a single cell migration experiment. ..........70

Figure 29: DCA treatment does not affect migration in a Boyden chamber experiment........71

Figure 30: Phagocytosis is reestablished in DCA-treated PHD2 deficient macrophages ....... 72

Figure 31: PHD2 deficient macrophage undergo metabolic reprogramming dependent on PDK1 


\section{List of Tables:}

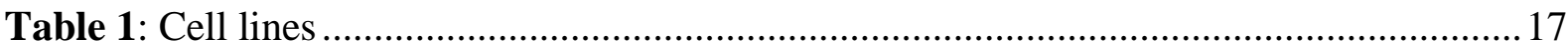

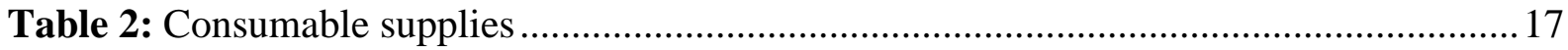

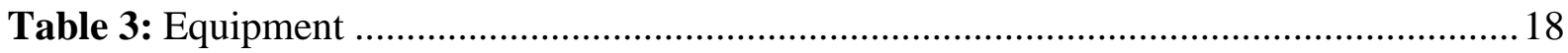

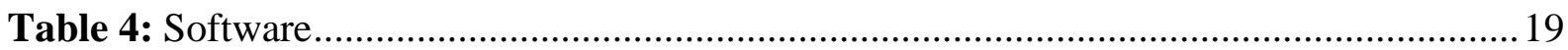

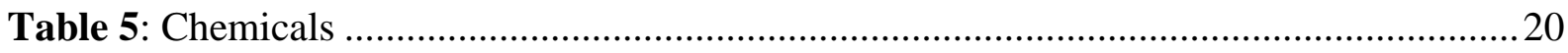

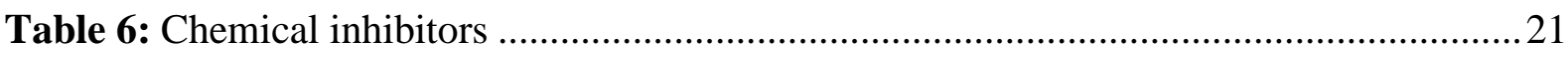

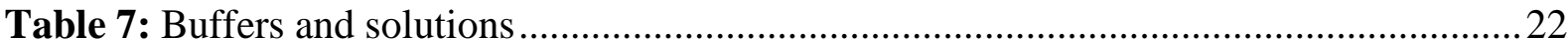

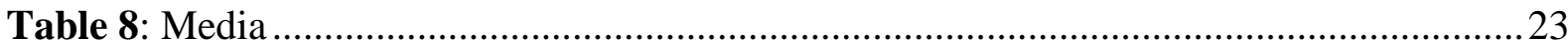

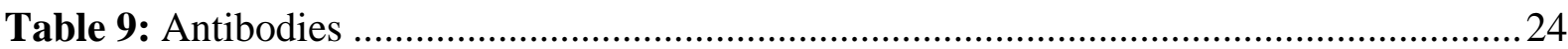

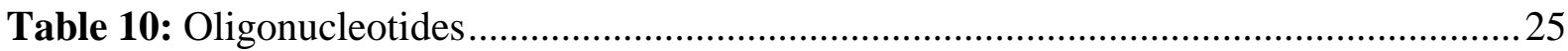

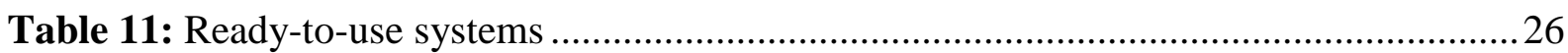

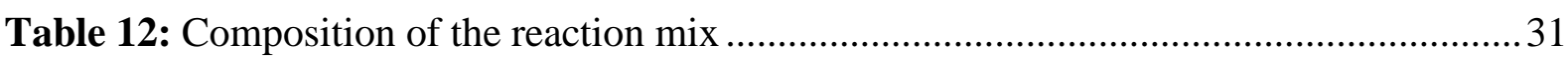

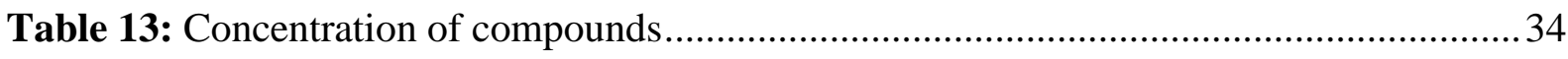

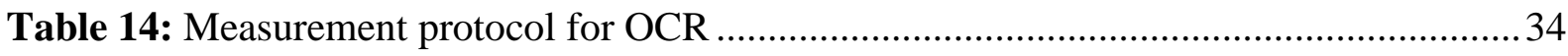

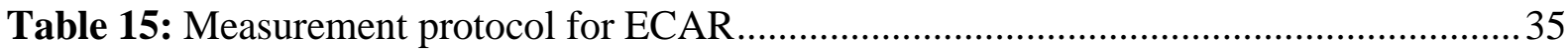

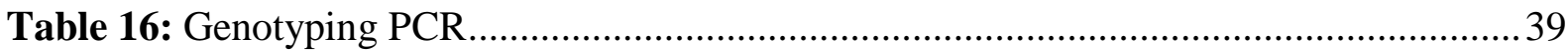

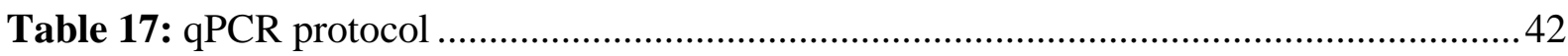

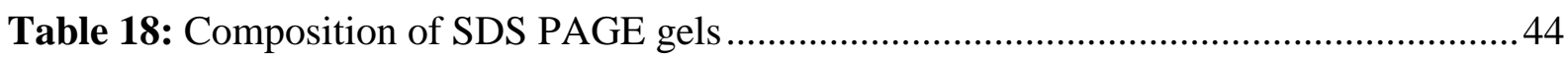




\section{Abstract}

Macrophages are key players in the innate immune system and contribute significantly to inflammation in ischemic tissues, such as the infarcted heart. During inflammation, macrophages are involved in tissue remodeling, which is needed for the reconstitution of the inflamed organ. Ischemic and inflamed tissues are characterized by a scarcity of oxygen. Macrophages migrate to the site of inflammation against the $\mathrm{O}_{2}$-gradient and therefore have to adapt rapidly to low oxygen concentrations. This adaptation is mediated by the hypoxia inducible factor (HIF)/prolyl-4-hydroxylase domain enzyme (PHD) pathway. The HIF- $\alpha$ subunit is hydroxylated by the three PHDs (PHD1-3) in an oxygen-dependent way. In normoxia, PHD2 has been described to be the main oxygen sensor. In hypoxia, HIF is stabilized and induces a specific gene expression programme. This ensures the survival of the cell in conditions of low oxygen availability.

I analyzed the impact of PHD2 on macrophage function using myeloid specific conditional PHD2 knockout mice (PHD2 $2^{\text {flox/flox }}$ LysMCre $^{+-}$) and a stably transduced RAW264.7 (RAW) macrophage cell line that express a PHD2 shRNA construct resulting in a knockdown of PHD2. In PHD2 deficient RAW cells and bone marrow derived macrophages (BMDMs), a normoxic stabilization of HIF-1 $\alpha$ was observed. The expression of the HIF target genes such as pyruvate dehydrogenase kinase 1 (PDK1) was increased in PHD2 deficient macrophages compared to wt cells under normoxic conditions. PDK1 is an important mediator of the metabolic adaptation during hypoxia and stimulates the shift to anaerobic glycolysis. Increased expression of PDK1 resulted in metabolic reprogramming in PHD2 deficient macrophages which was shown by increased lactate levels and decreased ATP levels. Compared to wildtype macrophages, PHD2 deficient macrophages demonstrated decreased oxygen consumption rates and increased glycolysis. The observed glycolytic phenotype was associated with an impaired migration and phagocytosis. The glycolytic phenotype and the defect in migration and phagocytosis were partially reversed by pharmacological inhibition of PDK using dichloroacetate (DCA). In summary, my data suggest that PHD2 plays an important role in macrophage function by modulating cellular metabolism through PDK1. 


\section{Introduction}

\subsection{Hypoxia}

Oxygen availability is a prerequisite for proper metabolism and function of all complex multicellular organisms (reviewed in (Carreau et al., 2011)). Consequently, optimal oxygen delivery to tissues and cells is crucial. As diffusion is not sufficient to provide enough oxygen to meet the metabolic needs of complex multicellular organisms, several strategies have been evolved to augment oxygen uptake and transport to the tissue. In mammals, oxygen is absorbed by the lungs and transported via the cardiovascular system.

To distinguish between different concentrations of oxygen in cell culture, the following terms are commonly used: Normoxia is defined as the oxygen partial pressure $\left(\mathrm{pO}_{2}\right)$ as measured at sea level, thus $19.95 \%, 150 \mathrm{mmHg}$ or $20.3 \mathrm{kPa}$. Most cell culture experiments are performed at this condition. Within the body, the normal $\mathrm{pO}_{2}$ is much lower and can vary a lot between different tissues, e.g. ranging from a $\mathrm{pO}_{2}$ of $150 \mathrm{mmHg}$ in the upper airways to $5 \mathrm{mmHg}$ in the retina (reviewed in (Taylor, 2008)). The normal $\mathrm{pO}_{2}$ of a tissue is defined as tissue normoxia (reviewed in (Carreau et al., 2011). Hypoxia takes place when the $\mathrm{pO}_{2}$ is lower than the normoxic concentration and occurs when the oxygen demand exceeds the oxygen availability.

Several adaptive mechanisms have developed to ensure adequate oxygen supply under hypoxic conditions (reviewed in (Taylor, 2008)). Examples of adaptive mechanisms are increase in the respiratory rate, in erythropoiesis and in angiogenesis. Furthermore, the metabolism can switch from aerobic to an anaerobic metabolism to use the remaining oxygen in the most efficient manner.

Hypoxia can occur under physiological and non-physiological conditions. During exercise, the oxygen demand of the working muscle increases (reviewed in (Garvey et al., 2012)). If the cardiovascular system cannot transport enough oxygen to keep up with this demand, hypoxia occurs. Hypoxia also occurs at high altitude because of the reduction of atmospheric $\mathrm{pO}_{2}$ (reviewed in (Wilkins et al., 2015). For example on top of the Mount Everest (approximately $8400 \mathrm{~m}$ ), the pressure of the inspired oxygen decreases from approximately $150 \mathrm{mmHg}$ at sea level to $43 \mathrm{mmHg}$. Furthermore, hypoxia is essential for embryonic development as much of the mammalian embryogenesis occurs at low oxygen concentrations (Chen et al., 1999; Dunwoodie, 2009; Lee et al., 2001). Studies using the hypoxia marker pimonidazole showed 
that hypoxia occurs within the embryo at different regions and at different times during organ development (Lee et al., 2001). These hypoxic regions were shown to be necessary for the development of fetal vascularization of the placenta (Dunwoodie, 2009). Pathophysiological conditions of hypoxia include progressive air flow limitation, e.g. chronic obstructive pulmonary disease (Kent et al., 2011). Furthermore it also occurs during ischemia, such as after myocardial infarction and often in tumors (reviewed in (Semenza, 2014; Vaupel and Mayer, 2007).

Therefore, a tight regulation of the oxygen availability is necessary. The key regulators of the hypoxic response at the cellular level are hypoxia inducible factors (HIF) (reviewed in (Dengler et al., 2014)).

\subsection{Macrophages}

Macrophages were originally identified by Ilya Metchnikoff more than 100 years ago, and his description of phagocytosis was awarded with the Nobel Prize for Medicine in 1908 (reviewed in (Gordon, 2016)).

Macrophages are essential innate immune cells and provide an important front line in the defense against pathogens (reviewed in (Sica and Mantovani, 2012)). Furthermore, they are also involved in homeostatic processes, such as tissue remodeling during embryogenesis and wound healing (Camp and Martin, 1996; Goren et al., 2009; Mirza et al., 2009). Macrophages are key players in the mediation of the inflammatory reaction and host defense and have an exceptional diversity and plasticity (reviewed in (Gordon and Martinez, 2010; Sica and Mantovani, 2012)). Peripheral-blood mononuclear cells (PBMC) are continuously released into the blood stream where they circulate for one to two days before entering the tissue to differentiate into macrophages (reviewed in (Mosser and Edwards, 2008). PBMCs are derived from myeloid progenitor cells in the bone marrow. These myeloid progenitor cells are also precursor cells for granulocytes and in part dendritic cells (reviewed in (Hume, 2015). The differentiation into macrophages or dendritic cells is dependent on the cytokine environment and on the activation of pattern recognition receptors (reviewed in (Geissmann et al., 2010)).

Mononuclear phagocytes react to environmental stimuli, such as microbial products, cell debris and activated lymphocytes with distinct polarization (reviewed in (Hume, 2015; Mosser and Edwards, 2008; Sica and Mantovani, 2012). They contain receptors that enable 
them to sense a broad range of signals and change their phenotype accordantly. Initially, macrophages polarization states were subdivided in M1 and M2 polarization of macrophages. In this subdivision, M1-polarized macrophages (or classically activated macrophages) are stimulated by Toll-like receptor ligands, interferon- $\gamma$ (IFN $\gamma$ ) and tumour necrosis factor $\alpha$ (TNF $\alpha$ ) (reviewed in (Biswas and Mantovani, 2012; Sica and Mantovani, 2012). Recognition of bacterial compounds, e.g. liposaccharide (LPS) can also result in M1 polarization of macrophages. M1 macrophages are described to be pro-inflammatory and tissue destructive. These macrophages are characterised by the expression of pro-inflammatory cytokines (such as interleukin-1 $\beta$, interleukin-6 (IL-6), interleukin-12 (IL-12), interleukin-23 (IL-23) and TNF $\alpha$ ), inducible nitric oxidase (iNOS), promotion of Th1 responses and have an important role in the clearance of pathogens.

On the other hand, M2-polarized macrophages (or alternative activated macrophages) are induced by interleukin-4/interleukin-13 (IL-4/IL-13). These macrophages are characterised as anti-inflammatory and are described to be involved in tissue remodeling as well as in angiogenesis. Furthermore, macrophages of this phenotype are involved in the clearance of parasites. Additional cellular markers for this polarization state are arginase-1, found in inflammatory zone 1 (Fizz 1) and Ym1 (Raes et al., 2002). Stimulation with IL10 and immune complexes can also induce a M2-like phenotype which is immune-regulatory and involved in pro-tumoral functions (reviewed in (Biswas and Mantovani, 2012)). These subdivisions, however, do not reflect the heterogeneity of macrophage polarization. Today, macrophage polarization states are rather described as a broad spectrum ranging from M1 to M2 with every intermediate state being independently defined depending on the nature of the polarization stimulus (reviewed in (Hume, 2015; Mosser and Edwards, 2008).

\subsubsection{Macrophages and hypoxia}

Ischemic and inflamed tissues are characterised by a scarcity of oxygen. The ability of the affected tissue to survive the reduction in oxygen and energy supply as well as the growth of new blood vessels is important for the outcome of an ischemic disease (Semenza, 2010). Migration into and accumulation of macrophages inside the damaged tissues is critical for these tissue remodeling processes. Azzawi et al. showed that macrophages accumulate in the cardiac tissue after myocardial infarction with macrophage accumulation peaking at day 5-7 (Azzawi et al., 2005). Cell migration is significantly affected by tissue oxygenation, which is 
severely impaired in ischemia. Approximately $95 \%$ of the myeloid cells migrate to the inflamed region rather than being resident at this tissue (reviewed in (Cramer et al., 2003; Lewis et al., 1999)). Therefore, myeloid cells need to be recruited to the site of inflammation against the oxygen gradient.

In hypoxic conditions, macrophages undergo a metabolic reprogramming to cope with the low oxygen availability and to be able to meet their energy requirements (reviewed in (Riboldi et al., 2013)). This is achieved by the induction of several genes by HIF. Hypoxic conditions have been reported to affect myeloid cell function in vitro regarding phagocytic capacity, cell surface marker expression, adhesion, migration and cell survival (reviewed in (Cramer et al., 2003; Riboldi et al., 2013)). It has been shown that macrophages accumulate in hypoxic regions, such as the ischemic myocardium, stabilize HIF and induce HIF-mediated gene expression (Burke et al., 2003; Jurgensen et al., 2004).

\subsection{The Hypoxia-Inducible Factor (HIF)}

In hypoxic conditions, myeloid cells and other cell types adapt to the environmental challenge via the induction of a specific gene expression program which is mediated by the hypoxia inducible factors (HIF). To date, HIFs are described to exist in all metazoan species (Loenarz et al., 2011). HIF-regulated gene expression controls the transcription of many genes involved in the systemic and cellular response to hypoxia (reviewed in (Liu et al., 2012; Semenza, 2004)). This leads to the promotion of angiogenesis and erythropoiesis as well as to the expression of genes involved in the metabolic adaption to hypoxic conditions (Bruick, 2000; Fukuda et al., 2007; Papandreou et al., 2006; Zhang et al., 2008).

\subsubsection{Structure of HIF}

HIF transcription factors are heterodimers consisting of one of the $\alpha$-subunits HIF-1 $\alpha$, HIF- $2 \alpha$ or HIF-3 $\alpha$ and a $\beta$-subunit (also called aryl hydrocarbon receptor nuclear translocator $(A R N T))$ (reviewed in (Dengler et al., 2014)). The two subunits are members of the Per-ArntSim (PAS) family containing a basic helix-loop-helix domain (bHLH) (Wang et al., 1995). The heterodimer binds to a specific DNA motif, the Hypoxia Responsive Elements (HREs). This binding then induces transcriptional activation of the target gene (reviewed in (Dengler et al., 2014)). The HIF-1 $\alpha$ subunit contains two transactivation domains (TAD) that are 
located at the N-terminus (N-TAD, aa 531-575) and at the C-terminus (C-TAD, aa 786-826) (Fig.1). The C-TAD can be bound by co-activators like CBP/p300 to activate transcription (Jiang et al., 1997; Kallio et al., 1998; Pugh et al., 1997). Furthermore, the HIF- $\alpha$ subunit contains an oxygen-dependent degradation domain (ODD) (Pugh et al., 1997). The ODD contains two conserved proline residues (P402/P564). These proline residues can be hydroxylated by the prolyl-4-hydroxylase domain enzymes (PHDs) (Ivan et al., 2002; Jaakkola et al., 2001). Additionally, the asparagine residue 803 (Asn803) in the C-TAD can be hydroxylated by factor inhibiting HIF (FIH) (Lando et al., 2002; Mahon et al., 2001).

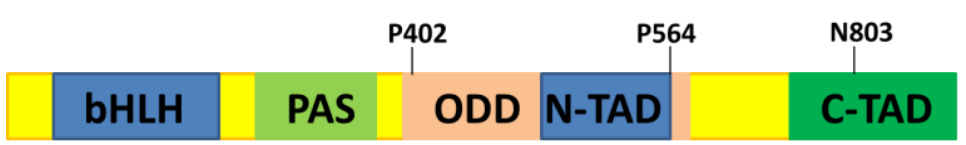

Figure 1: Domain structure of HIF-1 $\alpha$. The bHLH and the PAS domain are needed for DNA binding and dimerization, respectively. There are two transactivation domains present in the HIF-1 $\alpha$ protein: The N-TAD which resides in the oxygendependent degradation domain (ODD) and the C-TAD located at the C-terminal end. The ODD can be hydroxylated at two proline residues (P402, P564) by hydroxylating enzymes which leads to the oxygen-dependent degradation of HIF-1 $\alpha$. The C-TAD contains an asparagine residue (N803), which can be hydroxylated by FIH-1 leading to altered transcriptional activity.

The first discovered isoform was HIF-1 $\alpha$. It was found by analyzing proteins that bind to the HRE of the erythropoietin gene (EPO) (Semenza and Wang, 1992). The other two $\alpha$-subunits were identified using gene homology searches and by analyzing protein interaction partners of HIF-1 $\beta$ (reviewed in (Dengler et al., 2014)). HIF-1 $\alpha$ and HIF-2 $\alpha$ have a high similarity in bHLH and PAS domains and both bind to HREs to induce the transcription. HIF- $1 \alpha^{-/-}$mice die at embryonic day (E) 9.5 due to severe defects in vascularization, impaired neural folding and due to the reduction of somites (Iyer et al., 1998; Ryan et al., 1998). HIF-2/- mice also die in the embryo stage (Peng et al., 2000; Tian et al., 1998). Depending on the mouse model used this was due to brachycardia or vascular disorganization. HIF-1 $\alpha$ and HIF- $2 \alpha$ are known to have different roles in the adaption of cells to hypoxic conditions: HIF-1 initiates the response to hypoxia, whereas HIF-2 is important for the adaptation to long-term hypoxia (Koh et al., 2011). The HIF-3 $\alpha$ subunit is less similar compared to HIF-1 $\alpha$ and HIF-2 $\alpha$. There are at least six splice variants of HIF-3 $\alpha$ which have variable oxygen sensitivity and differences in their regulatory functions of transcription (reviewed in (Dengler et al., 2014). When expressed as an alternative splice variant called HIF3- $\alpha 4$ which is highly similar to the murine Inhibitory PAS domain protein 1, it negatively regulates the HIF-1 $\alpha$ response (Dengler et al., 2014; 
Makino et al., 2007; Maynard et al., 2005). This variant lacks the TAD and therefore does not activate target gene expression, but rather acts as a HIF-1 $\alpha$ inhibitor.

\subsubsection{Regulation of HIFs by Prolyl-4-Hydroxylase Domain enzymes (PHD) and the Factor Inhibiting HIF (FIH)}

The HIF-1 $\beta$ subunit is stably expressed and its mRNA and protein levels are not affected by changes in oxygen availability. On the contrary, the regulation of HIF- $\alpha$ occurs at the level of protein stability through the action of hydroxylating enzymes (Fig.2). HIF-1 $\alpha$ is hydroxylated in the ODD domain by the prolyl-4 hydroxylase domain enzymes (PHDs) (Bruick and McKnight, 2001; Epstein et al., 2001). The hydroxylation by the PHDs targets HIF-1 $\alpha$ for proteasomal degradation which is mediated by the pVHL-E3-ubiquitin-ligase complex (Huang et al., 1998; Maxwell et al., 1999; Ohh et al., 2000; Pause et al., 1999; Tanimoto et al., 2000). These processes result in a half-life time of the HIF- $\alpha$ subunit of approximately five minutes in normoxic conditions (Moroz et al., 2009). The $\mathrm{K}_{\mathrm{m}}$-values of the PHDs for oxygen are between $230-250 \mu \mathrm{M}$ which is only slightly above the values for the atmospheric $\mathrm{pO}_{2}$ at sea level and therefore also above the oxygen concentrations observed in physiological conditions in many tissues (Hirsila et al., 2003). This causes the PHDs to have a relatively low affinity to oxygen. This feature makes them suitable oxygen sensors in mammals. At low oxygen concentrations the hydroxylation of the HIF- $\alpha$ subunits ceases and HIF- $\alpha$ is stabilized.

Stabilization of the HIF-1 $\alpha$ subunit is not sufficient to induce HIF target gene expression (reviewed in (Ke and Costa, 2006)). The transcriptional activity of HIF-1 is inhibited by the interaction with the factor inhibiting HIF-1 (FIH-1) (Hewitson et al., 2002; Lando et al., 2002; Mahon et al., 2001). FIH-1 hydroxylates a conserved asparagine residue (Asn803) in the CTAD of the HIF-1 $\alpha$ subunit in an oxygen sensitive manner. Hydroxylation of this residue blocks the interaction of HIF-1 $\alpha$ with the transcription adaptors cAMP response elementbinding protein (CREB)-binding protein (CBP) and p300 ((Arany et al., 1996) and reviewed in (Dengler et al., 2014)). Inhibition of CBP/p300 recruitment by FIH-1 thereby inhibits the execution of HIF-1 transcriptional activity. 


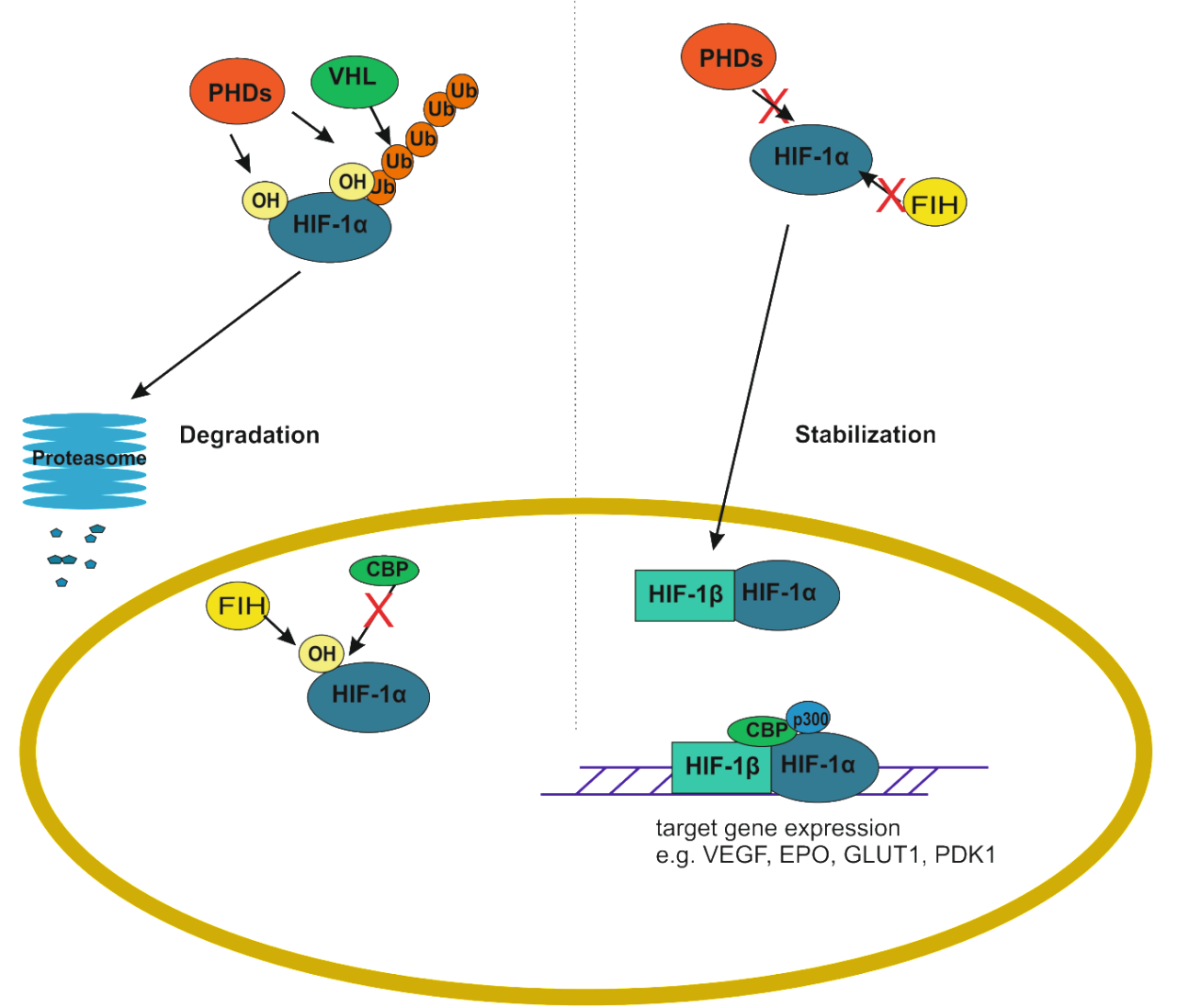

Figure 2: Summary of the regulation of HIF-1 $\alpha$ protein levels. In normoxia, hypoxia-inducible factor $1-\alpha(\mathrm{HIF}-1 \alpha)$ is located in the cytosol and hydroxylated by prolyl-4 hydroxylase domain enzymes (PHDs). This hydroxylation leads to the ubiquitination of HIF-1 $\alpha$ by von Hippel-Lindau tumor suppressor protein (pVHL) and subsequently to the proteasomal degradation of the HIF-1 $\alpha$ subunit. HIF- $1 \alpha$ is also hydroxylated by factor inhibiting HIF (FIH) thereby blocking the interaction of HIF-1 $\alpha$ with cAMP response element-binding protein (CREB) and p300 (CBP/p300). In hypoxia, the hydroxylation of HIF- $1 \alpha$ ceases and HIF- $1 \alpha$ translocates to the nucleus and dimerizes with hypoxia-inducible factor $1-\beta$ (HIF-1 $\beta$ ). After binding to $\mathrm{CBP} / 300$, the transcription of HIF target genes, such as vascular endothelial growth factor (VEGF), erythropoietin (EPO), Glucose transporter 1 (GLUT1) and pyruvate dehydrogenase kinase 1 (PDK1) is induced.

\subsubsection{HIF target genes}

HIFs are the principal transcriptional regulators of oxygen-dependent gene expression with at least 100 known target genes (reviewed in (Liu et al., 2012)). The target genes are involved in the regulation of angiogenesis, cell migration and metabolic adaptation to scarce oxygen availability. Induction of these processes leads to a decrease in oxygen consumption of the cells and to an increase of oxygen delivery to the cells in order to maintain an adequate oxygen supply to the tissue (Fukuda et al., 2007; Papandreou et al., 2006). The oxygen 
transport is mediated by HIF-1 via the expression of EPO (reviewed in (Li et al., 2004).). EPO stimulates erythroid progenitor cells to increase erythropoiesis. Furthermore, it is also involved in the proliferation and differentiation of other cell types such as endothelial cells, vascular smooth muscle cells, neurons and neuronal progenitor cells. HIF-1 also induces the expression of inducible nitric oxide synthase (iNOS) (Melillo et al., 1995). This increases vasodilatation and hence facilitates oxygen delivery. A very prominent HIF-target gene is the vascular endothelial growth factor (VEGF) which stimulates angiogenesis and vascularization (Forsythe et al., 1996; Liu et al., 1995). All these adaptions increase the oxygen transport to the tissue and thereby improving the oxygen availability in the tissue.

\subsubsection{Metabolic adaptations to hypoxia}

In order to meet energy demands in hypoxic conditions, the cellular energy metabolism needs to be adapted to the decrease in oxygen availability. Therefore, the metabolism is shifted from aerobic to anaerobic glycolysis. In normoxia, glucose enters the cell and is metabolized in several steps to pyruvate which enters the mitochondria and is there converted to acetyl-CoA by pyruvate dehydrogenase (PDH) (reviewed in (Dashty, 2013; Taylor, 2008)) (Fig.3). Acetyl CoA is used in the tricarboxylic acid (TCA) cycle to produce ATP and to reduce NAD+ to $\mathrm{NADH}$. NADH, in turn is then fed into the respiratory chain. The respiratory chain consists of four enzymes: NADH hydrogenase (complex I), succinate-ubiquinone oxidoreductase (complex II), ubiquinol cytochrome c oxidoreductase (complex III), cytochrome c oxidase (COX, complex IV). The first four enzymes (complex I-IV) and the two mobile electron carriers ubiquinone and cytochrome $\mathrm{c}$ form the respiratory chain. The respiratory chain couples electron transport with pumping of protons to build up the electrochemical gradient needed by the ATP synthase to produce ATP.

HIF-1 mediates the shift towards anaerobic glycolysis by the upregulation of several target genes, such as the Glucose transporter 1 (GLUT1) (Ebert et al., 1995; Hayashi et al., 2004) to increase glucose uptake and the expression of enzymes which are involved in the glycolytic pathway such as the phosphofructokinase 1 (PFK1) and lactate dehydrogenase (Firth et al., 1995; Obach et al., 2004; Semenza et al., 1996; Semenza et al., 1994). Furthermore, HIF-1 represses oxygen consumption and attenuates mitochondrial respiration by the induction of PDK1 (Kim et al., 2006; Papandreou et al., 2006). Phosphorylation by PDK1 inhibits the action of PDH. It was first shown by Linn et. al. that mitochondrial function could be 
regulated by phosphorylation of PDH and that this is mediated by PDKs (Linn et al., 1969). $\mathrm{PDH}$ is one part of a mitochondrial multi-enzyme complex that catalyzes the oxidative decarboxylation of pyruvate to acetyl-CoA (reviewed in (Sugden and Holness, 2003)). This reduces the flux of acetyl CoA through the mitochondrial TCA cycle and the electron transport chain and reduces the mitochondrial oxygen consumption likewise.

Another mechanism which increases respiratory efficiency in hypoxic cells is the regulation of cytochrome c oxidase (COX) activity (Fukuda et al., 2007). During hypoxia, HIF-1 $\alpha$ induces the transcription of the genes encoding the COX4-2 subunit and the Lon protease. This mitochondrial protease degrades the subunit COX4-1 which is predominantly expressed in normoxic conditions. The cytochrome c subunit switch (COX4-1 to COX4-2) increases the efficiency of complex IV of the mitochondrial electron transport chain regarding quantity of ATP and reactive oxygen species (ROS) produced. By doing so, mitochondrial oxygen consumption is reduced. If the metabolic adaptation fails, HIF-1 induces the expression of BCL/adenovirus E1B $19 \mathrm{kDa}$ interacting protein 3 (BNIP3) to prevent increased ROS levels and cell death in hypoxic cells (Bruick, 2000; Zhang et al., 2008). BNIP3 competes for binding to Bcl-2, releasing Beclin-1 to stimulate mitochondrial autophagy (Maiuri et al., 2007).

Altogether, these changes reduce the flux through the mitochondrial TCA cycle and the electron transport chain. This decreases oxygen consumption and reduces the accumulation of ROS likewise. 


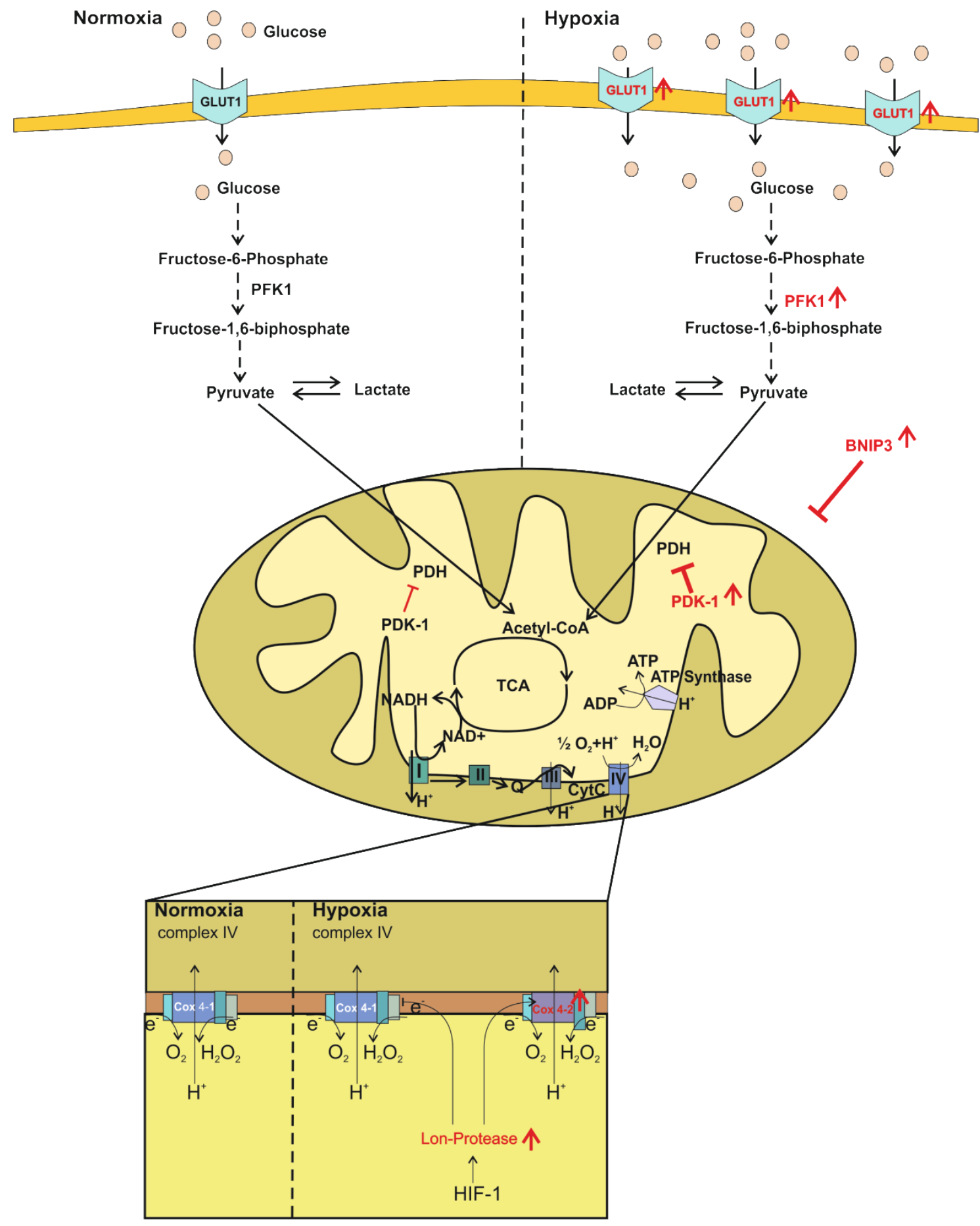

Figure 3: Induction of metabolic adaptation by hypoxia-inducible factor (HIF). In normoxia, glucose is converted to pyruvate which enters the mitochondria and is there metabolized to acetyl-CoA by pyruvate dehydrogenase (PDH). Acetyl CoA then enters the tricarboxylic acid cycle (TCA). In hypoxia, HIF-1 transcriptionally upregulates the expression of glucose transporter 1 (Glut1) and phosphofructokinase 1 (PFK1). HIF inhibits pyruvate dehydrogenase (PDH) by transcriptionally increasing the expression of PDH kinase (PDK), which phosphorylates and inactivates PDH. HIF-1 also leads to changes in the respiratory chain. In normoxia, Cox 4-1 is the predominant isoform of Cox4. During hypoxia, HIF-1 $\alpha$ activates Lon Protease to degrade Cox 4-1 and also upregulates Cox 4-2 to replace Cox 4-1. 


\subsection{Prolyl-4-hydroxylase domain enzymes (PHDs)}

PHDs are evolutionarily conserved oxygen sensors in metazoans. The PHDs belong to the iron- and $\alpha$-ketoglutarate-dependent dioxygenase superfamily and were discovered after confirming that oxygen-sensitive enzymes modify the ODD of the HIF-1 $\alpha$ subunit (Huang et al., 1998; Jaakkola et al., 2001).

Worms and flies have only one PHD whereas there exist at least three members of the PHD protein family in mammals: PHD1 (also EGLN2), PHD2 (also called EGLN1) and PHD3 (also called EGLN3) (Bruick and McKnight, 2001; Epstein et al., 2001; Kaelin and Ratcliffe, 2008). Recently, a fourth member of the PHD family, the prolyl 4-hydroxylase transmembrane (TM-HIF-P4H), was identified, but its function is not fully characterised yet (Koivunen et al., 2007; Laitala et al., 2012). In general, all three PHD isoforms are ubiquitously expressed, but expression levels vary between the tissues (Appelhoff et al., 2004; Cioffi et al., 2003; Lieb et al., 2002). PHD2 is constitutively expressed in all tissues, whereas PHD1 is highly expressed in the testis and hormone responsive tissues and PHD3 is predominantly expressed in heart, skeletal muscles and in the placenta. Studies using PHD proteins fused to the Green Fluorescent Protein (GFP) showed that PHD1 is mainly localized in the nucleus, PHD2 is predominantly localized in the cytoplasm and PHD3 is distributed homogenously in both compartments (Metzen et al., 2003; Metzen et al., 2005)

\subsubsection{Role of PHDs}

Oxygen-dependent degradation after PHD-mediated hydroxylation is the primary pathway of regulating the activity of HIF transcription factors (Epstein et al., 2001). In normoxic conditions, the two critical prolines of the ODD (Pro564 and Pro402) are hydroxylated by PHDs in the presence of oxygen targeting HIF $\alpha$ for proteasomal degradation. Mutation in either proline is sufficient for HIF $\alpha$-stabilization in normoxic conditions. Studies reported that the two proline residues of HIF $\alpha$ are differently regulated by the PHDs (Chan et al., 2005; Epstein et al., 2001; Huang et al., 2002). Using hydroxylation specific antibodies, it was shown that in normoxic conditions Pro564 is hydroxylated prior to Pro402 and that a mutation in Pro564 also reduces the hydroxylation of Pro402. Furthermore, Pro402 showed an oxygendependent decrease in hydroxylation at high oxygen tensions indicating that hydroxylation of Pro402 is highly responsive to changes in oxygen and therefore has a more important role in HIF regulation in hypoxic conditions (Chan et al., 2005). In addition to that, it was reported 
that PHD2 has higher affinity for Pro402 than for Pro564 (Huang et al., 2002). PHD2 and 3 have a similar catalytic activity towards a synthetic hydroxylated HIF-1 $\alpha$ peptide, whilst the catalytic activity of PHD1 is lower (Tuckerman et al., 2004). Studies using RNA-interference revealed that silencing of PHD2 is sufficient to stabilize and activate HIF-1 $\alpha$ in normoxia, whilst silencing of either PHD1 or PHD3 alone has no effect on HIF-1 $\alpha$ stability in normoxia (Berra et al., 2003). This suggests that PHD2 is the critical oxygen sensor in normoxia. PHD2 and PHD3 show an upregulation in hypoxia whereas PHD1 is expressed constitutively (Epstein et al., 2001; Metzen et al., 2003). This suggests a negative feedback loop in the regulation of the HIF-mediated response (Appelhoff et al., 2004; del Peso et al., 2003; Pescador et al., 2005).

\subsubsection{PHD hydroxylation reaction}

PHD enzymes catalyze a hydroxylation reaction (reviewed in (Berra et al., 2006; McDonough et al., 2006)). For this, a single non-heme bound $\mathrm{Fe}^{2+}$ is needed for the activation of $\mathrm{O}_{2}$ and as a template for the correct binding of the reactants. One oxygen atom is added to peptidyl proline resulting in the formation of hydroxyproline. The remaining oxygen atom is used in a coupled decarboxylation reaction that results in $\alpha$-ketoglutarate to be converted to succinate. In this reaction also $\mathrm{CO}_{2}$ and $\mathrm{Fe}^{3+}$ are produced. The hydroxylation reaction is furthermore dependent on the presence of ascorbate that is required for the recycling of $\mathrm{Fe}^{3+}$ to $\mathrm{Fe}^{2+}$, which can then be used in the next catalytic cycle (Counts et al., 1978; Nietfeld and Kemp, 1981; Schofield and Ratcliffe, 2004; Smirnova et al., 2012). Depletion of ascorbate was found to inhibit PHD function and to stabilize HIF-1 $\alpha$ in human cancer cells (Knowles et al., 2003).

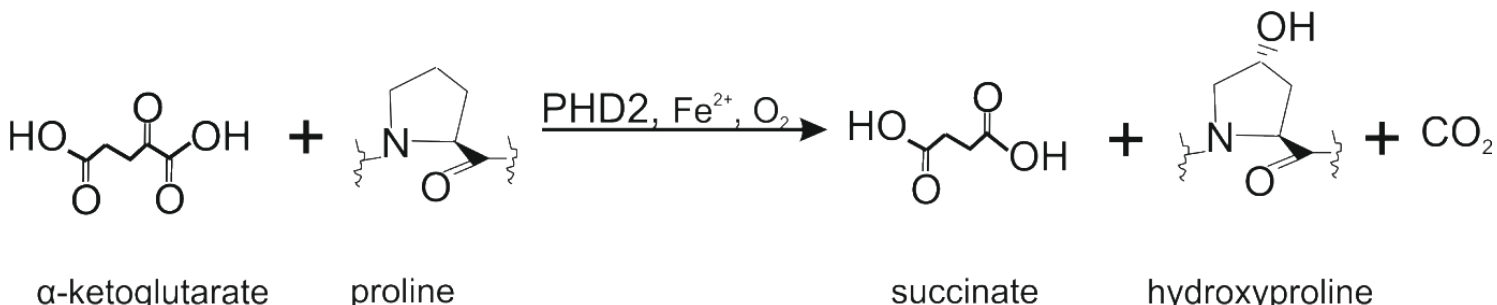

Figure 4: Hydroxylation reaction of prolyl-4-hydroxylases (PHD). The PHDs utilize $\mathrm{O}_{2}$ and $\alpha$-ketoglutarate as substrates and $\mathrm{Fe}^{2+}$ as co-factor. In the hydroxylation reaction, 4-hydroxyproline and succinate are formed. 


\subsubsection{Biological function of PHDs}

Several genetically modified mouse models have been developed to study the role of PHDs in vivo.

\subsubsection{PHD1 genetically modified mouse models}

PHD1 is reported to have a role in mitochondrial metabolism (Aragones et al., 2008) and ROS production (Schneider et al., 2010). PHD1 $^{-/}$mice showed no obvious phenotype under non-stressed conditions except for a reduced mitochondrial oxygen consumption rate (OCR) in skeletal muscle cells (Aragones et al., 2008). This metabolic reprogramming decreased muscle performance in healthy tissue. On the other hand, knockout of PHD1 was shown to attenuate myocardial ischemia/reperfusion injury in the isolated heart by enhancing HIF-1 $\alpha$ signaling (Adluri et al., 2011). Furthermore, $\mathrm{PHD}^{-/-}$mice were protected against colitis (Tambuwala et al., 2010). This was associated with an improved epithelial barrier function because of decreased epithelial apoptosis. Reduced apoptosis upon PHD1-depletion was also described in a model of hyperoxia-induced vascular obliteration in the retina (Huang et al., 2011). Liver regeneration was significantly improved in PHD $1^{-/-}$mice due to an enhanced proliferation of hepatocytes indicating a role of PHD1 on cell cycle progression (Mollenhauer et al., 2012). Until now, no myeloid specific PHD1 knockout mouse model has been generated and the role of PHD1 in macrophage function has not been described.

\subsubsection{PHD3 genetically modified mouse models}

Mice with a general knockout of PHD3 showed a reduction of neuronal apoptosis, reduced function of the sympathoadrenal system and systemic hypotension (Bishop et al., 2008). Additionally, depletion of PHD3 was described to be protective against cardiomyocyte apoptosis after ischemia/reperfusion injury (Xie et al., 2015). Furthermore, PHD3 was described to have a function in the hypoxic regulation of the innate immune response (Thompson et al., 2013). PHD3 ${ }^{-/-}$neutrophils showed a decreased hypoxic survival compared with wild type (wt) cells (Walmsley et al., 2011). Analysis of PHD3 ${ }^{-/-}$bone marrow derived macrophages (BMDM) showed a role of PHD3 in the regulation of macrophage function. One study showed a role of PHD3 in macrophage differentiation five days after differentiation which was accompanied with a change to a pro-inflammatory phenotype of the macrophages 
(Kiss et al., 2012). Another study could also observe an effect of PHD deficiency in the differentiation of BMDMs until day 5 (Swain et al., 2013). Anyhow, eight days after the onset of the differentiation, no differentiation difference was observed and also M1 and M2 polarization was unchanged between $\mathrm{PHD}^{-/-}$and wt BMDMs. Under stress conditions, PHD3-deficient BMDMs displayed a reduction in TUNEL positive cells and had decreased apoptosis.

\subsubsection{PHD2 genetically modified mouse models}

Previous studies showed that PHD2 ${ }^{-/-}$mouse embryos die between day E 12.5 and E14.5 due to severe vascular defects in placenta and heart during embryogenesis (Minamishima et al., 2008; Takeda et al., 2006). PHD2 is essential for HIF-1 $\alpha$-mediated angiogenesis since postnatal somatic deletion of PHD2 in mice resulted in increased density and size of blood vessels, whereas loss of PHD1 or PHD3 did not have comparable effect (Takeda et al., 2007). Additionally, somatic PHD2 inactivation was found to lead to profound polycythemia (Minamishima et al., 2008).

Work performed in various inducible mouse models indicate that the effect of PHD2 depends very much on the targeted cell type. Depending on the cell type in which PHD2 has been manipulated, i.e. tumor cells or endothelial cells, PHD2 was found to be a potent mediator of tumor angiogenesis pathways or in vessel normalization in tumors (Chan et al., 2009; Mazzone et al., 2009). Haplodeficiency of PHD2 had no effect on the density of tumor vessels and lumen size, but changed the phenotype of endothelial tip cells to a more quiescent cell type. Additionally, it enhanced vessel maturation leading to an improved tumor perfusion and less tumor cell invasion (Mazzone et al., 2009). On the other hand, interleukin 8 (IL-8) and angiogenin are produced by PHD2 deficient tumor cells and contribute to bone marrowderived cell mobilization and angiogenesis which increases tumor growth. These two factors were found to be mediated by PHD2 in tumor cells in a HIF-independent manner providing evidence for a critical role of PHD2 per se in controlling tumor growth (Chan et al., 2009). Furthermore, conditional ablation of PHD2 in myeloid cells and T-lymphocytes reduced tumor volume (Mamlouk et al., 2014). This effect was mediated by the overall downregulation of both anti-inflammatory cytokines and pro-inflammatory cytokines. In addition, Kalucka et al. found that only PHD2-deficiency in keratinocytes, but not in myeloid 
cells led to a faster wound closure induced by an enhanced migratory capacity (Kalucka et al., 2013).

Invading macrophages are major players for tissue regeneration and angiogenesis in ischemic tissues. PHD2 ${ }^{+/-}$-heterozygous mice had more M2-like polarized macrophages which are important for the preservation of tissue perfusion and protection (Takeda et al.). Accordantly, $\mathrm{PHD}^{+/-}$mice exhibited increased viability of the ischemic tissue in a model of hindlimb ischaemia. Furthermore PHD$^{+/-}$mice were also protected from myocardial ischemia. Desmin-stainings showed less cardiomyocyte death in $\mathrm{PHD}^{+/-}$mice compared to wt controls $24 \mathrm{~h}$ after coronary artery ligation. Most interestingly, these findings were obtained with $\mathrm{PHD}^{+/-}$mice and LysMCrexPHD2 $2^{\mathrm{fl} /+}$ mice and could not be recapitulated in LysM Cre PHD2 fl/fl mice indicating a dose dependent effect (Ikeda et al., 2013; Takeda et al., 2011). In other studies, PHD2 deficient macrophages isolated from LysMCre ${ }^{+/} \mathrm{xPHD}^{f l f l}$ mice were found to have decreased markers of M1-polarization without a clear polarization to M2 macrophages which is in contrast to the observations above (Ikeda et al., 2013). PHD2 deficient macrophages displayed a decreased migratory capacity toward monocyte chemoattractant protein-1 (MCP-1) in a transwell migration assay. In a model of hypertension induced by LNAME and angiotensin-treatment, PHD2-deletion in macrophages led to less macrophage infiltration in the aorta, attenuated cardiovascular remodeling and preserved heart function. 


\subsection{Aims of this thesis}

Macrophages are key players of the innate immune system and are responsible for the rapid recognition of invading pathogens. They play an important role in the mediation of the inflammatory reaction and display a notable plasticity. Ischemic and inflamed tissues are characterised by scarce oxygen availability. Macrophages accumulate inside these tissues and are involved in remodelling processes. Therefore, they have to migrate against the oxygen gradient. Like other cells, macrophages adapt to the resulting hypoxic conditions via inducing a HIF-mediated gene expression program. The oxygen dependent hydroxylation of the HIF- $\alpha$ subunit by the PHDs marks HIF- $\alpha$ for proteasomal degradation. Of the three PHDs, PHD2 has been described to be the main oxygen sensor in normoxia. Deficiency of PHD2 has been reported to have cell type-specific effects. However, the effect of PHD2 in macrophages is not fully understood. To analyze the impact of PHD2 on macrophage function in further depth, I used myeloid specific conditional PHD2 knockout mice $\left(\mathrm{LysMCre}^{+/-} \mathrm{x}\right.$ Phd2 ${ }^{\text {flox/flox }}$, PHD2 cKO) and stably transduced RAW cells expressing a PHD2 shRNA construct (shPHD2 RAW). I aimed to investigate how metabolism and function of macrophages are influenced by PHD2 deficiency. To this end, the following objectives were defined:

1. To analyse HIF-1 $\alpha$ stabilization in PHD2 cKO and shPHD2 RAW macrophages in normoxia.

2. To test if HIF-1 target genes are differentially expressed in PHD2 cKO and shPHD2 RAW macrophages.

3. To identify changes in macrophage function in PHD2 cKO and shPHD2 RAW macrophages.

4. To characterize metabolic alterations in PHD2 cKO and shPHD2 RAW macrophages.

5. To determine if the metabolic changes depend on the expression of the HIF target gene PDK1. 


\section{Material and Methods}

\subsection{Cell lines}

The cell lines used in this project are depicted in Table 1.

Table 1: Cell lines

\begin{tabular}{|l|l|l|l|}
\hline Cell line & Source & Distributor & Reference \\
\hline RAW264.7 & $\begin{array}{l}\text { Abelson murine leukemia virus } \\
\text { transformed, macrophage }\end{array}$ & $\begin{array}{l}\text { Kindly provided by } \\
\text { Dr. B. Wielockx, } \\
\text { Institute of Pathology, } \\
\text { TU Dresden GER }\end{array}$ & $\begin{array}{l}\text { (Raschke et } \\
\text { al., 1978) }\end{array}$ \\
\hline kDA-MB 231 & $\begin{array}{l}\text { kuman, Mammary } \\
\text { Gland/Breast; epithelial }\end{array}$ & $\begin{array}{l}\text { Dr. R. Wenger, } \\
\text { Institute of } \\
\text { Physiology, Zürich } \\
\text { CH }\end{array}$ & $\begin{array}{l}\text { (Young et } \\
\text { al., 1974) }\end{array}$ \\
\hline L929 & $\begin{array}{l}\text { ATCC, Wesel GER } \\
\text { \# CCL-1 }\end{array}$ & $\begin{array}{l}\text { (Earle et al., } \\
\text { tissue, fibroblast }\end{array}$ \\
\hline
\end{tabular}

\subsection{Consumable supplies}

The consumable supplies are listed in Table 2.

Table 2: Consumable supplies

\begin{tabular}{|l|l|}
\hline Consumables & Manufacturer \\
\hline Cell culture flasks & Greiner Bio-One, Kremsmünster AU \\
\hline Falcon tubes $15 \mathrm{ml}$ & Greiner Bio-One, Kremsmünster AU \\
\hline Falcon tubes $50 \mathrm{ml}$ & Greiner Bio-One, Kremsmünster AU \\
\hline Filter $(0.22 \mu \mathrm{m})$ & Roth, Karlsruhe GER \\
\hline Hybond ${ }^{\text {TM }}$ C nitrocellulose membrane & Amersham Biosciences, UK \\
\hline Original-Perfusor Syringe OPS $10 \mathrm{ml}$ & Braun, Melsungen GER \\
\hline Pasteur pipettes & Sarstedt, Nümbrecht GER \\
\hline PCR-plate $(96$ well) & Greiner Bio-One, Kremsmünster AU \\
\hline Pipette tips (w/o filters) & Sarstedt, Nümbrecht GER \\
\hline Reaction tubes $0.5 \mathrm{ml}$ & Sarstedt, Nümbrecht GER \\
\hline Reaction tubes $1.5 \mathrm{ml}$ & Sarstedt, Nümbrecht GER \\
\hline Reaction tubes $2.0 \mathrm{ml}$ & Sarstedt, Nümbrecht GER \\
\hline Serological pipettes $10 \mathrm{ml}$ & Sarstedt, Nümbrecht GER \\
\hline Serological pipettes $25 \mathrm{ml}$ & Sarstedt, Nümbrecht GER \\
\hline Serological pipettes $5 \mathrm{ml}$ & Sarstedt, Nümbrecht GER \\
\hline Tissue culture dish $10 \mathrm{~cm}$ & Greiner Bio-One, Kremsmünster AU \\
\hline Tissue culture dish $20 \mathrm{~cm}$ & Greiner Bio-One, Kremsmünster AU \\
\hline
\end{tabular}




\begin{tabular}{|l|l|}
\hline Tissue culture plates, 24 well & $\begin{array}{l}\text { Greiner Bio-One, Kremsmünster AU, Sarstedt, } \\
\text { Nümbrecht GER }\end{array}$ \\
\hline Tissue culture plates, 6 well & Greiner Bio-One, Kremsmünster AU \\
\hline Tissue culture plates, 96 well & Sarstedt, Nümbrecht GER \\
\hline Luminometer plates (cell culture) & Greiner Bio-One, Kremsmünster AU \\
\hline XF calibration plates & Seahorse Bioscience, North Billerica, USA \\
\hline XF cell plates & Seahorse Bioscience, North Billerica, USA \\
\hline X-ray films & GE Healthcare, Munich, GER \\
\hline
\end{tabular}

\subsection{Equipment}

The equipment used in this project is given in Table 3.

Table 3: Equipment

\begin{tabular}{|c|c|c|}
\hline Application & Instrument & Manufacturer \\
\hline \multirow[t]{3}{*}{ Centrifugation } & Centrifuge 5415R & Eppendorf, Hamburg, GER \\
\hline & Centrifuge 5810R & Eppendorf, Hamburg, GER \\
\hline & Micro Centrifuge & Carl Roth, Karlsruhe, GER \\
\hline Cell freezer & Nalgene freeze $-80^{\circ} \mathrm{C}$ & Nalgene, Rochester, USA \\
\hline Cleanbench & Hera Safe KS 12 & $\begin{array}{l}\text { Thermo Electron, } \\
\text { Langenselbold, GER }\end{array}$ \\
\hline Flow cytometer & $\begin{array}{l}\text { BD FACS Canto II } \\
\text { Flowcytometer }\end{array}$ & BD Biosciences, GER \\
\hline Fluorescence microscope & Axio Observer D1 & Zeiss, Oberkochen, GER \\
\hline Inverse light microscope & Motic $®$ AE30 & TED, Pella Inc, USA \\
\hline Heating block & $\begin{array}{l}\text { Block thermos stat } \\
\text { BT2000 }\end{array}$ & Kleinfeld Labortechnik, GER \\
\hline SDS-Page & $\begin{array}{l}\text { PerfectBlue Double gel } \\
\text { system }\end{array}$ & Peqlab, Erlangen, GER \\
\hline Shaker & Minitron & $\begin{array}{l}\text { INFORNS HAT AG, } \\
\text { Bottmingen, } \mathrm{CH}\end{array}$ \\
\hline Cell culture incubator & CB159 & Binder, Tuttlingen, GER \\
\hline Luminometer & Centro LB 960 & Berthold, Bad Wildbad, GER \\
\hline Magnetic stirrer & Ikamag®RH & IKA Labortech, Staufen, GER \\
\hline Microplate Reader & Model 680 & Bio-Rad, München, GER \\
\hline \multirow[t]{2}{*}{$\begin{array}{l}\text { Oxygen-controlled work } \\
\text { station }\end{array}$} & In $\mathrm{Vivo}_{2} 400$ & $\begin{array}{l}\text { Baker Ruskin Technologies, } \\
\text { Bridgeend, UK }\end{array}$ \\
\hline & Sci-tive & $\begin{array}{l}\text { Baker Ruskin Technologies, } \\
\text { Bridgeend, UK }\end{array}$ \\
\hline $\begin{array}{l}\text { Seahorse: Oxygen } \\
\text { consumption, Glycolysis }\end{array}$ & XF96 analyzer & $\begin{array}{l}\text { Seahorse Bioscience, North } \\
\text { Billerica, USA }\end{array}$ \\
\hline
\end{tabular}




\begin{tabular}{|l|l|l|}
\hline PCR cycler & Primus 96 Thermocycler & Peqlab, Erlangen, GER \\
\hline Real-Time PCR cycler & Mx3000P & Strategene, La Jolla, USA \\
\hline UV-transilluminator & InGenius & Syngene, Cambridge, UK \\
\hline Vortex & Vortex Genie 2 & $\begin{array}{l}\text { Scientific Industries, München, } \\
\text { GER }\end{array}$ \\
\hline Water bath & Störktronic W22 & Medigen, Martinsried, GER \\
\hline Western blot transfer & $\begin{array}{l}\text { PerfectBlue Semi-Dry } \\
\text { electroblotter }\end{array}$ & Peqlab, Erlangen, GER \\
\hline HPLC system & Shimadzu & Shimadzu, Kyoto, JP \\
\hline
\end{tabular}

\subsection{Software}

The software used in this project is given in Table 4 .

Table 4: Software

\begin{tabular}{|l|l|l|}
\hline Program & Application & Source \\
\hline CorelDraw & Image editor & Corel Corporation, Ottawa, USA \\
\hline Image J & Image analysis & Imagej.nih.gov \\
\hline Image J plugins & Manual tracking & $\begin{array}{l}\text { http://rsb.info.nih.gov/ij/plugins/manual- } \\
\text { tracking.html }\end{array}$ \\
\cline { 2 - 3 } & Chemotaxis & $\begin{array}{l}\text { http://ibidi.com/software/chemotaxis_and_ } \\
\text { migration_tool/ }\end{array}$ \\
\hline BLAST & Sequence alignment & Ncbi.nlm.nih.gov \\
\hline $\begin{array}{l}\text { GraphPad Prism } \\
\text { outlier calculator }\end{array}$ & Statistical analysis & GraphPad Software Inc, La Jolla, USA \\
\hline Endnote & Statistical analysis & GraphPad Software Inc, La Jolla, USA \\
\hline BD FACSDiva & References & Thomson Reuters, Philadelphia, USA \\
\hline Flowing Software 2 & FACS analysis & BD Biosciences, New Jersey, USA \\
\hline $\begin{array}{l}\text { Windows Office Excel } \\
\text { 2010 }\end{array}$ & Data analysis & $\begin{array}{l}\text { Perttu Terhu, Turku Centre of } \\
\text { Biotechnology, FI }\end{array}$ \\
\hline
\end{tabular}




\subsection{Chemicals}

The chemicals used in this project are given in Table 5.

Table 5: Chemicals

\begin{tabular}{|c|c|c|}
\hline Chemical & Order number & Manufacturer \\
\hline Accutase & P10-21100 & PAA, Pasching, AUS \\
\hline Acrylamide & 03029.1 & Carl Roth, Karlsruhe, GER \\
\hline Adenosine-triphosphate (ATP) & A2754 & Sigma, St. Louis, USA \\
\hline Ascorbate & A5960 & Sigma, St. Louis, USA \\
\hline Albumine Bovine Fraction V (BSA) & A1391 & Applichem, Darmstadt, GER \\
\hline$\beta$-mercaptoethanol & 4227.1 & Carl Roth, Karlsruhe, GER \\
\hline Bradford solution & $500-0006$ & $\begin{array}{l}\text { Bio-Rad Laboratories GmbH, } \\
\text { Munich, GER }\end{array}$ \\
\hline Bromphenol blue & A512.1 & Carl Roth, Karlsruhe, GER \\
\hline Chloroform & 3313.1 & Carl Roth, Karlsruhe, GER \\
\hline $\begin{array}{l}\text { Complete Mini EDTA-free Protease } \\
\text { Inhibitor }\end{array}$ & 04693124001 & Roche, Mannheim, GER \\
\hline Complete Mini PhosStop & 04906837001 & Roche, Mannheim, GER \\
\hline Coumaric acid & C-9008 & Sigma, St. Louis, USA \\
\hline DC kit & $500-0114$ & $\begin{array}{l}\text { Bio-Rad Laboratories GmbH, } \\
\text { Munich, GER }\end{array}$ \\
\hline DHE & D2926 & Sigma, St. Louis, USA \\
\hline DMSO & 7029.1 & Merck, Schwalbach/Ts, GER \\
\hline Dispase II & P10-032100 & PANTM biotech, Passau, GER \\
\hline EDTA & 8043.2 & Carl Roth, Karlsruhe, GER \\
\hline $\begin{array}{l}\text { ECL (Immobilon TM Western } \\
\text { Chemiluminescent HRP substrate) }\end{array}$ & WBHL50500 & $\begin{array}{l}\text { Merck Millipore, Darmstadt, } \\
\text { GER }\end{array}$ \\
\hline EGTA & 3054.1 & Carl Roth, Karlsruhe, GER \\
\hline Fetal calf serum & $\mathrm{P} 40-47500$ & PAN $^{T M}$ biotech, Passau, GER \\
\hline Glucose & A3594 & Carl Roth, Karlsruhe, GER \\
\hline Glycine & 3908.2 & Carl Roth, Karlsruhe, GER \\
\hline Glycerol & 3783.2 & Carl Roth, Karlsruhe, GER \\
\hline HEPES & P05-01100 & 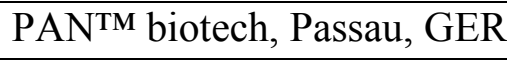 \\
\hline $\mathrm{H}_{2} \mathrm{DCFDA}$ & D399 & $\begin{array}{l}\text { Thermo Scientific, Waltham, } \\
\text { USA }\end{array}$ \\
\hline Horse serum & P30-0702 & PAN $^{T M}$ biotech, Passau, GER \\
\hline Igepal & 18896 & Sigma, St. Louis, USA \\
\hline KCL & 6781.1 & Carl Roth, Karlsruhe, GER \\
\hline $\mathrm{KH}_{2} \mathrm{PO}_{4}$ & 6875.1 & Carl Roth, Karlsruhe, GER \\
\hline $\mathrm{KOH}$ & 6751.3 & Carl Roth, Karlsruhe, GER \\
\hline L-Glutamine & K0283 & Biochrome, Berlin, GER \\
\hline
\end{tabular}




\begin{tabular}{|c|c|c|}
\hline Luminol & 4203.1 & Carl Roth, Karlsruhe, GER \\
\hline Mannitol & 4175.1 & Carl Roth, Karlsruhe, GER \\
\hline Milk Powder & 4021155023078 & Sucofin, Zeven, GER \\
\hline Methanol & 4627.5 & Carl Roth, Karlsruhe, GER \\
\hline $\mathrm{MgCl}_{2}$ & 2189.2 & Carl Roth, Karlsruhe, GER \\
\hline $\mathrm{NaCl}$ & 9265.2 & Carl Roth, Karlsruhe, GER \\
\hline $\mathrm{NaOH}$ & 6771.2 & Carl Roth, Karlsruhe, GER \\
\hline Natriumazide & K305.1 & Carl Roth, Karlsruhe, GER \\
\hline $\begin{array}{l}\text { PageRuler }{ }^{\mathrm{TM}} \text { Prestained Protein } \\
\text { Ladder }\end{array}$ & 221616 & $\begin{array}{l}\text { Thermo Scientific, Waltham, } \\
\text { USA }\end{array}$ \\
\hline Penicillin/streptomycin & P06-0700 & 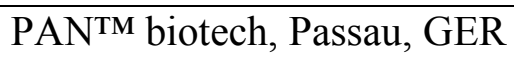 \\
\hline Ponceau-S & P7170 & Carl Roth, Karlsruhe, GER \\
\hline Sodium dodecyl sulfate (SDS) & 4360.2 & Carl Roth, Karlsruhe, GER \\
\hline Sodium Pyruvate & P04-43100 & 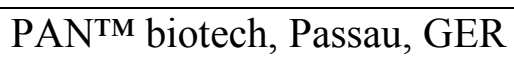 \\
\hline Sucrose & 4661.1 & Carl Roth, Karlsruhe, GER \\
\hline $\begin{array}{l}\text { Tetramethylethylenediamine } \\
\text { (TEMED) }\end{array}$ & 2367.3 & Carl Roth, Karlsruhe, GER \\
\hline $\begin{array}{l}\text { N,N,N }{ }^{\mathrm{I}}, \mathrm{N}^{\mathrm{I}} \text {-Tetramethyl-p- } \\
\text { phenylenediamine (TMPD) }\end{array}$ & T7394 & Sigma, St. Louis, USA \\
\hline Trehalose & 5151.3 & Carl Roth, Karlsruhe, GER \\
\hline Tris & 54292 & Carl Roth, Karlsruhe, GER \\
\hline Triton X-100 & 3051.4 & Carl Roth, Karlsruhe, GER \\
\hline Trizol & 15596026 & Invitrogen, Darmstadt, GER \\
\hline Trypsin/EDTA & P10-023100 & PAN $^{T M}$ biotech, Passau, GER \\
\hline XF-calibration medium & $100840-000$ & $\begin{array}{l}\text { Seahorse Bioscience, North } \\
\text { Billerica, USA }\end{array}$ \\
\hline
\end{tabular}

\subsection{Chemical inhibitors}

The chemical inhibitors used in this project are given in Table 6.

Table 6: Chemical inhibitors

\begin{tabular}{|l|l|l|l|}
\hline Description & $\begin{array}{l}\text { Order } \\
\text { number }\end{array}$ & Manufacturer & $\begin{array}{l}\text { Final } \\
\text { concentration }\end{array}$ \\
\hline DMOG & $\begin{array}{l}\text { BML-E1347- } \\
0050\end{array}$ & $\begin{array}{l}\text { Enzo Biochem, Farmingdale, } \\
\text { USA }\end{array}$ & $1 \mathrm{mM}$ \\
\hline $\begin{array}{l}\text { Dichloroacetate } \\
\text { (DCA) }\end{array}$ & 347795 & Sigma, St. Louis, USA & $5 \mathrm{mM}$ \\
\hline Oligomycin & 495455 & Calbiochem, San Diego, USA & $1.5-3 \mu \mathrm{M}$ \\
\hline FCCP & C2920 & Sigma, St. Louis, USA & $1 \mu \mathrm{M}$ \\
\hline Rotenone & R8875 & Sigma, St. Louis, USA & $2 \mu \mathrm{M}$ \\
\hline
\end{tabular}




\begin{tabular}{|l|l|l|l|}
\hline Antimycin A & A8675 & Sigma, St. Louis, USA & $1 \mu \mathrm{M}$ \\
\hline $\begin{array}{l}\text { 2-deoxyglucose (2- } \\
\text { DG) }\end{array}$ & D6134 & Sigma, St. Louis, USA & $100 \mathrm{mM}$ \\
\hline
\end{tabular}

\subsection{Buffers and solutions}

The buffers and solutions are given in Table 7.

Table 7: Buffers and solutions

\begin{tabular}{|c|c|}
\hline Liquids & Recipe/Manufacturer \\
\hline 2x Laemmli buffer & $\begin{array}{l}100 \mathrm{mM} \text { Tris/HCl, pH } 6.8 \\
4 \% \text { SDS } \\
0.2 \% \text { bromphenol blue } \\
20 \% \text { glycerol } \\
5 \% \beta \text {-mercaptoethanol }\end{array}$ \\
\hline Lysis buffer & $\begin{array}{l}50 \mathrm{mM} \text { Tris } \\
150 \mathrm{mM} \mathrm{NaCl} \\
5 \mathrm{mM} \mathrm{PMSF} \\
100 \mathrm{mM} \mathrm{MgCl}_{2} \\
1 \% \text { Igepal }\end{array}$ \\
\hline ECL & $\begin{array}{l}100 \mathrm{mM} \text { Tris/ } \mathrm{HCl}, \mathrm{pH} 8.5 \\
90 \mathrm{mM} \text { coumaric acid } \\
250 \mathrm{mM} \text { luminol } \\
0.009 \% \mathrm{H}_{2} \mathrm{O}_{2}\end{array}$ \\
\hline PBS (Phosphate Buffered Saline) & $\begin{array}{l}137 \mathrm{mM} \mathrm{NaCl} \\
2.7 \mathrm{mM} \mathrm{KCl} \\
4.3 \mathrm{mM} \mathrm{Na}_{2} \mathrm{HPO}_{4} \cdot 7 \mathrm{H}_{2} \mathrm{O} \\
1.4 \mathrm{mM} \mathrm{KH}_{2} \mathrm{PO}_{4}, \mathrm{pH} 7.4\end{array}$ \\
\hline TBS-T & $\begin{array}{l}20 \mathrm{mM} \text { Tris } \\
150 \mathrm{mM} \mathrm{NaCL} \\
0.1 \%(\mathrm{v} / \mathrm{v}) \text { Tween-20 } \\
\mathrm{pH} 7.6\end{array}$ \\
\hline Running buffer $(1 \mathrm{x})$ & $\begin{array}{l}125 \mathrm{mM} \text { Tris } \\
1.25 \mathrm{M} \text { glycine } \\
0.5 \% \mathrm{SDS}\end{array}$ \\
\hline Transfer buffer $(1 \mathrm{x})$ & $\begin{array}{l}25 \mathrm{mM} \text { Tris } \\
192 \mathrm{mM} \text { glycine } \\
20 \%(\mathrm{v} / \mathrm{v}) \text { methanol }\end{array}$ \\
\hline Developer & $\begin{array}{l}\text { Tetanal (Roentogen) } \\
1: 3.5\end{array}$ \\
\hline Fixer & Tetanal (Roentogen Superfix) \\
\hline
\end{tabular}




\begin{tabular}{|c|c|}
\hline & $1: 4$ \\
\hline THE buffer & $\begin{array}{l}300 \mathrm{mM} \text { trehalose } \\
10 \mathrm{mM} \text { HEPES } \\
10 \mathrm{mM} \text { KCL } \\
1 \mathrm{mM} \text { EDTA } \\
1 \mathrm{mM} \text { EGTA } \\
\text { pH } 7.7\end{array}$ \\
\hline MAS buffer & $\begin{array}{l}70 \mathrm{mM} \text { sucrose } \\
220 \mathrm{mM} \text { mannitol } \\
2 \mathrm{mM} \mathrm{HEPES} \\
10 \mathrm{mM} \mathrm{KH}_{2} \mathrm{PO}_{4} \\
5 \mathrm{mM} \mathrm{MgCl}_{2} \\
1 \mathrm{mM} \mathrm{EGTA} \\
0.2 \% \mathrm{BSA} \\
\text { pH } 7.4 \\
\text { prepare fresh }\end{array}$ \\
\hline Alkaline tail lysis buffer & $\begin{array}{l}25 \mathrm{mM} \mathrm{NaOH} \\
0.2 \mathrm{mM} \text { EDTA }\end{array}$ \\
\hline DCF-assay buffer & $\begin{array}{l}20 \mathrm{mM} \text { Tris } \\
150 \mathrm{mM} \mathrm{NaCl} \\
0.1 \% \text { Triton } \mathrm{pH} 7.4\end{array}$ \\
\hline
\end{tabular}

\subsection{Media}

The media used are given in Table 8 .

Table 8: Media

\begin{tabular}{|l|l|}
\hline Liquids & Recipe/Manufacturer \\
\hline DMEM-Cell Culture Medium & DMEM High Glucose $(4.5 \mathrm{~g} / \mathrm{L})(\mathrm{PAA}$, \\
& Pasching AUS) \\
& $10 \%(\mathrm{v} / \mathrm{v})$ heat-inactivated FCS \\
& $100 \mathrm{U} / \mathrm{mL}$ penicillin $\mathrm{G}$ \\
& $100 \mu \mathrm{g} / \mathrm{mL}$ streptomycin \\
& $200 \mathrm{mM}$ L-glutamine \\
\hline Complete culture medium (BMDM) & DMEM High Glucose $(4.5 \mathrm{~g} / \mathrm{L})(\mathrm{PAA}$, \\
& Pasching AUS) \\
& $10 \%(\mathrm{v} / \mathrm{v})$ heat-inactivated FCS \\
& $200 \mathrm{mM}$ L-glutamine \\
& $1 \mathrm{mM}$ sodium pyruvate, \\
& $1 \mathrm{mM}$ HEPES, \\
& $50 \mathrm{U} / \mathrm{mL}$ penicillin $\mathrm{G}, 50 \mu \mathrm{g} / \mathrm{mL}$ \\
& streptomycin \\
\hline
\end{tabular}




\begin{tabular}{|l|l|}
\hline Pluznik medium & DMEM High Glucose (4.5g/L) (PAA, \\
& Pasching AUS) \\
& $10 \%(\mathrm{v} / \mathrm{v})$ heat-inactivated FCS \\
& $0.2 \mathrm{mM}$ L-glutamine \\
& $0.1 \mathrm{mM}$ sodium pyruvate \\
& $50 \mathrm{U} / \mathrm{mL}$ penicillin $\mathrm{G}, 50 \mathrm{\mu g} / \mathrm{mL}$ \\
& streptomycin \\
& $5 \%(\mathrm{v} / \mathrm{v})$ heat-inactivated horse serum \\
& $0,05 \% 1: 1000$ diluted B-mercaptoethanol \\
& $15 \%(\mathrm{v} / \mathrm{v})$ L929 cell-conditioned medium \\
\hline XF-assay medium & XF assay medium (Seahorse Bioscience, \\
& North Billerica, USA) \\
& $1 \mathrm{mM}$ sodium pyruvate \\
& $4.5 \mathrm{~g} / \mathrm{L}$ glucose \\
& pH 7.35 \\
\hline Glucose-Stresstest-medium (Glycostress- & DMEM D 5030 (Sigma, St. Louis, USA) \\
medium) & $134 \mathrm{mM}$ NaCl \\
& $3 \mathrm{mg}$ phenol red \\
& $2 \mathrm{mM}$ L-glutamine \\
& pH 7.35 \\
\hline
\end{tabular}

\subsection{Antibodies}

The antibodies used in this project are listed in Table 9.

Table 9: Antibodies

\begin{tabular}{|c|c|c|c|c|}
\hline Antibody & Source & Catalog number & Provider & Dilution \\
\hline \multicolumn{5}{|l|}{ Primary Antibodies } \\
\hline anti- $\beta$-actin & mouse & A5441 & $\begin{array}{l}\text { Sigma, St. } \\
\text { Louis, USA }\end{array}$ & $1: 100000$ \\
\hline anti-HIF-1 $\alpha$ & rabbit & NB-100-479 & $\begin{array}{l}\text { Novus, } \\
\text { Colorado, USA }\end{array}$ & $1: 1000$ \\
\hline anti-PHD2 & rabbit & NB 100-2219 & $\begin{array}{l}\text { Novus, } \\
\text { Colorado, USA }\end{array}$ & $1: 500$ \\
\hline anti-PDK1 & rabbit & ADI-KAP-PK112-D & $\begin{array}{l}\text { Stressgene, } \\
\text { British } \\
\text { Columbia, CA }\end{array}$ & $1: 500$ \\
\hline anti-PDH & rabbit & 3205 & $\begin{array}{l}\text { Cell Signaling, } \\
\text { Massachusett, } \\
\text { USA }\end{array}$ & $1: 1000$ \\
\hline anti-p-PDH $\left(\mathrm{pSer}^{293}\right)$ & rabbit & ABS204 & $\begin{array}{l}\text { Merck } \\
\text { Millipore, }\end{array}$ & $1: 10000$ \\
\hline
\end{tabular}




\begin{tabular}{|c|c|c|c|c|}
\hline & & & Darmstadt, GER & \\
\hline \multicolumn{5}{|l|}{ Secondary Antibodies } \\
\hline $\begin{array}{l}\text { anti-rabbit HRP } \\
\text { polyclonal }\end{array}$ & goat & Sc-2004 & $\begin{array}{l}\text { Santa Cruz, } \\
\text { Heidelberg GER }\end{array}$ & $1: 10000$ \\
\hline $\begin{array}{l}\text { anti-mouse HRP } \\
\text { polyclonal }\end{array}$ & goat & Sc-2005 & $\begin{array}{l}\text { Santa Cruz, } \\
\text { Heidelberg GER }\end{array}$ & $1: 1000$ \\
\hline \multicolumn{5}{|l|}{ FACS antibodies } \\
\hline anti-mouse F4/80 PE & rat & 400507 & $\begin{array}{l}\text { Biolegend, San } \\
\text { Diego, USA }\end{array}$ & $1: 500$ \\
\hline $\begin{array}{l}\text { anti-mouse CD11b } \\
\text { APC }\end{array}$ & rat & 101212 & $\begin{array}{l}\text { Biolegend, San } \\
\text { Diego, USA }\end{array}$ & $1: 500$ \\
\hline
\end{tabular}

\subsection{Oligonucleotides}

Oligonucleotides listed in Table 10 were purchased at Biomers, Ulm, GER

Table 10: Oligonucleotides

\begin{tabular}{|c|c|c|}
\hline Gene & \multicolumn{2}{|c|}{ Oligonucleotide } \\
\hline \multicolumn{3}{|l|}{ qPCR } \\
\hline \multirow[t]{2}{*}{$\mathrm{ms} 12$} & forward & 5'-GAAGCTGCCAAGGCCTTAGA -3' \\
\hline & reverse & $3^{\prime}$-AACTGCAACCAACCACCTTC -5' \\
\hline \multirow[t]{2}{*}{ PHD2 } & forward & 5'- TTGCTGACATTGAACCCAAA -3' \\
\hline & reverse & 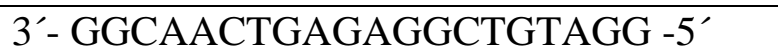 \\
\hline \multirow[t]{2}{*}{ PHD3 } & forward & 5'-GGCCGCTGTATCACCTGTAT -3' \\
\hline & reverse & 3'-TTCTGCCCTTTCTTCAGCAT -5' \\
\hline \multirow[t]{2}{*}{ GLUT1 } & forward & 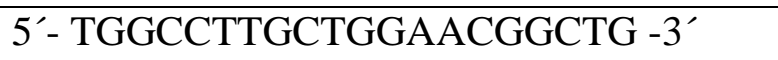 \\
\hline & reverse & $3^{\prime}-$ TCCTTGGGCTGCAGGGAGCA -5' \\
\hline \multirow[t]{2}{*}{ PFK1 } & forward & 5'-ACGAGGCCATCCAGCTCCGT -3' \\
\hline & reverse & 3'- TGGGGCTTGGGCAGTGTCCT -5' \\
\hline \multirow[t]{2}{*}{ PDK1 } & forward & 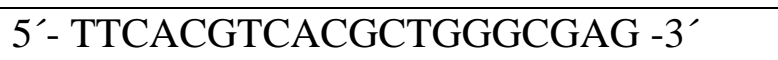 \\
\hline & reverse & 3'-GGCTGGGCACACACCAGTCG -5' \\
\hline \multirow[t]{2}{*}{ Cox4-2 } & forward & 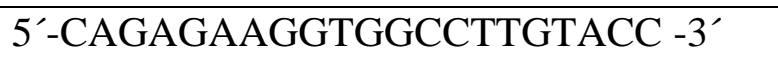 \\
\hline & reverse & 3'-AGAAGAAGACGCAGCCCATC -5' \\
\hline \multirow[t]{2}{*}{ LonP } & forward & 5'-CATCGCCTTGAACCCTCTGT -3' \\
\hline & reverse & 3'-AGCCGCTTAAGGATGTTGGT -5' \\
\hline \multirow[t]{2}{*}{ BNIP3 } & forward & 5'-GTCCAGTGTCGCCTGGCCTC -3' \\
\hline & reverse & 3'-TGGGAGCGAGGTGGGCTGTC -5' \\
\hline \multicolumn{3}{|c|}{ Genotyping } \\
\hline \multirow[t]{2}{*}{ Cre } & forward & 5'- GTTCGCAAGAACCTGATGGACA -3' \\
\hline & reverse & 3'-CTAGAGCCTGTTTTGCACGTTC -5' \\
\hline \multirow{2}{*}{$\begin{array}{l}\text { PHD2 } \\
\text { flox }\end{array}$} & forward & 5’- CTCACTGACCTACGCCGTGT -3’ \\
\hline & reverse & 3'-CGCATCTTCCATCTCCATTT -5' \\
\hline
\end{tabular}




\subsection{Ready-to-use reaction systems}

The ready to use reaction systems listed in Table 11 were utilized in this work.

Table 11: Ready-to-use systems

\begin{tabular}{|c|c|c|}
\hline Description & Order number & Manufacturer \\
\hline L-lactate kit & 10139084035 & R-Biopharm, Darmstadt, GER \\
\hline First Strand cDNA synthesis kit & K1612 & Fermentas St. Leon-Rot, GER \\
\hline $\begin{array}{l}\text { SensiMix }{ }^{\mathrm{TM}} \text { SYBR® Low-ROX } \\
\text { Kit }\end{array}$ & QT625-05 & Bioline, Luckenwalde, GER \\
\hline $\begin{array}{l}\text { CellTiter-Glo® Luminescent } \\
\text { Viability Assay }\end{array}$ & G7570/7571 & Promega, Madison, USA \\
\hline $\begin{array}{l}\text { Pyruvate dehydrogenase- } \\
\text { activity kit }\end{array}$ & MAK183 & Sigma, St. Louis, USA \\
\hline
\end{tabular}

\subsection{Mouse model}

Primary bone marrow derived macrophages were obtained from a myeloid specific PHD2 deficient mouse model (Phd2 floxflox x LysMcre ${ }^{+/}$, PHD2 cKO) (obtained from Ben Wielockx, TU Dresden, described in (Singh et al., 2013)). PHD2 $2^{\mathrm{fl} / \mathrm{fl}}$ mice that contain LoxP sites flanking exon 2 and 3 were crossed with mice that express the Cre recombinase under the control of the endogenous lysozyme M locus (LysMCre) (Jackson Laboratories, Maine, USA, described in (Clausen et al., 1999)). Cre-mediated recombination then resulted in deletion of PHD2 in the myeloid cell lineage, including monocytes, macrophages, granulocytes and in part dendritic cells (Clausen et al., 1999).

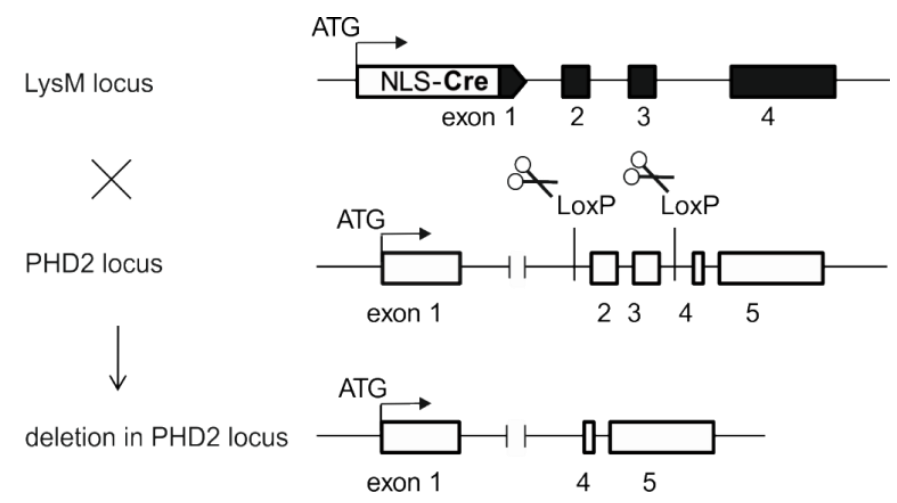

Figure 5: Crossing scheme PHD2 $2^{\mathrm{fl} / \mathrm{fl}}$ mice that contain LoxP sites flanking exon 2 and 3 were crossed with mice that express the Cre recombinase under the control of the endogenous lysozyme M locus (LysMCre). 


\subsection{RAW cell line with constitutively active shRNA targeting PHD2}

The shPHD2 cell line was generated and kindly provided by Dr. B. Wielockx, Institute of Pathology, TU Dresden. RAW 264.7 cells were infected with a lentivirus encoding shRNA targeting mPHD2 (5'-ATTCGAAGAATACCTCCAC-3'). The cells were then used as a pool of sorted EGFP positive cells. Knockdown efficiency was determined by qPCR.

\subsection{Cell biology}

\subsubsection{Cell culturing}

Cells were cultivated in high glucose DMEM medium supplemented with $100 \mathrm{U} / \mathrm{mL}$ penicillin $\mathrm{G}, 100 \mu \mathrm{g} / \mathrm{mL}$ streptomycin and $10 \%$ heat-inactivated fetal calf serum. The cells were kept at $37^{\circ} \mathrm{C}, 5 \% \mathrm{CO}_{2}$ and split every second day in a ratio depending on the observed cell proliferation (mostly in a ratio of 1:10) using a cell scraper. For experiments performed in hypoxia, cells were kept for $24 \mathrm{~h}$ at $37^{\circ} \mathrm{C}$ at $1 \%$ oxygen using the In $\mathrm{Vivo}_{2} 400$ work station. L929 cells and MDA-MB 231 cells were detached using trypsin. The density of cells was determined using a Neubauer counting chamber. The cell number was determined by counting the average cell number of each large square $\mathrm{x} 10^{4} \mathrm{x}$ dilution factor.

To freeze cells, cells were sedimented and resuspended in $1 \mathrm{ml}$ freezing medium (FCS supplemented with $10 \%$ DMSO). Cells were frozen in cryoboxes containing isopropanol to obtain a constant cooling of approximately $1{ }^{\circ} \mathrm{C}$ per minute. To cultivate cells from a frozen aliquot, defrosted cells were washed with $20 \mathrm{ml}$ of the respective medium and sedimented by centrifugation at $450 \mathrm{xg}, 5 \mathrm{~min}$ at RT. The cell pellet was resuspended in $15 \mathrm{ml}$ medium and cells were incubated at $37^{\circ} \mathrm{C}, 5 \% \mathrm{CO}_{2}$ in flasks.

\subsubsection{Preparation of MDA-MB 231 conditioned medium}

5 flasks with each $1 \times 10^{6}$ cells were cultivated in $15 \mathrm{ml}$ complete culture medium for 4 days. The medium was transferred to a reaction tube and centrifuged at $4000 \mathrm{xg}$ for $20 \mathrm{~min}$ at $4{ }^{\circ} \mathrm{C}$. The supernatant was pooled and frozen in $4 \mathrm{~mL}$ aliquots at $-80{ }^{\circ} \mathrm{C}$. 


\subsubsection{Preparation of L929 conditioned medium (Pluznik)}

$4 \times 10^{6}$ L929 cells were seeded in $50 \mathrm{ml}$ complete culture medium in a T75 flask. After 7 days, the supernatant was taken, centrifuged at $4000 \mathrm{xg}$, for $20 \mathrm{~min}$ and filter-sterilized before being stored at $-20^{\circ} \mathrm{C}$.

\subsubsection{Differentiation and cultivation of bone marrow-derived macrophages (BMDM)}

BMDM are primary macrophages isolated from the bone marrow and differentiated in vitro in the presence of macrophage colony-stimulating factor (M-CSF). M-CSF is a growth factor which is important for the proliferation and differentiation of macrophages. In this protocol, the conditioned medium of L929 cell was used as a source of M-CSF (protocol adapted from (Weischenfeldt and Porse, 2008)).

8 to 12 weeks old wild type and PHD2 cKO mice were killed by cervical dislocation and the femur was isolated from both legs. Bone marrow cells were isolated by flushing the femur with a 26 gauge needle and cultured on surface-treated cell culture dishes in complete culture medium (DMEM supplemented with, $0.2 \mathrm{mM}$ L-glutamine, $0.1 \mathrm{mM}$ sodium pyruvate, $1 \mathrm{mM}$ HEPES, $50 \mathrm{U} / \mathrm{mL}$ penicillin $\mathrm{G}, 50 \mu \mathrm{g} / \mathrm{mL}$ streptomycin and $10 \%$ heat-inactivated fetal calf serum) at $37^{\circ} \mathrm{C}$ and $5 \% \mathrm{CO}_{2}$. After $24 \mathrm{~h}$, non-adherent monocytes were harvested and distributed on non-surface-treated cell culture dishes in $10 \mathrm{ml}$ Pluznik's medium (DMEM supplemented with, $0.2 \mathrm{mM}$ L-glutamine, $0.1 \mathrm{mM}$ sodium pyruvate, $50 \mathrm{U} / \mathrm{mL}$ penicillin $\mathrm{G}, 50$ $\mu \mathrm{g} / \mathrm{mL}$ streptomycin $10 \%$ heat-inactivated fetal calf serum, $5 \%$ heat-inactivated horse serum , 0,05\% 1:1000 diluted ß-mercaptoethanol and 15\% L929 cell-conditioned medium) and differentiated for 7 days. On day 3, the Pluznik's medium was refreshed. On day 7 of differentiation, the bone marrow-derived macrophages (BMDM) were detached using $3.5 \mathrm{~mL}$ accutase and seeded for experiments in complete culture medium.

\subsubsection{Efficiency of BMDM differentiation}

BMDM differentiation was checked by analysing F4/80 surface antigen expression using flow cytometry (described in (Weischenfeldt and Porse, 2008)). Cells were detached on day 7 of differentiation using accutase. $0.66 \times 10^{6}$ cells were stained with F4/80-PE labeled antibody for 
20 min in the dark. The cells were washed with PBS and F4/80 expression was analyzed using flow cytometry.

\subsubsection{Isolation and cultivation of murine peritoneal macrophages}

8 to 12 weeks old wild type and PHD2 cKO mice were killed by cervical dislocation. The abdomen was incised without harming the peritoneal cavity. The skin was pulled to the top to show the lining of the peritoneal cavity. The peritoneal cavity was injected with $4 \mathrm{~mL}$ of PBS supplemented with $0.5 \%$ BSA, 2 mM EDTA using a 26 gauge needle. Then, the abdomen was massaged in order to detach cells and with the help of a 25 gauge needle, the cellcontaining PBS was collected. Subsequently, cells were centrifuged at $400 \mathrm{xg}$ for $10 \mathrm{~min}$ at $4^{\circ} \mathrm{C}$, washed once with medium and seeded in 6-well plates. After $2 \mathrm{~h}$, cells were washed twice with medium and cultured in complete culture medium. FACS analysis was performed to estimate the purity of the obtained peritoneal macrophages (see chapter 2.14.5.)

\subsubsection{Mitochondrial preparation}

$5 \times 10^{7}$ cells were washed twice with PBS and harvested in PBS. The cells were sedimented at $1000 \mathrm{xg}$ for $10 \mathrm{~min}$ and the pellet was resuspended in $2 \mathrm{ml}$ THE buffer (300 mM Trehalose, $10 \mathrm{mM}$ HEPES, $10 \mathrm{mM}$ KCL, 1 mM EDTA, 1 mM EGTA, pH 7.7) supplemented with $0.1 \%$ BSA, $1 \mathrm{mM}$ PMSF and 1\% protease inhibitor and homogenized for 25 times in a $2 \mathrm{ml}$ potter, After a centrifugation step of $10 \mathrm{~min}$ at $400 \mathrm{xg}$ at $4^{\circ} \mathrm{C}$, the supernatant was transferred to a new Eppendorf tube and the process was repeated 3 times. Then, the collected supernatant was centrifuged for $7 \mathrm{~min}$ at $800 \mathrm{xg}$ and transferred to a fresh $1.5 \mathrm{ml}$ Eppendorf tube. To pellet the mitochondria, the samples were centrifuged at $11000 \mathrm{xg}$ for $10 \mathrm{~min}$ and the mitochondrial pellet was resuspended in $50 \mu \mathrm{L}$ THE-buffer without BSA. The protein concentration was determined by Bradford assay and diluted to a concentration of $10 \mu \mathrm{g} / \mu \mathrm{L}$. Mitochondria were either used directly or frozen in liquid nitrogen and then stored at $-80{ }^{\circ} \mathrm{C}$ for later use.

\subsubsection{Determination of Lactate levels in BMDM and RAW supernatant}

For the determination of L-lactate levels, a L-lactate kit was used according to the manufacturer's instructions (R-Biopharm). This kit uses the ability of nicotinamide-adenine 
dinucleotide (NAD) to oxidize L-lactate to pyruvate in the presence of L-lactate dehydrogenase (L-LDH) (I).

(I)

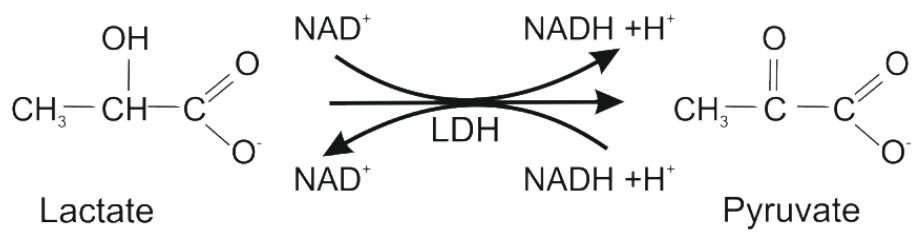

However, the equilibrium of this reaction (I) lies in the favor of L-lactate and NAD ${ }^{+}$. Hence, the pyruvate needs to be trapped. This is achieved using the enzyme glutamate-pyruvate transaminase (GPT). This enzyme converts pyruvate to D-alanine and $\alpha$-ketoglutarate in the presence of L-glutamate (II).

(II)

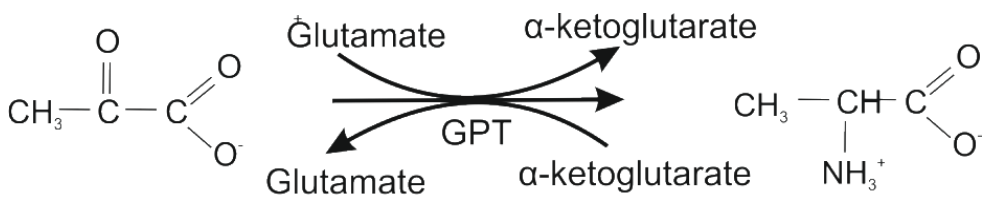

Pyruvate

Alanine

The amount of NADH produced is stoichiometric to the amount of L-lactate in the sample. The increase of NADH was measured spectrophotometrically at $340 \mathrm{~nm}$.

$0.75 \times 10^{5}$ BMDM and RAW cells were cultivated for $24 \mathrm{~h}$ in $24-w e l l$ plates in a volume of $500 \mu \mathrm{L}$ per well. The supernatant was centrifuged at $16000 \mathrm{xg}$ for $30 \mathrm{~min}, 4^{\circ} \mathrm{C}$ to remove cellular debris. 100-150 $\mu \mathrm{L}$ of the supernatant was used for the reaction. An equal amount of medium was used as a blank. The sample was well mixed with the reaction buffer containing $10 \mathrm{ml}$ solution 1 (glycylglycine buffer, pH 10; L-glutaminic acid, ca $440 \mathrm{mg}$ ), $0.2 \mathrm{ml}$ solution 2 (210 mg NAD-lyophilisate), $0.02 \mathrm{ml}$ solution 3 (Glutamate-pyruvate-transaminasesuspension (1100 U)) and $0.9 \mathrm{ml}$ of distilled water. After $5 \mathrm{~min}$, the extinction was measured at $340 \mathrm{~nm}\left(\mathrm{E}_{1}\right)$. Then, $0.02 \mathrm{ml}$ L-lactate-dehydrogenase-solution were added (3800 $\left.\mathrm{U}\right)$ and mixed. After $30 \mathrm{~min}$, the reaction was terminated and the samples were mixed and measured again $\left(E_{2}\right)$. The extinction difference was calculated $\left(E_{2}-E_{1}\right)$. 
The concentration was calculated using the following formula:

$\mathrm{C}=\frac{\mathrm{Vx} M G}{\varepsilon \times d \times v \times 1000} \times \Delta \mathrm{E}(\mathrm{g} / \mathrm{L})$

$\mathrm{V}=$ test volume $(\mathrm{ml})$

$\mathrm{v}=$ sample volume $(\mathrm{ml})$

$\mathrm{MG}=$ molecular weight of substance $(89.1 \mathrm{~g} / \mathrm{mol}$ for L-Lactate $)$

$\mathrm{D}=$ light path $(1 \mathrm{~cm})$

$\mathrm{E}=$ extinction coefficient of NADH at $340 \mathrm{~nm}=6.31 / \mathrm{mmol} \mathrm{x} \mathrm{cm}$

\subsubsection{Measurement of PDH activity}

For the determination of the PDH activity, a kit was used according to the manufacturer's instruction with slight modifications (Sigma-Aldrich). PDH activity was determined in a coupled enzyme reaction measuring the generation of NADH.

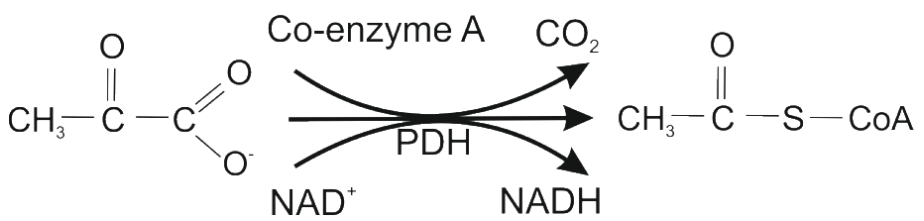

Pyruvate

acetyl-CoA

$5.5 \times 10^{6}$ cells per plate were seeded $24 \mathrm{~h}$ prior to the experiment and treated with the respective inhibitors. The day of the experiment the cells were washed with PBS and harvested in $200 \mu \mathrm{L}$ ice-cold PBS. The cells were then spun down at $10000 \mathrm{xg}$ for $5 \mathrm{~min}$ and washed two more times with PBS before being homogenized in $150 \mu \mathrm{L}$ ice-cold PDH assay lysis buffer. After an incubation time of $10 \mathrm{~min}$, the samples were centrifuged at $10000 \mathrm{xg}$ for 5 min. The supernatant was transferred to a fresh tube. For determination of NADH generation, a NADH standard curve was pipetted in a 96 well plate $(0,2.5,5,7.5,10,12.5$ $\mathrm{nmol} /$ well). $25 \mu \mathrm{L}$ of the samples were used in the assay and each well was filled up to a total volume of $50 \mu \mathrm{L}$ with PDH assay buffer. In parallel, the reaction mix was prepared as described in Table 12.

Table 12: Composition of the reaction mix

\begin{tabular}{|l|l|}
\hline Reagent & Standards and samples \\
\hline PDH assay buffer & $46 \mu \mathrm{L}$ \\
\hline PDH developer & $2 \mu \mathrm{L}$ \\
\hline PDH substrate & $2 \mu \mathrm{L}$ \\
\hline
\end{tabular}


$50 \mu \mathrm{L}$ of the reaction mix was added to each well and mixed well using a horizontal shaker. The plate was incubated at $37^{\circ} \mathrm{C}$ and initially measured after $5 \min \left(\mathrm{T}_{\text {initial }}\right)$ at $450 \mathrm{~nm}$. The plate was continued to be incubated at $37^{\circ} \mathrm{C}$ with measurements every 5 min until the first value of the most active sample exceeded the highest value of the NADH standard curve. At this time, the most active sample is near or higher than the end of the linear range of the standard curve. Therefore, the second to last reading was used for calculating the enzyme activity $\left(\mathrm{T}_{\text {final }}\right)$. First, the initial measurements $\left(\mathrm{T}_{\text {initial }}\right)$ were subtracted from the final readings $\left(\mathrm{T}_{\text {final }}\right)$.

$\mathrm{S}_{\mathrm{a}}(\Delta \mathrm{A} 450)=(\mathrm{A} 450)_{\text {final }}-(\mathrm{A} 450)_{\text {initial }}$.

With the corrected measurements, the amount of NADH (nmol/well) produced by the PDH assay was calculated between $\mathrm{T}_{\text {initial }}$ and $\mathrm{T}_{\text {final }}(\mathrm{Sa})$.

$$
\text { PDH activity }=\frac{\mathrm{Sa}}{\text { Reaction time } \mathrm{x} \mathrm{Sv}}
$$

With:

$\mathrm{S}_{\mathrm{a}}=$ amount of NADH (nmol) generated in unknown sample well between $\mathrm{T}_{\text {initial }}$ and $\mathrm{T}_{\text {final }}$ from standard curve

Reaction Time $=\mathrm{T}_{\text {final }}-\mathrm{T}_{\text {initial }}(\mathrm{min})$

$\mathrm{S}_{\mathrm{v}}=$ sample volume $(\mathrm{mL})$ added to well

The activity of PDH was reported as $\mathrm{nmol} / \mathrm{min} / \mathrm{mL}=\mathrm{milliU} / \mathrm{mL}$. One unit is defined as the amount of enzyme needed to generate $1 \mu \mathrm{mol}$ of NADH per min at $37^{\circ} \mathrm{C}$.

Additionally, the protein concentration of the samples was determined by Bradford assay and used for normalization.

\subsubsection{ATP levels}

For measurements of cellular ATP levels, $0.25 \times 10^{5}$ BMDM or RAW cells were seeded in $100 \mu \mathrm{L}$ culture medium in 96-well luminometer plates for cell culture and measured $24 \mathrm{~h}$ later using the ATP kit according to the manufacturer's instructions with slight adaptations (Promega). The Cell titer-Glo® Assay relies on the properties of thermostable luciferase which converts beetle luciferin to oxyluciferin in the presence of ATP. After pipetting an ATP standard curve to each plate and after adding the lysis buffer, cells were kept at $37{ }^{\circ} \mathrm{C}$ for 5 min to allow cells to lyse before taking them out of the hypoxia chamber or the incubator. The plates were incubated for $30 \mathrm{~min}$ on a shaker, then assayed in a luminometer plate reader and normalized to protein content as determined by Bradford assay. 


\subsubsection{Seahorse measurements}

\subsubsection{Measurement of oxygen consumption rate of total cells using the Seahorse} XF96 analyzer

The Seahorse XF96 analyzer (SeahorseBioscience) was used to determine the oxygen consumption rate (OCR). This is done by measuring the quenching of an oxygen-sensitive fluorophore. The Seahorse XF96 analyzer can be used to assess cellular functions such as oxidative phosphorylation by metabolically disturbing the cells. The first addition is oligomycin. Oligomycin blocks the proton channel of the ATP synthase thereby inhibiting ATP synthesis. It is used to distinguish between the OCR used for ATP synthesis and OCR needed to overcome the natural proton leak across the inner mitochondrial membrane.

The second addition is FCCP. FCCP is used as an uncoupling agent. It transports protons across the mitochondrial membrane. This disrupts the mitochondrial membrane potential leading to a fast increase in OCR without producing ATP. Thus, FCCP is used to determine the maximal respiratory capacity of the cells.

The third addition is the combination of the complex I inhibitor rotenone and the complex III inhibitor antimycin A. Rotenone inhibits the transfer of electrons from complex I to ubiquinone. Antimycin A binds to the cytochrome c reductase. This way, the oxidation of ubiquinol is prevented. As a result, mitochondrial respiration is arrested. The residual respiration is of non-mitochondrial origin and needs to be deducted from the rest of the measured rates.

$2.5 \times 10^{4}$ RAW or $4.0 \times 10^{4}$ BMDM cells per well were seeded the day prior to the experiment in a XF96 cell culture microplate in the respective cell culture medium. The XF sensor is hydrated with XF Buffer at $37^{\circ} \mathrm{C}$ without $\mathrm{CO}_{2}$ overnight. At the day of the experiment, the injection ports of the XF-sensor cartridge were loaded with the compounds noted in Table 13 and the sensor cartridge was placed in the XF96 analyzer for calibration. 
Table 13: Concentration of compounds

\begin{tabular}{|l|l|l|}
\hline Port & Reagent & Concentration (final) \\
\hline A & XF-assay medium & n/a \\
\hline B & oligomycin & $1.5 \mu \mathrm{M}$ \\
\hline C & FCCP & $1 \mu \mathrm{M}$ \\
\hline D & rotenone & $2 \mu \mathrm{M}$ \\
\cline { 2 - 3 } & antimycin A & $1 \mu \mathrm{M}$ \\
\hline
\end{tabular}

In parallel, the medium of the XF cell culture microplate was replaced with XF assay medium supplemented with $4.5 \mathrm{~g} / \mathrm{L}$ Glucose and $1 \mathrm{mM}$ sodium pyruvate and incubated at $37{ }^{\circ} \mathrm{C}$ without $\mathrm{CO}_{2}$ for 30 min. Oxygen consumption was measured using the protocol shown in the Table 14.

Table 14: Measurement protocol for OCR

\begin{tabular}{|l|l|}
\hline Command & Time (min) \\
\hline calibrate & -- \\
\hline equilibrate & -- \\
\hline measure & 4 \\
\hline mix & 2 \\
\hline measure & 4 \\
\hline Inject Port A & \multicolumn{2}{|l|}{} \\
\hline mix & 2 \\
\hline measure & 4 \\
\hline mix & 2 \\
\hline measure & 4 \\
\hline Inject Port B & \multicolumn{2}{|l|}{} \\
\hline mix & 3 \\
\hline measure & 4 \\
\hline mix & 2 \\
\hline measure & 4 \\
\hline Inject Port C & \multicolumn{1}{|l|}{} \\
\hline mix & 1 \\
\hline measure & 4 \\
\hline mix & 2 \\
\hline measure & 4 \\
\hline Inject Port D & 3 \\
\hline mix & 4 \\
\hline measure & 2 \\
\hline mix & 4 \\
\hline measure & \\
\hline &
\end{tabular}




\subsubsection{Measurement of the extracellular acidification rate using Seahorse XF96 analyzer}

The conversion of glucose to pyruvate and subsequently to lactate results in the production and extrusion of protons. This leads to the acidification of the medium. This acidification can be measured by the XF96 analyzer and is reported as extracellular acidification rate (ECAR) (SeahorseBioscience). The cells were seeded at a density from $2.5 \times 10^{4}$ (RAW) $-4 \times 10^{4}$ (BMDM) cells per well. The next day, the cells were washed 10 times with XF Glycostressmedium (DMEM D 5030, 134 mM NaCl, 3 mg Phenol Red, 2 mM L-glutamine, pH 7.35). XF-Glycostress-medium does not contain either glucose or pyruvate, but only $1 \mathrm{mM} \mathrm{L-}$ Glutamine. The cells were incubated in $180 \mu \mathrm{L}$ of XF Glycostress-medium for $15 \mathrm{~min}$ at $37^{\circ} \mathrm{C}$ without $\mathrm{CO}_{2}$. In parallel, the XF sensor cartridge was prepared. The protocol used for the XF measurement is shown in Table 15.

The first addition is a saturating concentration of $10 \mathrm{mM}$ freshly prepared glucose. The cells use the injected glucose and catabolize it using the glycolytic pathway leading to an increase in proton production. This causes an increase in the ECAR. This glucose-induced response is referred to as the glycolytic rate under basal conditions. The second addition is $1.5 \mu \mathrm{M}$ (for RAW cells) or $3 \mu \mathrm{M}$ (for BMDM) oligomycin. Oligomycin blocks the mitochondrial ATP production which causes a shift of the energy production in favor of glycolysis. This rapidly increases the ECAR and reveals the maximal glycolytic capacity of the cell. The last addition is $100 \mathrm{mM}$ of the glucose analogue 2-deoxy-glucose (2-DG). 2-DG inhibits glycolysis because of its competitive binding to glucose hexokinase. This results in a decrease in ECAR and confirms that the ECAR measured in the experiment is in fact due to glycolysis.

Table 15: Measurement protocol for ECAR

\begin{tabular}{|l|l|l|}
\hline Command & & Time (min) \\
\hline calibrate & & -- \\
\hline equilibrate & & -- \\
\hline loop 3 times & mix & 2 min \\
\hline & measure & 4 min \\
\hline loop end & \multicolumn{2}{l|}{} \\
\hline Inject port A & mix & 2 min \\
\hline loop 3 times & measure & 4 min \\
\hline \multicolumn{2}{|l|}{} \\
\hline loop end & & \\
\hline
\end{tabular}




\begin{tabular}{|c|c|c|}
\hline \multicolumn{3}{|l|}{ Inject port $B$} \\
\hline loop 3 times & $\operatorname{mix}$ & $2 \mathrm{~min}$ \\
\hline & measure & $4 \mathrm{~min}$ \\
\hline loop end & $\operatorname{mix}$ & $2 \mathrm{~min}$ \\
\hline \multicolumn{3}{|l|}{ Inject port $\mathbf{C}$} \\
\hline \multicolumn{3}{|l|}{ loop 3 times } \\
\hline & measure & $4 \mathrm{~min}$ \\
\hline \multicolumn{3}{|l|}{ loop end } \\
\hline Program end & & \\
\hline
\end{tabular}

\subsubsection{Measurement of phagocytosis by flow cytometry}

Phagocytic capacity was determined by investigating the uptake of fluorescent beads by flow cytometry. Flow cytometry is based on the detection of fluorescence emitted by a single cell flowing through a liquid stream (described in (Jaroszeski and Radcliff, 1999). A laser beam of a specific wavelength is pointed to the liquid stream. Several detectors are placed at the point where the stream passes the laser beam. One detector is in line with the laser beam and provides information about the Forward Scatter (FSC) of the cell and several others are placed vertically to it measuring the Side Scatter (SSC). Every cell that passes the laser beam scatters the light. The FSC depends on the cell volume and the SSC correlates with the inner complexity (or granularity) of the cell. Furthermore, there are also fluorescent detectors which detect fluorescence emitted by the cell. For the detection of phagocytosis, phycoerythrin (PE)-labeled beads (absorption maximum at $494 \mathrm{~nm}$, emission maximum of $575 \mathrm{~nm}$, Biomol) were used.

$0.33 \times 10^{6}$ RAW cells or $0.66 \times 10^{6}$ BMDMs were seeded in $3.5 \mathrm{~cm}$ dishes and incubated at normoxic and hypoxic conditions overnight. RAW cells were seeded on cell culture-treated plates and BMDM on non-surface-treated plates. Fluorescent beads were added to the cells and incubated for $4 \mathrm{~h}$. RAW cells were detached using dispase and BMDMs using accutase. Cell were washed twice with PBS and then analyzed by flow cytometry.

\subsubsection{Measurement of reactive oxygen species (ROS) by $\mathrm{H}_{2}-\mathrm{DCF}$}

For the determination of mitochondrial reactive oxygen species (ROS), $150 \mu \mathrm{g}$ isolated mitochondria were measured in $500 \mu \mathrm{L}$ assay buffer $(20 \mathrm{mM}$ Tris, $150 \mathrm{mM} \mathrm{NaCl}, 0.1 \%$ Triton, $\mathrm{pH} 7.4)$ with $200 \mu \mathrm{M} 2^{\prime}, 7^{\prime}$-dichlorodihydrofluorescein diactetate $\left(\mathrm{H}_{2} \mathrm{DCFDA}\right)$ at $37^{\circ} \mathrm{C}$ 
in a Hitachi F-7000 fluorimeter with an excitation wavelength of $495 \mathrm{~nm}$ and an emission wavelength of $525 \mathrm{~nm}$ for $1000 \mathrm{sec} . \mathrm{H}_{2}$ DCFDA is non fluorescent, but when oxidized it is converted to the fluorescent 2',7-dichlorofluorescin (DCF). Basal reading (buffer only) was subtracted from the other measurements and fluorescence was depicted as arbitrary units (a.u.).

\subsubsection{Measurement of reactive oxygen species by DHE-HPLC}

Dihydroethidium (5-ethyl-5,6-dihydro-6-phenyl-3,8-diaminophenanthridine, DHE) is an hydrophobic uncharged compound which becomes positively charged upon oxidation. It intercalates with the DNA of the cells. Determination of DHE fluorescence can be used as a measure for reactive oxygen species (ROS) in cells. DHE can be oxidized to two different products (2-hydroxyethidium (EOH) and ethidium). These products can be separated by DHE-HPLC (protocol adapted from (Laurindo et al., 2008). Cells were washed two times with PBS and incubated with $100 \mu \mathrm{M}$ DHE for $30 \mathrm{~min}$ in $500 \mu \mathrm{L}$ PBS + $0.1 \mathrm{mM}$ DPTA. The cells were then harvested in $500 \mu \mathrm{L}$ cold acetonitrile and sonicated for $10 \mathrm{~s}, 1$ cycle at 6-8 W. Acetonitrile is an organic solvent which efficiently breaks the interaction of DHE products and DNA. Then, the cell lysates were spun at $12000 \mathrm{xg}$ for $10 \mathrm{~min}$ at $4^{\circ} \mathrm{C}$. After that, supernatants were dried under vacuum. Pellets were either stored at $-20{ }^{\circ} \mathrm{C}$ in the dark or directly re-suspended for measurement in $80 \mu \mathrm{L}$ PBS $+0.1 \mathrm{mM}$ DTPA. Samples were analyzed by high performance liquid chromatography (HPLC). 2-hydroxyethidine (EOH) and ethidium were separated using a Kromasil $\mathrm{C}_{18}$ column (particle size: $5 \mu \mathrm{m}$, dimensions: $4.6 \times 250 \mathrm{~mm}$ ) in a HPLC system containing a photodiode array and fluorescence detectors. $30 \mu \mathrm{L}$ of each sample was injected. EOH and ethidium was identified detecting fluorescence at an excitation wavelength of $510 \mathrm{~nm}$ and an emission wavelength of $595 \mathrm{~nm}$. A gradient of solution A (pure acetonitrile) to solution B (water/ $10 \%$ acetonitrile/ $0.1 \%$ trifluoroacetic acid, v/v/v) were used at a flow rate of $0.4 \mathrm{ml} / \mathrm{min}$. Runs started with $0 \%$ solution $\mathrm{A}$, then this is increased up to $40 \%$ solution A during the first $10 \mathrm{~min}$. This condition was maintained for 10 more min and then changed to $100 \%$ solution A for additional 5 min and $0 \%$ solution A for $10 \mathrm{~min}$. The unoxidized DHE was measured by UV absorption at $245 \mathrm{~nm}$. Therefore, the remaining DHE was subtracted from the initial DHE concentrations. DHE-products were expressed as ratios of EOH and ethidium generated per consumed DHE (EOH/DHE and ethidium/DHE). 


\subsubsection{Cell proliferation assay}

$2 \times 10^{4}$ cells per cell line were seeded in $2 \mathrm{ml}$ in $3.5 \mathrm{~cm}$ dishes. Cells were detached by thorough scraping at day 1, 3, 5 and 7 after being seeded. Viable cells were stained using trypan blue and counted using a Neubauer chamber. When reaching a seeding density of ca $70 \%$, cells were transferred to a $6 \mathrm{~cm}$ dish and subsequently a $10 \mathrm{~cm}$ dish. The cell doubling time (DT) was calculated using the formula:

$\mathrm{DT}=\frac{\text { duration }(d) * \log (2)}{\log (\text { Nfin })-\log (\text { Ninit })}$ $\mathrm{d}=$ days

$\mathrm{N}_{\text {fin }}=$ cell number at the end of the experiment

$\mathrm{N}_{\text {init }}=$ cell number at the beginning of the experiment

\subsubsection{Migration assays}

\subsubsection{Boyden chamber}

The Boyden chamber assay is based on a chamber of two compartments that are separated by a microporous membrane (described in (Chen, 2005)). The cells are placed in the upper compartment. In the lower compartment a chemotactic stimulus is present. The cells can migrate from the upper compartment to the lower compartment toward the chemotactic stimulus. In this study, the supernatant of MDA-MB 231 cells, a breast cancer cell line, was used as a migration stimulus.

$0.7 \times 10^{5}$ BMDM and RAW cells were placed in a cylindrical cell culture insert with a pore size of $3 \mu \mathrm{m}$ (BD Biosciences) in a volume of $500 \mu \mathrm{L}$ in a 24 well plate. In the well below, the supernatant of MDA-MB 231 cells was placed. After $18 \mathrm{~h}$, the cells which migrated to the lower chamber were stained with $5 \mu \mathrm{M}$ calcein (Sigma, St. Louis, USA) and the cells from the upper chamber were gently removed using a cotton swab. Transwell-migration was analyzed by microscopy at 10x magnification. 7 images from each upper edge to the lower edge of the wells were taken. For analysis, the images at the edges were excluded and only the 5 middle images were used. The amount of migrated cells was analyzed using the "find maxima"-function of ImageJ. 


\subsubsection{Single cell migration}

$2.5 \times 10^{4}$ RAW cells and $5 \times 10^{4}$ BMDMs were seeded in a 6 well plate in $2 \mathrm{ml}$. The next day, the medium was replaced with either normal cell culture medium or MDA-MB 231 conditioned medium. The migration of the cells was investigated using the T1-5M Nikon microscope inside the Sci-tive box at normoxic or hypoxic conditions. An image was taken every $10 \mathrm{~min}$ for $6 \mathrm{~h}$. The migration was analyzed using the manual tracking plugin (http://rsb.info.nih.gov/ij/plugins/manual-tracking.html) and chemotaxis plugin (http://ibidi.com/software/chemotaxis_and_migration_tool/) installed in ImageJ.

\subsection{Molecular biology}

\subsubsection{Genotyping}

Small pieces of mouse ear or tail were placed into $75 \mu \mathrm{L}$ of alkaline lysis buffer $(25 \mathrm{mM}$ $\mathrm{NaOH}, 0.2 \mathrm{mM}$ EDTA). For the genomic DNA (gDNA) of RAW cells and BMDMs, $0.75 \times 10^{6}$ cells were lysed in $75 \mu \mathrm{L}$ alkaline lysis buffer. Then the samples were incubated for 1 hour at $95^{\circ} \mathrm{C}$ to lyse the tissue. The samples were transferred to $4^{\circ} \mathrm{C}$ for $10 \mathrm{~min}$. Subsequently, $75 \mu \mathrm{l}$ of $40 \mathrm{mM}$ Tris/ $\mathrm{HCl}$ buffer was added. $1 \mu \mathrm{l}$ of each sample was used for the PCR reaction. gDNA isolated from RAW cells and BMDMs was used for qPCR (see chapter 2.15.4). The protocol for genotyping is found in Table 16. The primers used for genotyping can be found in Table 16 .

Table 16: Genotyping PCR

\begin{tabular}{|c|c|c|c|c|c|}
\hline & \multicolumn{2}{|l|}{ Cre } & \multicolumn{2}{|l|}{ PHD2 flox } & \multirow[b]{2}{*}{ Repeats } \\
\hline Step & Temperature & Time & Temperature & Time & \\
\hline $\begin{array}{l}\text { Initial } \\
\text { denaturation }\end{array}$ & $94^{\circ} \mathrm{C}$ & $240 \mathrm{sec}$ & $94{ }^{\circ} \mathrm{C}$ & $120 \mathrm{sec}$ & 1 cycle \\
\hline Denaturation & $94^{\circ} \mathrm{C}$ & $30 \mathrm{sec}$ & $94^{\circ} \mathrm{C}$ & $15 \mathrm{sec}$ & \multirow[t]{3}{*}{30 cycles } \\
\hline Annealing & $60^{\circ} \mathrm{C}$ & $30 \mathrm{sec}$ & $53^{\circ} \mathrm{C}$ & $25 \mathrm{sec}$ & \\
\hline Elongation & $72^{\circ} \mathrm{C}$ & $75 \mathrm{sec}$ & $72^{\circ} \mathrm{C}$ & $30 \mathrm{sec}$ & \\
\hline
\end{tabular}




\subsubsection{RNA extraction}

For RNA isolation $0.75 \times 10^{6} \mathrm{BMDM}$ or $0.66 \times 10^{6} \mathrm{RAW}$ cells were washed twice with PBS and harvested in $500 \mu \mathrm{L}$ Trizol (Invitrogen). Then, $100 \mu \mathrm{L}$ chloroform was added, vortexed and kept at RT for $5 \mathrm{~min}$. After a centrifugation step of $12000 \mathrm{xg}$ for $10 \mathrm{~min}$ at $4^{\circ} \mathrm{C}$, the upper layer (aqueous phase) was transferred to a fresh Eppendorf tube with $250 \mu \mathrm{L}$ of isopropanol. After $10 \mathrm{~min}$ of incubation at RT, the eppendorf tubes were centrifuged at $12000 \mathrm{xg}$ for 10 $\min , 4^{\circ} \mathrm{C}$. This was followed by two washing steps with $500 \mu \mathrm{L}$ and $750 \mu \mathrm{L} 75 \%$ EtOH. After centrifugation for $10 \mathrm{~min}$ at $12000 \mathrm{xg}, 4^{\circ} \mathrm{C}$, the supernatant was removed and the pellet left to dry for 10 min with the lid of the Eppendorf cup opened. Then $15 \mu \mathrm{L}$ (for RNA from BMDM) or $40 \mu \mathrm{L}$ (for RNA from RAW cells) of nuclease-free water was added and the pellet was resolved for $10 \mathrm{~min}$ at $56^{\circ} \mathrm{C}$. Afterwards, the samples were either frozen at $-80^{\circ} \mathrm{C}$ or used directly for cDNA synthesis.

\subsection{3. cDNA-Synthesis}

cDNA is a DNA copy that is synthesized from messenger RNA (mRNA) (reviewed in (Harbers, 2008). This reaction is mediated by a reverse transcriptase, which is a retroviral RNA-dependent DNA polymerase. As a starting point for synthesis, the poly (A) tail found at the $3^{\prime}$-end of most eukaryotic mRNA is used. To generate a starting point, a short complementary synthetic oligonucleotide (Oligo-dT primer) is hybridized. Using the four deoxynucleotide triphosphates and in the presence of magnesium ions and at a neutral $\mathrm{pH}$, the reverse transcriptase synthesises complementary DNA from the original mRNA.

$2 \mu \mathrm{g}$ RNA was transcribed with the First Strand cDNA Synthesis Kit (Fermentas). For cDNA synthesis, the RNA was prepared in a volume of $11 \mu \mathrm{L}$ together with $1 \mu \mathrm{L}$ Oligo-dT-Primer and incubated at $65^{\circ} \mathrm{C}$ for $10 \mathrm{~min}$. The samples were chilled on ice for $5 \mathrm{~min}$, shortly spun down and placed back on ice. Then $9 \mu \mathrm{L}$ reaction buffer was added (containing $4 \mu \mathrm{L} 5 \mathrm{x}$ Reaction buffer, $1 \mu \mathrm{L}$ RiboLock RNase Inhibitor (20 U/ $\mu \mathrm{L}), 2 \mu \mathrm{L} 10 \mathrm{mM}$ dNTP-Mix and $2 \mu \mathrm{L}$ M-MuLV Reverse Transcriptase $(20 \mathrm{U} / \mu \mathrm{L}))$. After gentle mixing and centrifugation, the samples were incubated for $60 \mathrm{~min}$ at $37^{\circ} \mathrm{C}$. The reaction was terminated by heating at $70{ }^{\circ} \mathrm{C}$ for $5 \mathrm{~min}$. The synthesized cDNA was either used directly or stored at $-20^{\circ} \mathrm{C}$. 


\subsubsection{Transcript quantification by qRT-PCR (quantitative Real-Time-Polymerase Chain Reaction)}

Quantitative Real-Time-PCR can be used to amplify and quantify simultaneously a targeted DNA sequence in a double stranded DNA molecule (reviewed (Wilhelm and Pingoud, 2003). It uses primer pairs of each about 20 nucleotides in length that are complementary to the targeted sequence on each of the two strands of the DNA. A DNA polymerase binds to the annealed primers copying the targeted sequence. After copying, the primers bind to the originally DNA molecule as well as to the newly synthesized copy. This leads to an exponential amplification. SYBR® Green is an asymmetrical cyanine dye which is used as a nucleic acid stain because of its binding affinity to double stranded DNA (dsDNA). The SYBR ${ }^{\circledR}$ Green-dsDNA complex absorbs blue light at a wavelength of $\lambda_{\max }=488 \mathrm{~nm}$ and emits green light at a wavelength of $\lambda_{\max }=522 \mathrm{~nm}$. This property can be used to detect doublestranded PCR products during the PCR reaction. As a reference dye, ROX ${ }^{\mathrm{TM}}$ was used to normalize the fluorescence between the reactions. The number of PCR cycles that show a significant exponential increase in fluorescence correlates with the amount of copied DNA templates. This is referred to as Cycle Threshold (CT).

Transcript quantification was carried out with SensiMix ${ }^{\text {TM }}$ SYBR ${ }^{\circledR}$ Low-ROX Kit (Bioline). The PCR reaction was performed using MX3005Pro light cycler sequence detection system. For each qRT PCR-reaction, $12.5 \mu \mathrm{L}$ SYBR® GreenMix was used and an amount of cDNA corresponding to $10 \mathrm{ng}$ RNA $(1 \mu \mathrm{L})$ and $400 \mathrm{nM}$ forward and reverse primer in total reaction volume of $25 \mu \mathrm{L}$. PCR reactions were performed either in a 96 well plate sealed with an optical adhesive foil or in PCR reaction tubes. The reaction was performed using the protocol in Table 17. The $\mathrm{T}_{\mathrm{m}}$ value differs because it depends on the melting temperature of the PCR product. The $\mathrm{T}_{\mathrm{m}}$ is defined as the temperature where $50 \%$ of the helices are dissociated because the DNA helix melts in a temperature range rather than at one specific temperature. 
Table 17: qPCR protocol

\begin{tabular}{|l|l|l|l|}
\hline Step & Temperature & Time & Repeats \\
\hline Initial denaturation & $95^{\circ} \mathrm{C}$ & $10 \mathrm{~min}$ & 1 cycle \\
\hline Denaturation & $95^{\circ} \mathrm{C}$ & $30 \mathrm{sec}$ & \multirow{2}{*}{40 cycles } \\
\hline Annealing & $\mathrm{T}_{\mathrm{m}}^{\circ} \mathrm{C}$ & $20 \mathrm{sec}$ & \\
\hline Extension & $72^{\circ} \mathrm{C}$ & $30 \mathrm{sec}$ & \multirow{2}{*}{1 cycle } \\
\cline { 1 - 3 } Dissociation stage & $95^{\circ} \mathrm{C}$ & $60 \mathrm{sec}$ & \\
\cline { 2 - 3 } & $\mathrm{T}_{\mathrm{m}}{ }^{\circ} \mathrm{C}$ & $30 \mathrm{sec}$ & \\
\cline { 2 - 3 } & $95^{\circ} \mathrm{C}$ & $30 \mathrm{sec}$ & \\
\hline
\end{tabular}

The primer sequences used are listed in Table 10.

The relative expression value of each gene was estimated by normalization of the probe CT values to CT values of the housekeeper gene mouse ribosomal protein S12 (ms12).

$\Delta \mathrm{CT}=\mathrm{CT}_{\text {gene of interest }}{ }^{-\mathrm{CT}}$ housekeeping gene

The control and modified samples can be compared by calculating $\Delta \Delta \mathrm{CT}$ values. $\Delta \Delta \mathrm{CT}$ indicates the difference of cycles between the modified and the control condition. The $\Delta \Delta \mathrm{CT}$ is used to calculate the normalized gene expression level $\left(2^{-\Delta \mathrm{CT}}\right)$. This can be used to calculate the fold change of the modified sample compared to the mean of the control condition.

For the determination of the mitochondrial content, a standard curve for the nuclear genomic DNA marker $18 \mathrm{~S}\left(1 \times 10^{2}-1 \times 10^{8}\right.$ copies $)$ and the mitochondrial DNA marker mtCO2 $\left(1 \times 10^{5}\right.$ $1 \times 10^{10}$ copies) was included. 


\subsection{Protein biochemistry}

\subsubsection{Protein extraction}

Cells were washed twice with ice-cold PBS and then lysed in protein lysis buffer $(50 \mathrm{mM}$ Tris, $150 \mathrm{mM} \mathrm{NaCl}, 5 \mathrm{mM}$ PMSF, $100 \mathrm{mM} \mathrm{MgCl}_{2}, 1 \%$ Igepal) containing protease inhibitor cocktail cOmplete Mini. For the detection of phosphorylated protein, the phosphatase inhibitor cocktail PhosStop was used. The cells were detached from the plates using a cell scraper and incubated on ice for $10 \mathrm{~min}$. The extract was centrifuged for $20 \mathrm{~min}$ at $4^{\circ} \mathrm{C}$ at $16000 \mathrm{xg}$. The supernatant was transferred to a fresh tube and the protein concentration was determined by using the Bradford protein assay reagent.

\subsubsection{Bradford assay}

The Bradford protein assay is a colorimetric protein assay. At a low $\mathrm{pH}$, the used dye Coomassie Blue $\mathrm{G}$ is present in a protonated form and has a red color. When protein binds, the dye donates the protons and the color shifts to blue with an absorbance maximum of $595 \mathrm{~nm}$. The increase of absorbance at $595 \mathrm{~nm}$ is then proportional to the amount of protein present in the sample. A standard curve was created with bovine serum albumin (BSA) concentrations ranging from $0.5 \mu \mathrm{g}$ to $6 \mu \mathrm{g}$ to calculate the protein concentration of the samples. Then, $200 \mu \mathrm{l}$ Bradford protein assay reagent was added. The protein concentrations were determined by measuring the absorption at $595 \mathrm{~nm}$ using a microplate reader (Model 680, Bio-Rad) and calculated via the Microplate Manager® program.

\subsubsection{SDS Page}

SDS-Polyacrylamide-electrophoreses enables to separate proteins in an electric field based on their size. A discontinuous system consisting of separation and stacking gel was used. The running buffer contains SDS (sodium dodecyl sulphate) breaks up the non-covalent bonds and thereby leads to denaturation of the protein. The anionic properties of SDS lead to a negative charge of the SDS-protein-complex. This way, the speed of migration is only dependent on the size of the protein. Additionally, reducing reagents like $\beta$-mercaptoethanol are used to break down disulfide bonds and thus disrupt the protein structure. In the stacking gel ( $\mathrm{pH} 6.8$ ), the proteins are brought to a consistent front. They migrate between the fast migrating 
chloride-ions and the slower migrating glycine-ions. In the separation gel $(\mathrm{pH}=8.8)$, glycin ionizes and passes the protein samples. The proteins can therefore move depending on size. SDS-PAGE gels were used in concentrations of $10 \%$ and $12 \%$ for resolving gels and $5 \%$ for the stacking gel (according to Table 18). The resolving gel was prepared first and covered with isopropanol. After polymerization, the stacking gel poured on top of the resolving gel after having removed the isopropanol.

Table 18: Composition of SDS PAGE gels

\begin{tabular}{|l|l|l|l|}
\hline & stacking gel $(\mathbf{m L})$ & \multicolumn{2}{l|}{ resolving gel $(\mathbf{m L})$} \\
\cline { 2 - 4 } & $\mathbf{5 \%}$ & $\mathbf{1 0 \%}$ & $\mathbf{1 2 \%}$ \\
\hline $\mathrm{H}_{2} \mathrm{O}$ & 6.8 & 9.9 & 8.2 \\
\hline $30 \%$ acrylamide mix & 1.7 & 8.3 & 10 \\
\hline $1.5 \mathrm{M}$ Tris pH 8.8 & - & 6.3 & 6.3 \\
\hline 1 M Tris pH 6.8 & 1.25 & - & - \\
\hline $10 \%$ SDS & 0.1 & 0.25 & 0.25 \\
\hline $10 \%$ ammonium persulfate & 0.1 & 0.25 & 0.25 \\
\hline TEMED & 0.01 & 0.01 & 0.01 \\
\hline
\end{tabular}

The protein sample (containing $80-100 \mu \mathrm{g}$ protein) was supplemented with Laemmli loading buffer (100 mM Tris pH 6.8, 4\% SDS, 0.2\% bromophenol blue, 20\% glycerol, 5\% $\beta$ mercaptoethanol), denaturized $\left(5 \mathrm{~min}, 95^{\circ} \mathrm{C}\right)$ and loaded onto the SDS Page gel. The gels were run at a current of $40 \mathrm{~mA}$. For protein size determination, the PageRuler ${ }^{\mathrm{TM}}$ marker was used. 


\subsubsection{Immunoblotting}

For detection of proteins by antibody staining, the separated proteins on the gel had to be transferred to a nitrocellulose membrane by Western blotting. The nitrocellulose membrane was equilibrated in transfer buffer (25 mM Tris, $192 \mathrm{mM}$ glycine, 20\% methanol). Membrane and blotting paper were well soaked with transfer buffer before arranging them as follows on the semidry blot chamber: 3 filter papers, membrane, gel, 3 filter papers.

The blotting was performed in transfer buffer at $2 \mathrm{~mA} / \mathrm{cm}^{2}$ for $60 \mathrm{~min}$. The efficiency of the transfer was determined by Ponceau-S Staining. Subsequently, the membrane was blocked with 5\% milk powder in PBS for $1 \mathrm{~h}$ at RT. Staining with the primary antibody was done by incubating the membrane in an antibody dilution overnight at $4{ }^{\circ} \mathrm{C}$. The membrane was washed three times for $15 \mathrm{~min}$ in PBS and in the following incubated with the secondary antibody for $1 \mathrm{~h}$ at RT. The primary antibody is detected by horseradish peroxidase (HRP) conjugated secondary antibodies that target the Fc portion of the primary antibody. After washing the membrane 3 times in PBS for $15 \mathrm{~min}$, the membrane was incubated with selfmade ECL (100 mM Tris-HCl, pH 8.5, $2.65 \mathrm{mM} \mathrm{H} \mathrm{H}_{2}, 0.45 \mathrm{mM}$ luminol, and $0.625 \mathrm{mM}$ coumaric acid) for $1 \mathrm{~min}$ or commercially available ECL for $5 \mathrm{~min}$. Chemiluminescence detection was performed by using chemiluminescence sensitive films or the LAS3000 system.

\subsection{Statistics}

All the statistical evaluations were performed using Graphpad Prism 4 software. Statistical analysis was performed using unpaired Student`s two-tailed t-test. Data are shown as means \pm SEM. Values of $\mathrm{p}<0.05(* \mathrm{p}<0.05)$ were considered statistically significant. 


\section{Results}

The role of PHD2 in macrophages has not been completely elucidated yet. In this project, two different models were used to investigate the effect of PHD2 deficiency in macrophages, i.e. BMDM and RAW cells. RAW cells are easy to cultivate and to genetically manipulate. BMDMs on the other hand are primary cells and thus resemble the in vivo situation better than the cell line. Thus, to gain insight into the functional consequences of an inhibition of PHD2, stably transduced RAW cells expressing constitutively a shRNA targeting PHD2 and BMDM isolated from wt or LysM Cre $\mathrm{x}$ PHD $2^{\text {fl/fl }}$ mice were used. Primary BMDM isolated from LysM Cre $\mathrm{x}$ PHD2 $2^{\mathrm{fl} / \mathrm{fl}}$ had been characterized in the literature before. However, metabolic alterations have not been analyzed in these cells. Since hypoxic adaptation is accompanied by changes in the cellular metabolism, the metabolic phenotype of PHD2 deficient macrophages was determined. Furthermore, I also analyzed if these change would alter the functionality of PHD2 deficient macrophages and how this is linked to the metabolic alterations at the molecular level.

\subsection{PHD2 deficient macrophages stabilize HIF-1 $\alpha$ and show a HIF target gene expression pattern in normoxia}

The experiments in this study were performed using BMDM isolated from PHD2 cKO mice and PHD2 knockdown RAW cells (shPHD2). The knockout and the knockdown of PHD2 respectively were confirmed on protein level (Fig. 6). To this end, wt and shPHD2 RAW cells as well as BMDM wt and PHD2 cKO macrophages were cultivated in normoxic $\left(20 \% \mathrm{O}_{2}\right)$ and hypoxic $\left(1 \% \mathrm{O}_{2}\right)$ conditions for $24 \mathrm{~h}$. Subsequently, cells were lysed and lysates were analyzed by Western blotting. PHD2 protein levels were significantly decreased in both PHD2 deficient macrophage cell models. The decrease in PHD2 protein levels could be observed under normoxic as well as hypoxic conditions. Whereas in the wt cells PHD2 protein levels increased in hypoxia in line with the fact that PHD2 per se is a HIF target gene, no comparable effect was observed in the shPHD2 and cKO cells. As a consequence of an efficient knockdown of PHD2, HIF-1 $\alpha$ protein levels are expected to increase since its degradation is inhibited. HIF-1 $\alpha$ levels were indeed increased in the shPHD2 RAW cells and cKO cells compared to wt cells in normoxia. In contrast, in wt BMDMs and RAW cells HIF- 
$1 \alpha$ protein levels were detected at low levels in normoxia and levels increased significantly only in hypoxic conditions. Taken together these experiments demonstrate that the PHD2 deficient RAW cell line model and the cKO BMDMs used in this project exhibit a biologically relevant reduction of PHD2 with subsequent stabilization of the HIF-1 $\alpha$ protein.

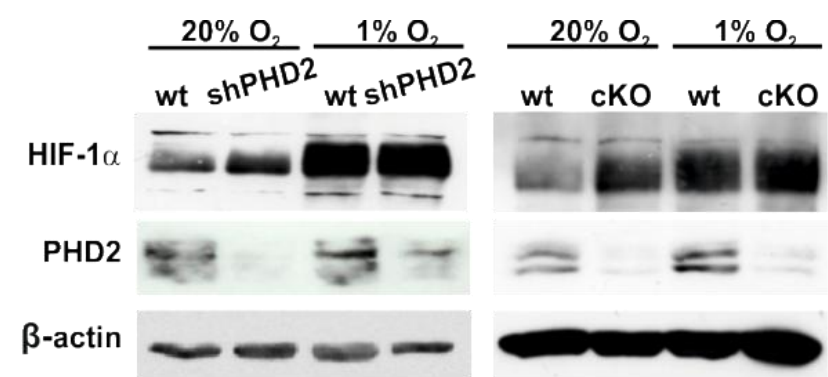

Figure 6: PHD2 protein levels are downregulated in shPHD2 cell and PHD2 cKO BMDMs. wt and shPHD2 RAW cells as well as BMDM wt and cKO macrophages were cultivated in normoxia $\left(20 \% \mathrm{O}_{2}\right)$ or hypoxia $\left(1 \% \mathrm{O}_{2}\right)$ for $24 \mathrm{~h}$. Subsequently cells were lysed and lysates were analyzed for HIF-1 $\alpha$, PHD2 and $\beta$-actin protein levels by Western blotting.

PHD2 is described to be the main regulator of HIF $\alpha$ stability in normoxia. Upon stabilization of the HIF $\alpha$ subunit and heterodimerization with the constitutively expressed HIF $\beta$ subunit, the transcription factor HIF-1 induces the expression of its target genes. Since the above described Western blot experiments indicated that HIF-1 $\alpha$ was sufficiently stabilized in the PHD2 deficient shPHD2 and cKO BMDMs in normoxia, I next analyzed how this effect is translated into altered HIF target gene expression. Therefore, I examined the mRNA expression levels of HIF target genes (including PHD2) in PHD2 cKO BMDM and shPHD2 cells in normoxia and hypoxia (Fig. 7). PHD2 cKO BMDM and shPHD2 RAW cells showed an $80 \%$ reduction of PHD2 mRNA compared to wt BMDM and wt RAW cells, which is in line with the observed decreased PHD2 protein levels. Among the known HIF target genes, especially metabolism-related target genes were selected for the RNA analyses namely GLUT-1, PFK1, PDK1, COX4-2, LonP and BNIP3. All of these target genes were upregulated in hypoxia in the wt cells indicating that the used experimental setup enables to demonstrate the cellular hypoxic response. In the shPHD2 RAW cells and PHD2 cKO BMDM, the mRNA expression of GLUT-1, PFK1, PDK1, COX4-2, LonP and BNIP3 mRNA was increased in normoxic conditions compared to the respective wt cells, which reflects the constitutive activation of the HIF system in these cells in normoxia. PHD3 mRNA expression is highly induced in hypoxia, which could also be seen in the wt cells in this study. Comparable to the metabolism-related HIF target genes PHD3 mRNA levels were significantly increased in the shPHD2 RAW and PHD2 cKO cells in normoxia compared to 
the wt cells. Most interestingly, however, the levels did not match the strong hypoxic induction of PHD3 mRNA levels in the wt cells indicating that the hypoxic gene expression pattern in the PHD2 deficient cell models just partly mimicked the response of the wt cells in hypoxia.

While HIF-1 $\alpha$ protein levels are significantly affected at the posttranslational level in hypoxia, HIF-1 $\alpha$ mRNA levels do not respond to hypoxia (Wenger et al., 1997). This was also the case in the cell models used in this study. mRNA levels of HIF-1 $\alpha$ were not affected by PHD2 deficiency or exposure to hypoxia. Taken together, the gene expression pattern of the PHD2 cKO and shPHD2 RAW cells resembled the induction of HIF target genes in wt BMDMs and wt RAW cells after incubation in hypoxia with the exception of PHD3 which was much stronger induced under hypoxic conditions.
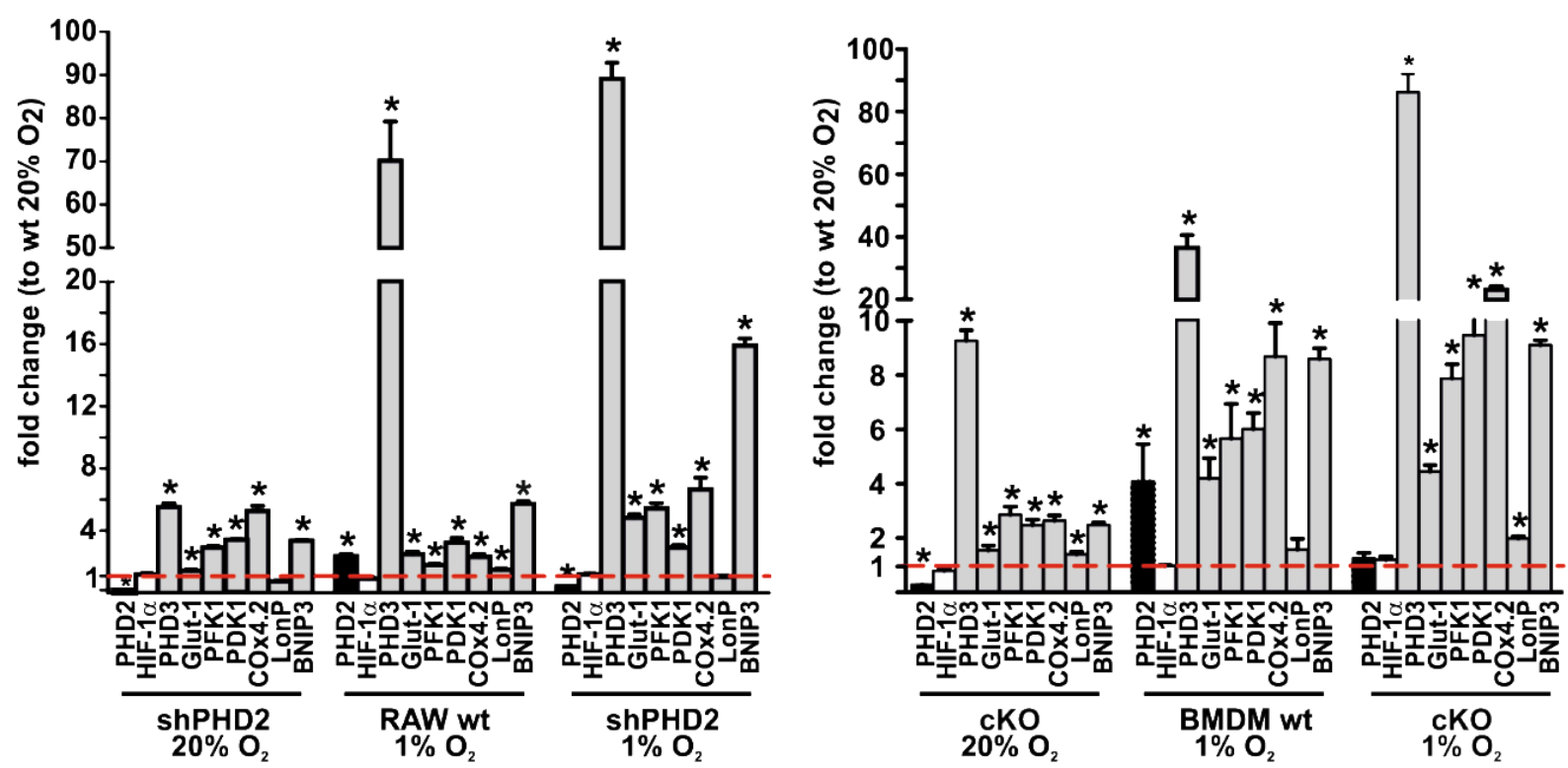

Figure 7: PHD2 deficient RAW and BMDM macrophages show an induction of HIF target genes in normoxic conditions. RAW wt and shPHD2 knockdown and BMDM wt and PHD2 cKO macrophages were incubated for $24 \mathrm{~h}$ at $20 \%$ or $1 \% \mathrm{O}_{2}$. Subsequently cells were lysed and mRNA was isolated. mRNA levels of HIF target genes and HIF-1 $\alpha$ itself were analyzed by qRT-PCR. The mRNA levels of RAW wt and BMDM wt cells (in normoxia) were set to one. The mRNA levels of shPHD2 or the PHD2 cKO BMDM or wt cells in hypoxia of the indicated genes are displayed as fold change compared to the wt cells in normoxia. $n=3-6$ samples per condition. mean \pm SEM, $* \mathrm{p}<0.05$

The target gene expression in the PHD2 deficient macrophages observed in normoxia is most likely mediated via HIF-1 $\alpha$. To test for this assumption, the mRNA levels of PHD2/HIF-1 $\alpha$ cKO BMDMs and the respective wt control cells were analyzed in normoxia and hypoxia (Fig. 8). Similar to the PHD2 cKO BMDMs, the double deficient (PHD2/HIF-1 $\alpha$ cKO) BMDMs demonstrated a strong reduction in PHD2 mRNA levels, which was accompanied by 
a significant decrease of HIF-1 $\alpha$ mRNA levels. In stark contrast to the above described effect seen in the PHD2 cKO BMDM, none of the analyzed HIF target genes was significantly increased in normoxic conditions. This demonstrates that indeed the hypoxic expression pattern of the HIF target genes seen in the PHD2 cKO BMDMs in normoxia is at least mainly mediated via HIF-1 $\alpha$.

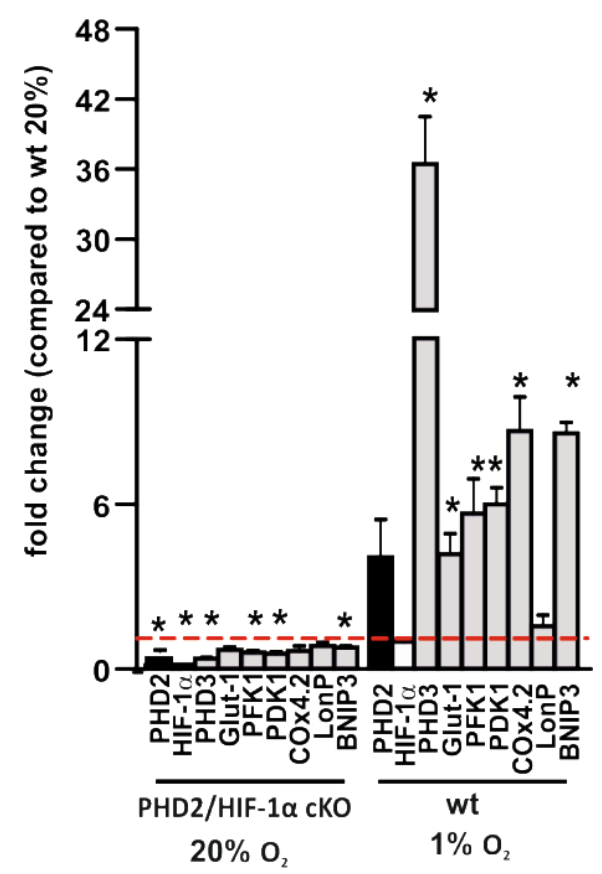

Figure 8: PHD2/HIF-1 $\alpha$ cKO BMDMs have unchanged or downregulated gene expression levels of HIF target genes in normoxic conditions. BMDM wt and PHD2/HIF-1 $\alpha$ cKO macrophages were incubated for $24 \mathrm{~h}$ at $20 \%$ or $1 \% \mathrm{O}_{2}$. The RNA expression levels of the indicated genes were analyzed by qRT-PCR. The RNA levels of BMDM wt cells were set to one. The RNA levels of PHD2/HIF-1 $\alpha$ cKO BMDM or wt cells in hypoxia of the indicated genes are displayed as fold change compared to the wt cells in normoxia. $\mathrm{n}=3$ samples per condition. mean $\pm \mathrm{SEM}, * \mathrm{p}<0.05$ 


\subsection{Migration and phagocytosis capacity is altered in PHD cKO and shPHD2 cells.}

\subsubsection{PHD2 deficient macrophages migrate less than wt macrophages}

Macrophages are motile cells and need to be able to migrate into the surrounding tissue. To obtain insight into the effects of PHD2 deficiency on macrophage physiology, the migratory capacity of PHD2 deficient macrophages was assessed using two different experimental set ups. Transmigration capacity was investigated using a Boyden chamber experimental setup. In addition to that, single cell migration capacity was assessed by determining the accumulated distance of migrated cells.

In the Boyden chamber experimental set up, wt and shPHD2 RAW as well as wt BMDM and BMDM cKO macrophages were confronted with either FCS-containing medium or with the conditioned supernatant of MDA-MB 231 breast carcinoma cells (Fig. 9 A). Migration was increased in the RAW cells and BMDM after stimulation with MDA-MB 231-conditioned supernatant. PHD2 cKO and shPHD2 cells were however found to migrate less compared to their respective wt cells in the transwell migration assay. This effect was seen after stimulating the cells with FCS and was even more prominent after stimulation with the conditioned MDA-MB 231 supernatant. Whereas in Boyden chamber experiment a mixture of cell invasion and migration is analyzed, single cell migration experiments allow analyzing the migration capacity per se. Therefore, the accumulated distance in single cell experiments over a time course of $6 \mathrm{~h}$ was analyzed (Fig. $9 \mathrm{~B}$ ). The cells were either stimulated with FCS or conditioned MDA-MB 231 supernatant. The accumulated migration distance of shPHD2 RAW cells and cKO BMDM was significantly reduced compared to the wt cells after stimulation with FCS as well as after stimulation with conditioned MDA-MB 231 supernatant. 
A

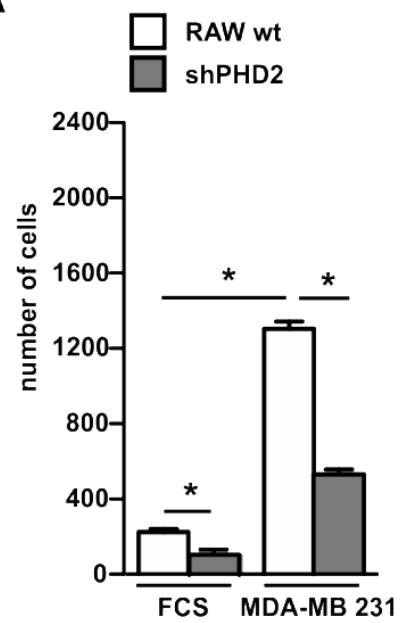

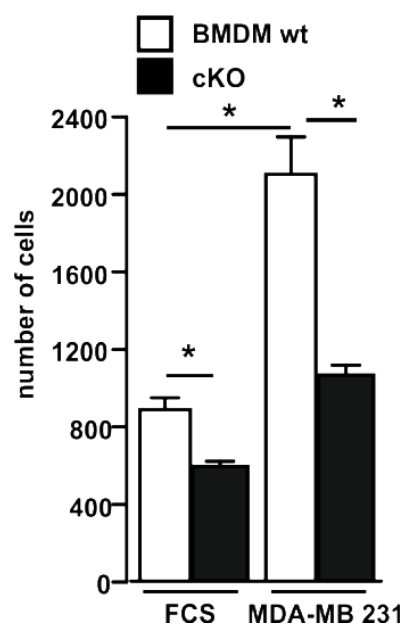
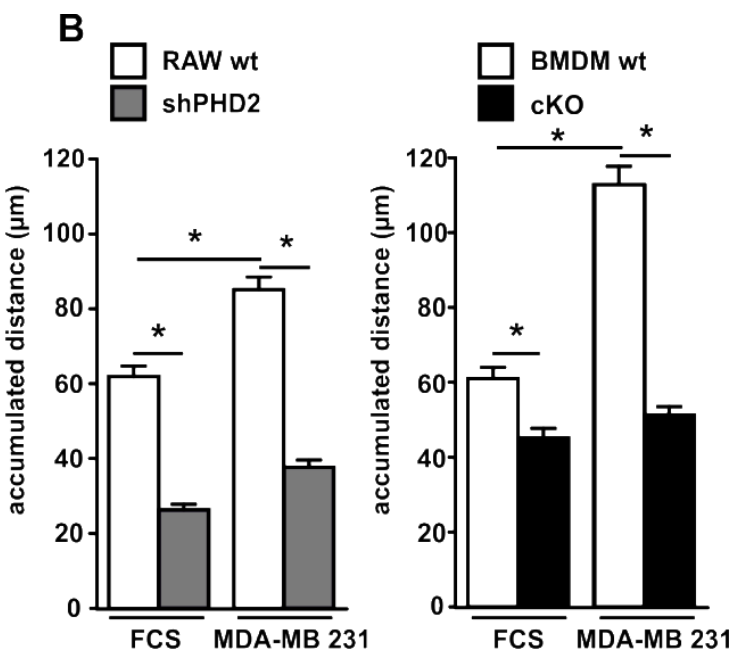

Figure 9: A reduction of PHD2 expression in RAW cells or BMDMs results in a defect in macrophage migration. (A) RAW wt, RAW shPHD2, BMDM wt and PHD2 cKO cells were tested for their migration capacity in Boyden chambers using fetal calf serum (FCS) or conditioned medium of MDA-MB 231 cells as a stimulus. $n=3$ (RAW cells), $n=4$ (BMDM) (B) The accumulated migration distance over $6 \mathrm{~h}$ of RAW wt, shPHD2 RAW, BMDM wt and PHD2 cKO cells was tested in single cell migration experiments using FCS or conditioned medium of MDA-MB 231 cells as stimulus. Bright field images were taken every $10 \mathrm{~min}$ over a period of $6 \mathrm{~h}$. Migration was analyzed using the cell tracking software Image J, $\mathrm{n}=59-64$ cells per condition (RAW cells), $\mathrm{n}=51-71$ cells per condition (BMDM cells). Graphs show one representative experiment of a set of 4-5 (Boyden chamber) and 3 (single cell migration) experiments. mean \pm SEM, $* \mathrm{p}<0.05$

In addition to measuring the migration of PHD2 deficient RAW cells and BMDMs, the migratory behavior of PHD2 cKO peritoneal macrophages was investigated. BMDMs are isolated from the bone marrow and differentiated over a time course of 7 days in vitro. In contrast, peritoneal macrophages are fully differentiated at the time of isolation and therefore even closer resemble the in vivo situation. Single cell migration experiments were performed with peritoneal macrophages isolated from PHD2 $\mathrm{cKO}$ mice and wt siblings. Freshly isolated peritoneal macrophages were subsequently tested for their migration capacity after stimulation with FCS or MDA-MB 231 conditioned medium. Comparable to the results obtained with the RAW cells and the BMDM migration of PHD2 cKO peritoneal macrophages was reduced compared to wt macrophages in both FCS and MDA-MB 231conditioned medium (Fig. 10). 


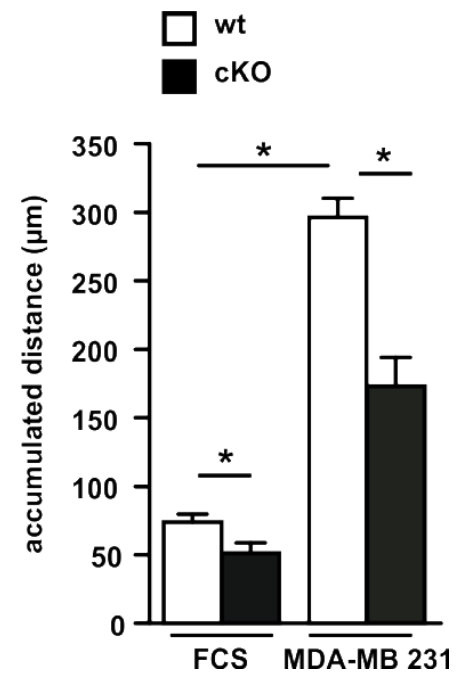

Figure 10: Peritoneal macrophages isolated from PHD cKO mice results in a defect in cell migration. The accumulated migration distance over $6 \mathrm{~h}$ of wt and PHD2 cKO peritoneal macrophages was tested in single cell migration experiments using FCS or MDA-MB 231 cell-conditioned medium as a stimulus. Bright field images were taken every 10 min over a period of 6 hours. Migration was analyzed using the cell tracking software ImageJ, n $=45-69$ cells per condition. Graphs show one representative experiment of a set of 3 experiments. mean \pm SEM, $* \mathrm{p}<0.05$

Deficiency of PHD2 in RAW cells or BMDMs led to the stabilization of HIF-1 $\alpha$ in normoxic conditions. A similar effect can be obtained by chemical inhibition of PHD activity in wt cells. PHD enzymes use $\alpha$-ketoglutarate as substrate during the hydroxylation reaction. Competitive inhibition of the $\alpha$-ketoglutarate binding results in inhibition of the enzymatic activity. DMOG is a potent $\alpha$-ketoglutarate analogue and is proposed to act as a competitive inhibitor against $\alpha$-ketoglutarate dependent dioxygenases, such as PHDs. Similar to the genetic deletion of PHD2, DMOG treatment results in HIF-1 $\alpha$ stabilization in normoxia. Thus, inhibition also the two other PHD isoforms would also be inhibited with DMOG treatment. It was tested, if pharmacological inhibition of PHD activity with DMOG affects single cell migration capacity of RAW wt cells (Fig. 11). The accumulated distance was found to be significantly decreased after stimulating the cells either with FCS or MDA-MB 231 conditioned cell culture medium. Besides $\alpha$-ketoglutarate, oxygen is the natural endogenous substrate of the PHD-mediated hydroxylation reaction. Hence, a reduction of the available oxygen concentration should result in a comparable effect as the treatment with DMOG. To test this hypothesis wt RAW cells were exposed to normoxic $\left(20 \% \mathrm{O}_{2}\right)$ or hypoxic $\left(1 \% \mathrm{O}_{2}\right)$ conditions. Single cell migration was tested under both conditions. Similar to the DMOG treated cells, hypoxic conditions significantly reduced the accumulated distance of the RAW wt cells. 


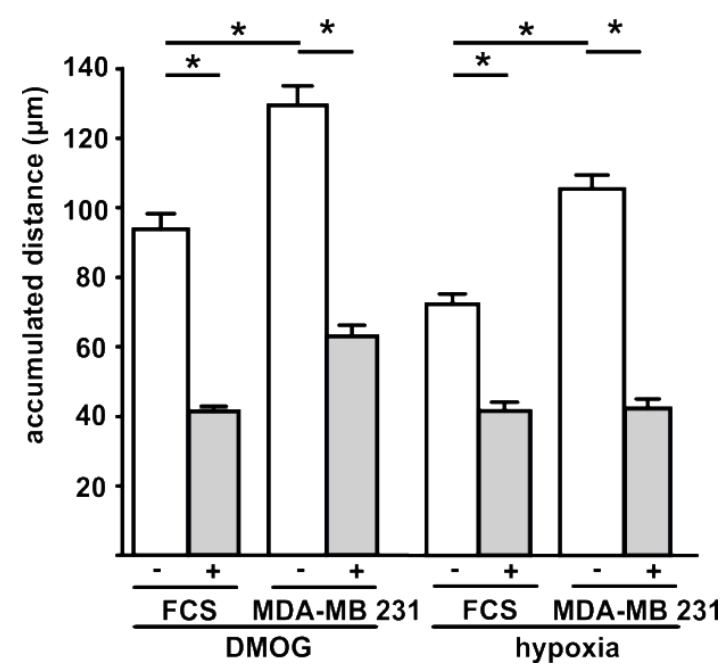

Figure 11: Migration of RAW wt cells is affected by DMOG and hypoxia exposure. RAW wt cells were incubated at $20 \% \mathrm{O}_{2} \pm 1 \mathrm{mM}$ DMOG or $1 \% \mathrm{O}_{2}$ for $6 \mathrm{~h}$. The accumulated migration distance was tested in single cell migration experiments using FCS or conditioned medium of MDA-MB 231 cells as stimulants. For cells analyzed in hypoxia, the hypoxic conditions were maintained during the single cell migration experiments without reoxygenation. $\mathrm{n}=50-53$ cells per condition. Graph shows one representative experiment out of a set of 2-3 experiments mean \pm SEM, * $\mathrm{p}<0.05$

Taken together, a reduction of PHD2 protein expression via a shRNA targeting PHD2 (RAW cells) or via a Cre LoxP system (BMDM) results in an impaired migration capacity of macrophages. Similar effects can be observed after chemical inhibition of PHD activity via treatment of wt macrophages with the $\alpha$-ketoglutarate analogue DMOG or by exposing the cells to hypoxic conditions.

\subsubsection{Phagocytosis is impaired in ShPHD2 RAW and PHD2 cKO macrophages}

After having reached the site of inflammation, macrophages phagocytose invading pathogens and cell debris. Therefore, phagocytosis is regarded as another good indicator for macrophage function besides migration. Phagocytosis can be determined by the uptake of fluorescently labeled beads. Fluorescence intensity of the cells reflects the uptake of the particles in these experiments. Similar to the experimental design described above, phagocytosis capacity was tested in the wt RAW, shPHD2, wt BMDM, and cKO cells (Fig. 12). Cells were either exposed to normoxic $\left(20 \% \mathrm{O}_{2}\right)$ conditions, hypoxic $\left(1 \% \mathrm{O}_{2}\right)$ conditions or treated with DMOG. Phagocytosis capacity was found to be significantly reduced in the shPHD2 and cKO cells compared to the wt cells. Exposing the wt macrophages to hypoxia or treatment with DMOG mimicked this effect observed in PHD2 deficient macrophages in the wt cells. 
$\square$ wt RAW

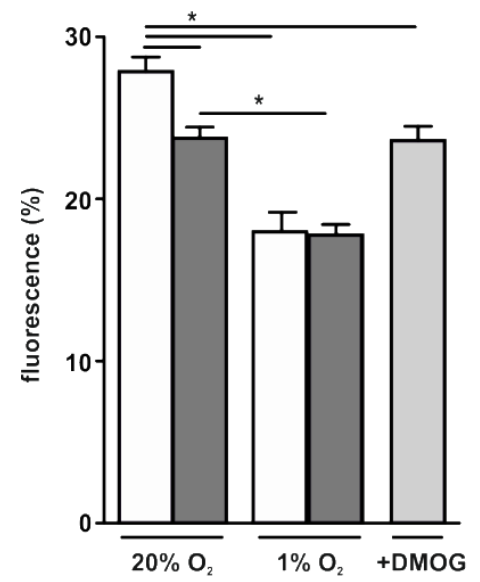

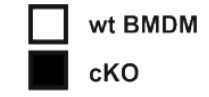

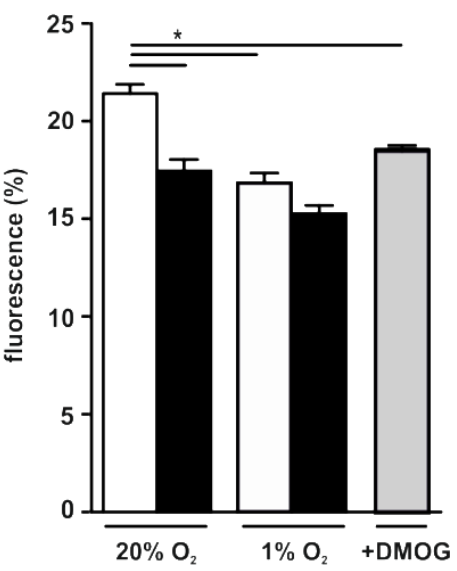

Figure 12: PHD2 deficient macrophages show a defect in phagocytosis. RAW wt, ShPHD2 RAW, BMDM wt and PHD2 cKO cells were incubated at $20 \% \mathrm{O}_{2} \pm 1 \mathrm{mM}$ DMOG or $1 \% \mathrm{O}_{2}$ in total for $20 \mathrm{~h}$. Then, fluorescently labeled beads were administered to the cells for another $4 \mathrm{~h}$ without reoxygenation. The capacity of the cells to phagocytose labeled beads was then analyzed. $\mathrm{n}=5$ samples per condition. Graphs show one representative experiment of a set of 3 experiments. mean \pm $\mathrm{SEM}, * \mathrm{p}<0.05$.

\subsubsection{Cell proliferation is not affected by PHD2-deficiency in RAW cells}

In principle resident macrophages are capable of self-renewal by local proliferation. In contrast, activated macrophages in contrast most commonly die during their course of action. Cell proliferation is an energy-consuming process and relies on the provision of sufficient ATP similar to migration and phagocytosis. Cell proliferation of RAW wt and shPHD2 macrophages was determined over 7 days (Fig. 13). To this end cells were seeded with a defined cell number and cell numbers were counted every day. Based on the cell counts, the doubling time was determined. Even though the cell numbers were slightly decreased in shPHD2 RAW cells at day 3 and 5 of the assay, in general there was not a significant difference between wt and shPHD2 cells. This is also reflected by the fact that the doubling time of the two cell lines is not significantly different. 

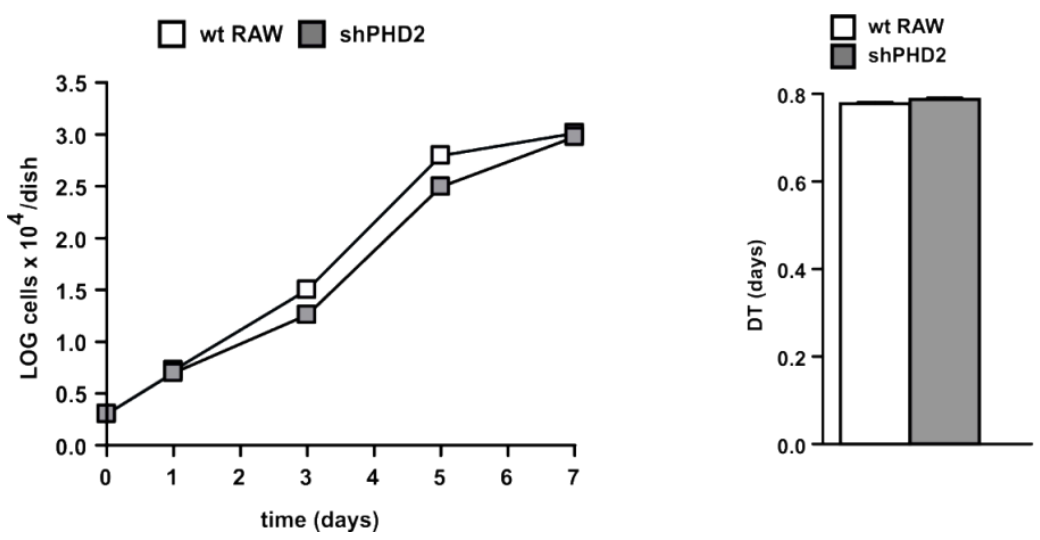

Figure 13: Cell proliferation is unchanged in wt and shPHD2 RAW macrophages. Cells were seeded at a concentration of $2 \times 10^{4}$ cells per dish and counted at the consecutive days for 7 days. Cell number is given as the logarithm of the cell number. Additionally, the doubling time (DT) was calculated based on the cell counts. $n=3$ per condition. Graphs show one representative experiment out of a set of 2 experiments. mean \pm SEM, $* \mathrm{p}<0.05$.

\subsection{PHD2 deficient macrophages have an altered metabolism}

\subsubsection{ATP levels are reduced in PHD2 cKO and shPHD2 macrophages}

To maintain proper cell function, cells rely on a fully functional cellular metabolism to produce sufficient ATP and meet energy demands. Activated macrophages utilize mainly glycolytic ATP to maintain integrity. As several HIF target genes that were found to be upregulated in the PHD2 deficient macrophages (chapter 3.1), play a role in the regulation of energy metabolism, the effect of PHD2 deficiency on cellular ATP levels was investigated (Fig. 14). The source for ATP production might change in normoxic $\left(20 \% \mathrm{O}_{2}\right)$ compared to hypoxic $\left(1 \% \mathrm{O}_{2}\right)$ conditions. Therefore, ATP levels were investigated in cells cultured at both oxygen concentrations. Levels of ATP were significantly diminished in the PHD2 cKO BMDM and shPHD2 RAW cells in normoxia (Fig. 14 A). In wt cells, ATP levels were reduced in hypoxia to similar levels as observed in the cKO BMDM and shPHD2 RAW cells in normoxia. Interestingly, in the PHD2 deficient cells ATP levels were even further reduced after exposure to hypoxia. Inhibition of PHD activity via treatment with DMOG mimicked the effect seen in the shPHD2 and cKO cells in normoxia as well as the effect seen in wt cells in hypoxia (Fig. 14 A). 

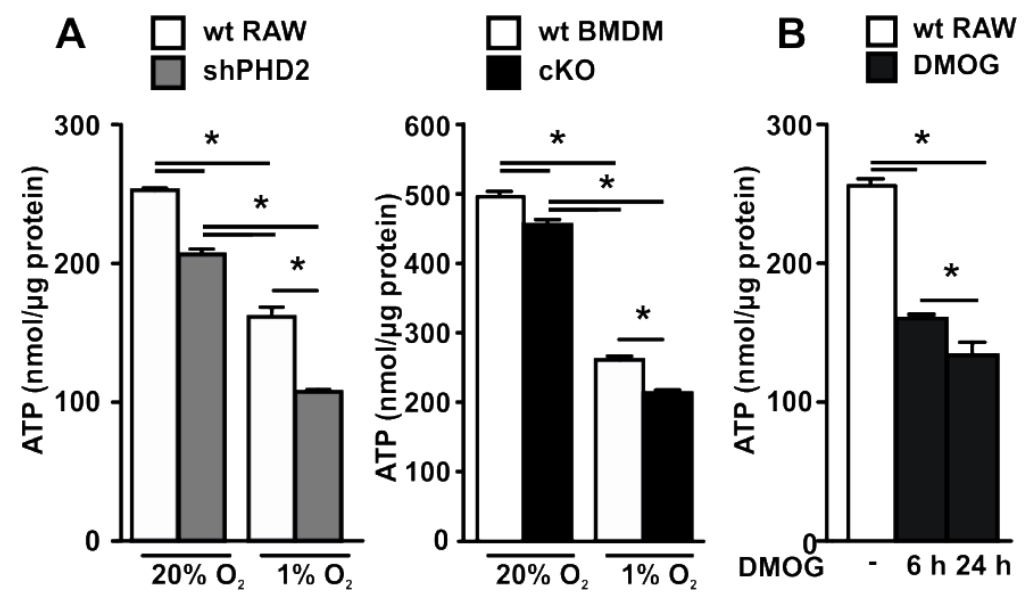

Figure 14: ATP levels are reduced in PHD2 deficient macrophages. (A) RAW wt, shPHD2 RAW, BMDM wt and PHD2 cKO cells were incubated at $20 \% \mathrm{O}_{2}$ or $1 \% \mathrm{O}_{2}$ for $24 \mathrm{~h}$. Intracellular ATP levels were determined. $\mathrm{n}=6$ samples per condition. (B) RAW wt cells were incubated for 6 and $24 \mathrm{~h}$ with $1 \mathrm{mM}$ DMOG. Subsequently intracellular ATP levels were determined. Graphs show one representative experiment of 5 (A) or 4 (B) sets of experiments. $n=6-7$ per condition. mean \pm $\mathrm{SEM}, * \mathrm{p}<0.05$.

\subsubsection{Oxygen consumption rate is altered in PHD2 cKO and shPHD2 cells}

The adaptation to hypoxic conditions involves a metabolism shift from aerobic to anaerobic glycolysis. This can be explained in part by the fact that mitochondrial respiration consumes oxygen which needs to be saved when oxygen availability is scarce. To gain insight into the metabolic profile of the wt and PHD2 deficient cells the oxygen consumption rate (OCR) was analyzed. The OCR was measured while interfering with mitochondrial respiration by addition of chemicals using a Seahorse XF96 analyzer (Fig. 15 A and B).

The basal OCR of the PHD2 cKO and the shPHD2 cells was significantly decreased compared to the respective wt. Adding Oligomycin to the cells blocks their ATP synthase. Therefore, oligomycin can be used to distinguish between the amount of oxygen consumption of the cells due to proton leak and the amount of oxygen consumption due to ATP synthesis. Addition of oligomycin indeed decreased the OCR. The effect of oligomycin was more severe in the wt compared to PHD2 deficient cells. This further supported the fact that mitochondrial ATP synthesis is reduced in PHD2 cKO and shPHD2 macrophages. Addition of FCCP allows analyzing the maximal respiration of the cells. The maximal respiration after uncoupling the mitochondria with the ionosphere FCCP was also significantly decreased in the PHD2 deficient cells compared to the respective wt cells. This shows that the capacity of the shPHD2 and PHD2 cKO macrophages to use the mitochondria for respiration is reduced, thus the maximal respiratory capacity is decreased. Inhibition of complex I and III by the inhibitors 
rotenone and Antimycin A blocks mitochondrial respiration. This then shows the residual OCR, which is not due to mitochondrial respiration. In the PHD2 cKO as well as the shPHD2 cells, this residual respiration was still decreased compared to the respective wt cells. This indicates that not only mitochondrial OCR, but also other oxygen-consuming pathways are affected by PHD2 deficiency.

Taken together, basal and maximal respiration are significantly affected as a consequence of decreased PHD2 levels in RAW cells and BMDMs. This finding strongly indicates that aerobic glycolysis is reduced in these cells due to the switch to anaerobic glycolysis.
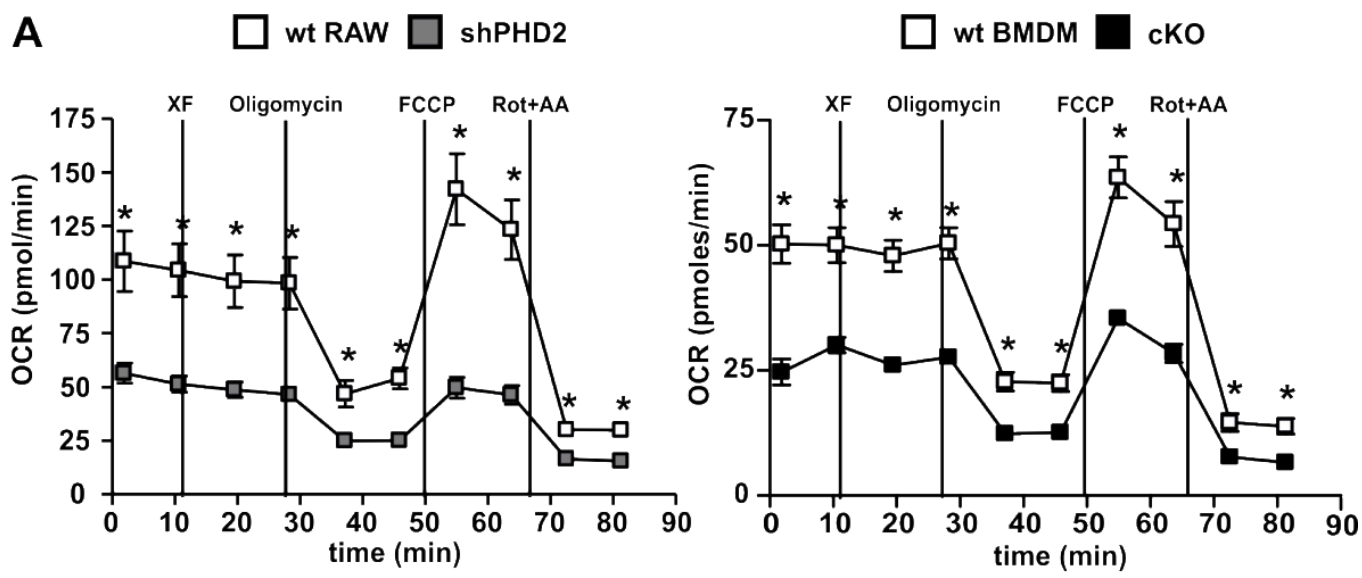

B
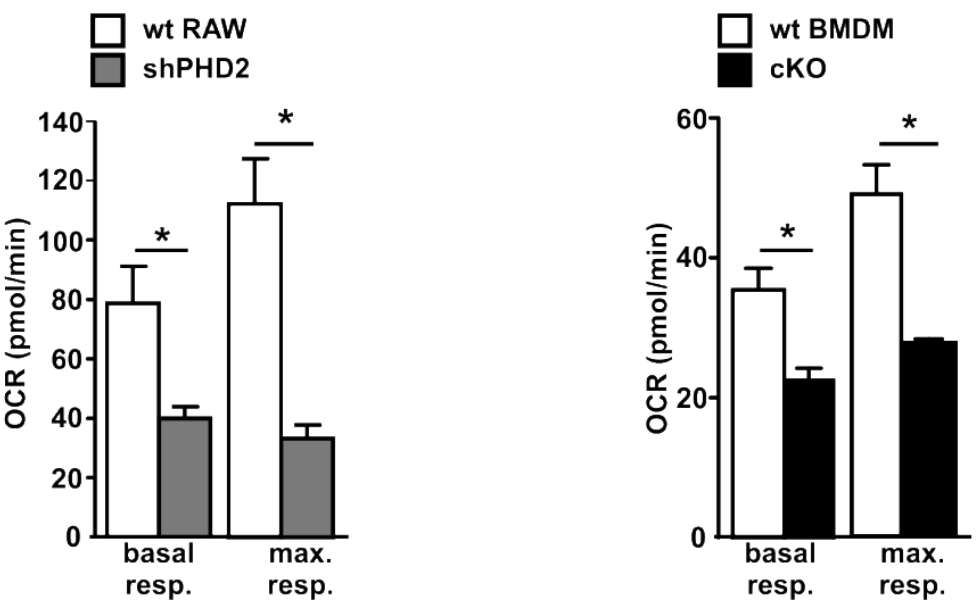

Figure 15: Oxygen consumption rate is decreased in PHD2 deficient macrophages. (A) RAW wt, shPHD2 RAW, BMDM wt and PHD2 cKO cells were tested for their oxygen consumption rate (OCR) after addition of XF-medium, oligomycin, FCCP as well as rotenone and antimycin A (Rot $+\mathrm{AA}) . \mathrm{n}=6$ (RAW) and $\mathrm{n}=10$ (BMDM) samples per condition. (B) Basal respiration and maximum respiratory capacity were analyzed in RAW wt, shPHD2 RAW, BMDM wt and PHD2 cKO cells based on the experiment shown in A. The OCR after Rot +AA addition was subtracted from the OCR after XF addition (basal respiration) and after FCCP uncoupling (maximal respiration). Graphs show one representative experiment of a set of 4-6 experiments. mean \pm SEM, $* \mathrm{p}<0.05$ 
OCR was also determined in RAW wt cells, which were treated with DMOG (Fig. 16). Mitochondrial oxygen consumption was significantly reduced in the treated cells. This was observed for the basal respiration, the respiration after oligomycin addition and the maximal respiratory capacity after uncoupling the mitochondria with FCCP. Taken together, this indicates that PHD2 deficient macrophages heavily reduce mitochondrial oxygen consumption and that this can be mimicked by pharmacological inhibition of the PHDs.
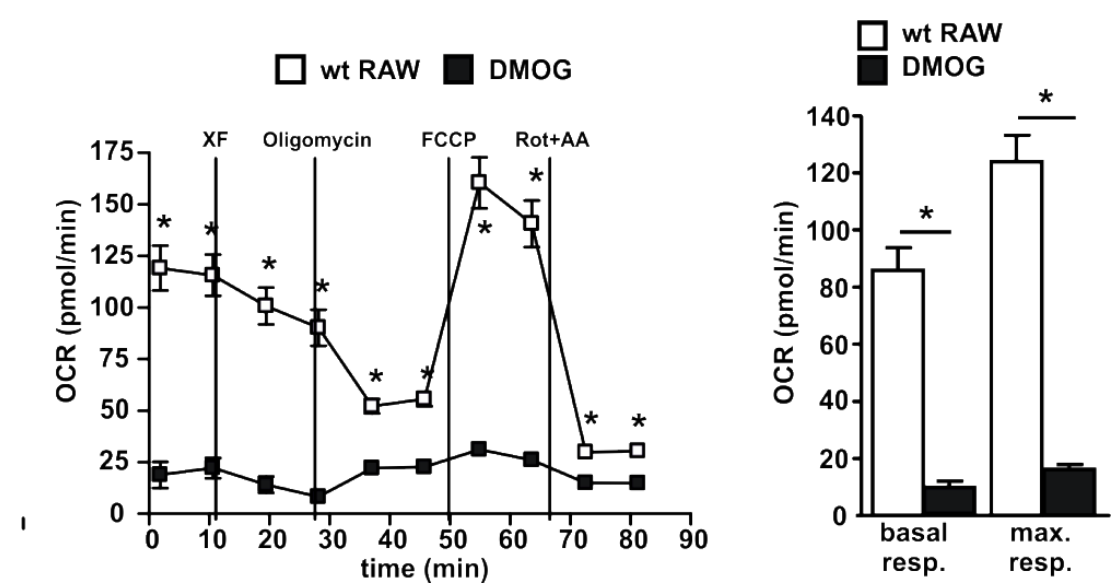

Figure 16: RAW wt cells treated with DMOG have depressed oxygen consumption rate. RAW wt cells treated with $1 \mathrm{mM}$ DMOG for $24 \mathrm{~h}$ were tested for their oxygen consumption rate (OCR) after addition of XF-medium, oligomycin, FCCP as well as rotenone and antimycin A (Rot $+\mathrm{AA}) \cdot \mathrm{n}=6$ samples per condition. Basal respiration and maximum respiration were analyzed. The OCR after Rot $+\mathrm{AA}$ addition was subtracted from the other rates. Graphs show one representative experiment of a set of 3 experiments. mean $\pm \mathrm{SEM}, * \mathrm{p}<0.05$

\subsubsection{Extracellular acidification rate is increased in PHD2 $\mathrm{cKO}$ and shPHD2 cells}

Anaerobic glycolysis for energy generation results in significantly higher rate of proton production compared to cells generating energy from oxidative phosphorylation. Therefore, the extracellular acidification rate (ECAR) indicates the energy source of the cell. To proof that as a consequence of a reduction of PHD2 macrophages switch to anaerobic glycolysis, the ECAR of wt, shPHD2 and cKO BMDM cells was analyzed (Fig. 17). In these experiments the ECAR was analyzed after the addition of glucose, which allows determining the glycolysis rate, and oligomycin, which indicates the glycolytic capacity.

PHD2 cKO as well as shPHD2 cells displayed an increased ECAR compared to their respective wt cells after the inhibition of mitochondrial oxidative phosphorylation by oligomycin. The shPHD2 RAW cells showed not only an increased glycolytic capacity but 
also the basal glycolysis measured after the addition of glucose was elevated. This effect was not observed in the shPHD2 cells.
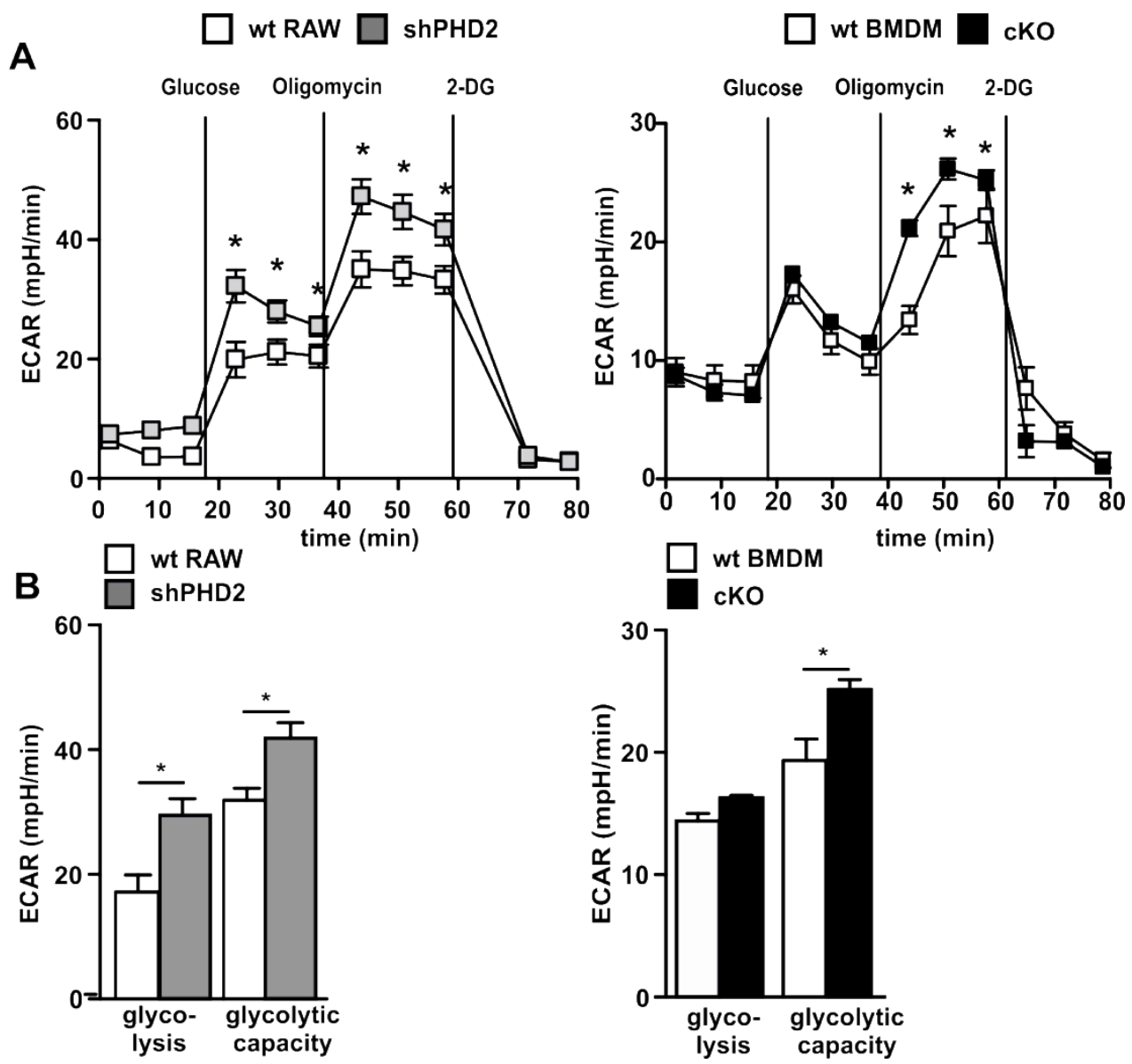

Figure 17: Extracellular acidification rate is increased in PHD2 deficient cells. (A) Extracellular acidification rate (ECAR) was determined in RAW wt, shPHD2 RAW, BMDM wt and PHD2 cKO cells or RAW wt cells treated with $1 \mathrm{mM}$ DMOG for $6 \mathrm{~h}$ after addition of glucose, oligomycin and 2-deoxy glucose (2-DG). $\mathrm{n}=6$ samples per condition. (B) Glycolysis and anaerobic glycolytic capacity were analyzed based on the experiments shown in A. The ECAR measured after 2-DG addition was subtracted from the ECAR after glucose (glycolysis) and oligomycin addition (glycolytic capacity). Graphs show one representative experiment out of a set of 3-4 experiments. mean \pm SEM, * $p<0.05$

\subsubsection{Lactate levels are increased in PHD2 deficient macrophages}

In anaerobic glycolysis, glucose is converted to lactate. An increase in glycolysis therefore should be accompanied by an increase in lactate levels in the supernatant of the cells. The above described OCR and ECAR assays indicate that the shPHD2 and cKO macrophages mostly use anaerobic glycolysis for energy production despite an abundant presence of oxygen. To support these findings, lactate levels were determined in the supernatants of wt, shPHD2 and cKO macrophages cultured in normoxic $\left(20 \% \mathrm{O}_{2}\right)$ or hypoxic $\left(1 \% \mathrm{O}_{2}\right)$ conditions (Fig. 18). In line with the reduced OCR and increased ECAR, lactate levels in the 
supernatants of PHD2 cKO and shPHD2 RAW cells were increased compared to their respective wt cells. Lactate levels were also increased after exposure of the cells to hypoxic conditions for $24 \mathrm{~h}$, which indicates that the experimental conditions used are adequate to determine the well-described switch to anaerobic glycolysis in hypoxia. Treatment of wt RAW cells with DMOG mimicked the increased lactate levels in hypoxia although in absolute terms the levels were lower and were more comparable to the levels found in the shPHD2 cells.
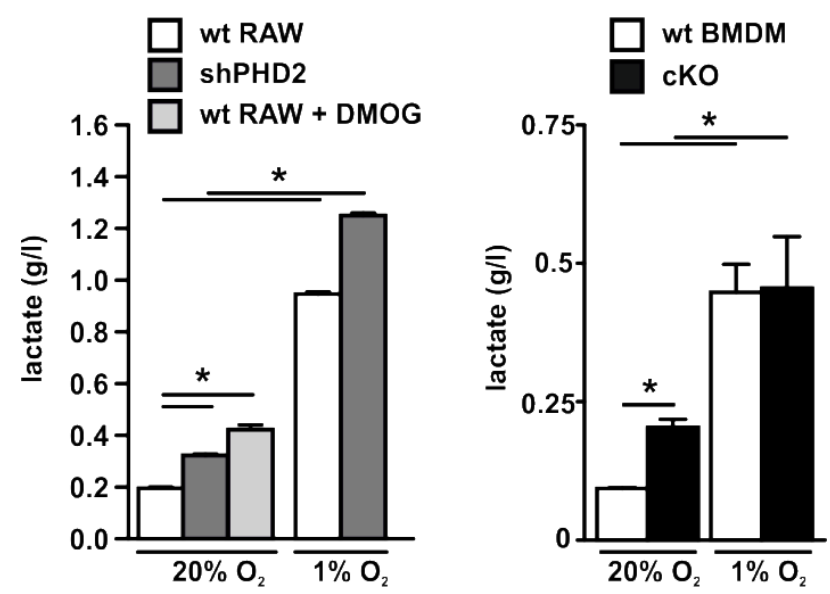

Figure 18: Lactate levels are increased in the supernatant of PHD2 cKO and shPHD2 RAW cells. Lactate levels were determined in the supernatant of RAW wt, shPHD2 RAW, BMDM wt and PHD2 cKO cells after incubation of the cells at the indicated conditions. $n=4$ samples per condition. Graphs show one representative experiment of a set of 4-6 experiments. mean \pm SEM, $* \mathrm{p}<0.05$

\subsubsection{Mitochondrial content of PHD2 deficient macrophages is unaltered}

A decrease in oxygen consumption accompanied by the increased ECAR and lactate levels as observed in the PHD2 deficient macrophages can result from various reasons. One possibility is as discussed in the previous chapter 3.3, a glycolytic switch in order save the available oxygen. Another possibility is that PHD2 deficiency affects the number of mitochondria per cell. To exclude that the observed phenotype is due to an altered mitochondrial number rather than a shift in cellular metabolism, the mitochondrial content was determined in the wt, shPHD2 and cKO macrophages. To this end the amount of the mitochondrial gene mitochondrial COX2 (mtCO2) was compared to the amount of the genomic DNA for the ribosomal subunit $18 \mathrm{~S}$ (Fig 19) by qPCR. This ratio indicates indirectly the number of mitochondria per cell. No difference in the mtCO2/18S was observed in the shPHD2 or wt 
RAW cells or in the PHD2 $\mathrm{cKO}$ and their respective wt. This indicates that the number of mitochondria is unaltered in the PHD2 deficient macrophages.
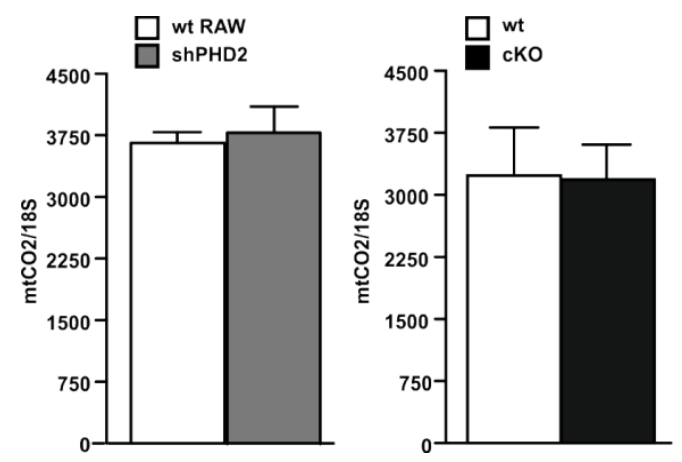

Figure 19: Mitochondrial content is unaltered in ShPHD2 and cKO macrophages compared to the respective wt cells. Mitochondrial content of wt and shPHD2 cells was analyzed by determining the ratio of the copy number for the mitochondrial gene mtCO2 and genomic DNA 18S. Graph shows one representative experiment out of a set of 2-3 experiments $\mathrm{n}=6$ samples per condition. mean $\pm \mathrm{SEM}, * \mathrm{p}<0.05$

\subsubsection{Inhibition of mitochondrial respiration decreased migration}

To summarize the findings described so far, PHD2 deficient macrophages display a significantly impaired migration and phagocytosis, which are associated with a decrease in ATP production and mitochondrial oxygen consumption. To allow the conclusion that decreased mitochondrial oxygen consumption indeed results in an altered migration, RAW wt cells were treated with the mitochondrial complex I inhibitor rotenone. Subsequently, single cell migration was analyzed using the treated versus non-treated cells by stimulating the cells with FCS or MDA-MB 231 conditioned medium (Fig. 20). The importance of mitochondrial function for migration capacity was further stressed by the fact that single cell migration of RAW wt cells was found to be decreased when treated with rotenone. 


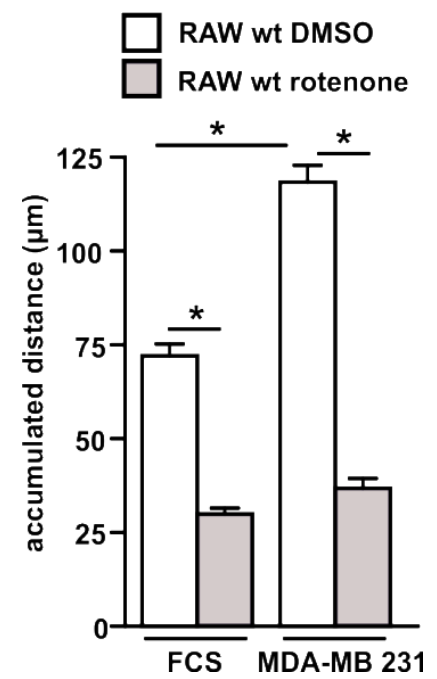

Figure 20: Rotenone treatment decreases migration of RAW wt cells in single cell migration experiments. The accumulated migration distance of RAW wt cells over $6 \mathrm{~h}$ after incubation of the cells $\pm 1 \mu \mathrm{M}$ rotenone or DMSO (used as diluent control) for $24 \mathrm{~h}$ was determined in single cell migration experiments using FCS or MDA-MB 231 conditioned medium as stimulus. $\mathrm{n}=51-53$ cells per condition. Graph shows one representative experiment out of a set of 3 experiments. mean $\pm \mathrm{SEM}, * \mathrm{p}<0.05$

\subsubsection{Mitochondrial ROS production is decreased in shPHD2 RAW cells}

In hypoxia, cells display a cytochrome c subunit switch. The cytochrome c subunit switch from COX4-1 to COX4-2 increases the efficiency of complex IV of the mitochondrial electron transport chain regarding the quantity of ATP and ROS produced (Fukuda et al., 2007). Furthermore, the HIF-mediated increase of the glycolytic flux reduces the flux through the TCA cycle and the mitochondrial electron transport chain. This way, the accumulation of mitochondria-derived ROS is reduced. As described in chapter 3.1, the analysis of the mRNA levels in the shPHD2 cells indicated a cytochrome subunit switch. Additionally, analysis of the OCR and ECAR indicated a glycolytic switch. To investigate if these changes are associated with changes in mitochondrial ROS levels, ROS production was analyzed using mitochondria isolated from wt and shPHD2 RAW cells (Fig. 21). ROS accumulation was analyzed by DCF staining. Using this method, ROS accumulation was found to be significantly reduced as a consequence of the PHD2 knockdown in isolated RAW mitochondria. 


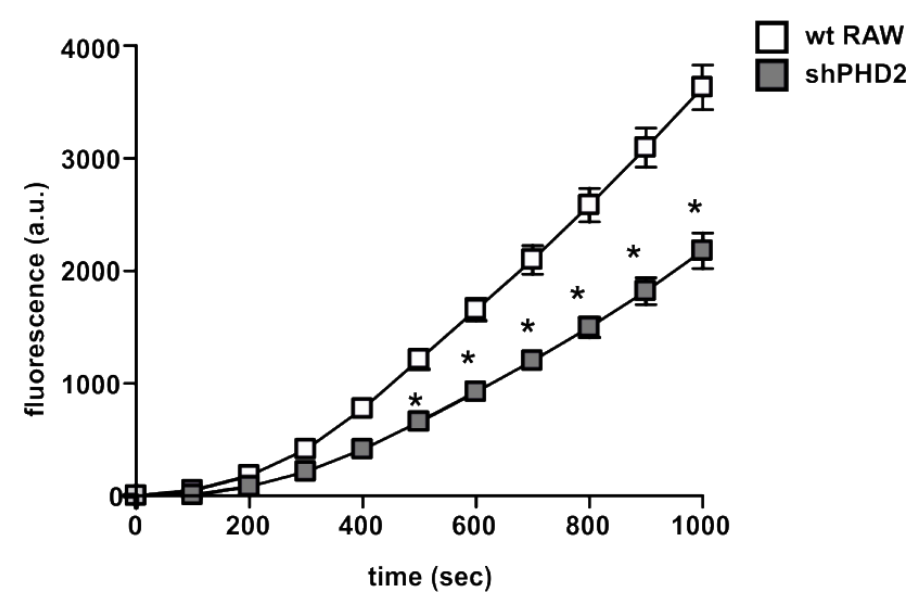

Figure 21: PHD2 affects mitochondrial ROS production. Mitochondria of wt and shPHD2 RAW cells were isolated and analyzed for ROS production by DCF staining. Graph shows one representative experiment of a set of 5 experiments mean \pm $\mathrm{SEM}, * \mathrm{p}<0.05$

\subsubsection{Overall ROS production is increased in ShPHD2 RAW cells}

Even though mitochondria are thought to be the main producers of cellular ROS, there are also many other mechanisms contributing to overall cellular ROS (reviewed in (Holmstrom and Finkel, 2014)). Total cellular ROS levels were analyzed by quantification of the EOH/DHE and the ethidium/DHE ratios in RAW wt and shPHD2 cells (Fig. 22). EOH/DHE indicates the amount of ROS due to superoxide $\left(\mathrm{O}_{2}{ }^{\circ}\right)$ production and the ethidium/DHE ratio indicates the amount of ROS due to $\mathrm{H}_{2} \mathrm{O}_{2}$ production and other ROS sources. Interestingly, while mitochondrial ROS production was reduced, the ROS production in complete cells was found to be increased. Both, i.e. the ratio for $\mathrm{EOH}$ indicating $\mathrm{O}_{2}{ }^{--}$and for Ethidium indicating $\mathrm{H}_{2} \mathrm{O}_{2}$, were found to be significantly increased in shPHD2 cells compared to wt cells. This shows that despite the decreased mitochondrial ROS production other ROS-producing pathways are increased in shPHD2 RAW cells. This results in a net increased ROS level in these cells. 


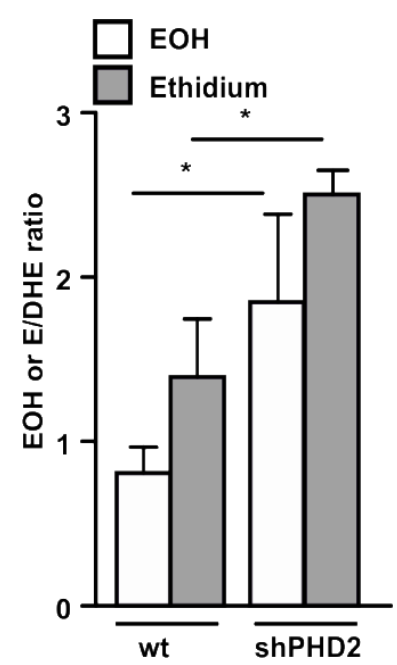

Figure 22: HPLC analysis of DHE derived products in whole cell lysates of wt and shPHD2 RAW cells. Quantification of EOH/DHE and ethidium/DHE ratios from RAW whole cell lysates. Cells were maintained in PBS and incubated with $100 \mu \mathrm{M}$ DHE for $30 \mathrm{~min}$ and analyzed by HPLC. Graph shows one representative experiment of a set of 3 experiments. $\mathrm{n}=3$ per condition. mean $\pm \mathrm{SEM}, * \mathrm{p}<0.05$

\subsection{The metabolic alterations observed in PHD2 deficient macrophages depend on PDK1}

\subsubsection{PDK1 protein levels are increased in PHD2 deficient macrophages}

PDK1 is an important factor mediating the switch from aerobic to anaerobic glycolysis (see chapter 1.3.4). PDK1 phosphorylates and thereby inhibits PDH which results in less pyruvate to be converted into acetyl-CoA. Pyruvate then is metabolized via the glycolytic pathway into lactate. As described in chapter 3.1, PHD2 cKO and shPHD2 cells had increased PDK1 RNA levels in normoxia, which was mediated via HIF-1 $\alpha$. To confirm this finding at the protein level, cell lysates of wt RAW, shPHD2, wt BMDM and cKO BMDM were prepared after culturing the cells for $24 \mathrm{~h}$ in normoxic $\left(20 \% \mathrm{O}_{2}\right)$ or hypoxic $\left(1 \% \mathrm{O}_{2}\right)$ conditions. Lysates were subsequently analyzed for PDK1 protein levels by Western blot analysis (Fig. 23). In all cells, PDK1 protein levels were significantly increased in hypoxia compared to normoxia. This is in line with the fact that PDK1 is a well characterized HIF target gene. In the PHD2 deficient RAW cells and cKO BMDM, however, elevated PDK1 levels were also detected in normoxia compared to the wt control cells. These protein levels were comparable to the levels found in hypoxia. Accordingly, PDH was found to be more phosphorylated in the PHD2 cKO and shPHD2 RAW cells compared to their respective wt cells in normoxia whereas the 
amount of total PDH remained unchanged. As a loading control, $\beta$-actin protein levels were analyzed.

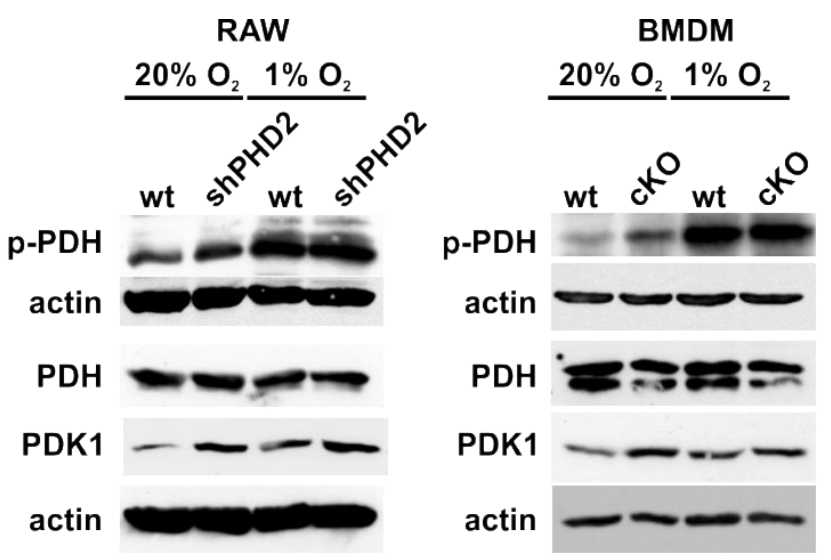

Figure 23: PDK1 protein levels are increased in PHD2 cKO and shPHD2 cells. Phospho-PDH, total PDH, PDK1 and $\beta$ actin protein levels of RAW wt and shPHD2 cells as well as BMDM wt and PHD2 cKO macrophages in normoxia $\left(20 \% \mathrm{O}_{2}\right)$ or hypoxia $\left(1 \% \mathrm{O}_{2}\right.$ for $\left.24 \mathrm{~h}\right)$.

\subsubsection{PHD2 deficient macrophages show a decreased activity of PDH}

PDK1 is the key enzyme for the glycolytic switch from aerobic to anaerobic metabolism. At the molecular level, this is reflected by enhanced phosphorylation of PDH. This posttranslational modification alters the activity of the pyruvate metabolizing enzyme. With increased phosphorylation the activity ceases, which prevents pyruvate to be fed into the TCA cycle. Since an increased phosphorylation of PDH was found in the shPHD2 and cKO macrophages, the activity of the enzyme was analyzed (Fig. 24). The activity of PDH was determined in cell extracts isolated from wt cells and shPHD2 RAW cells after exposure to normoxia $\left(20 \% \mathrm{O}_{2}\right)$ or hypoxia $\left(1 \% \mathrm{O}_{2}\right)$ for $24 \mathrm{~h}$. Additionally, wt cells were treated with DMOG, lysed and subsequently tested for PDH activity. PDH activity was significantly reduced in shPHD2 cells in normoxia compared to wt cells. The wt cells showed a reduced activity of PDH after DMOG treatment or after exposure to hypoxia. However, PDH activity in the shPHD2 cells was still significantly lower in hypoxia compared to wt cells. The PDH activity thus reflected the phosphorylation pattern seen in the Western blots as well as the PDK1 mRNA and protein levels. 


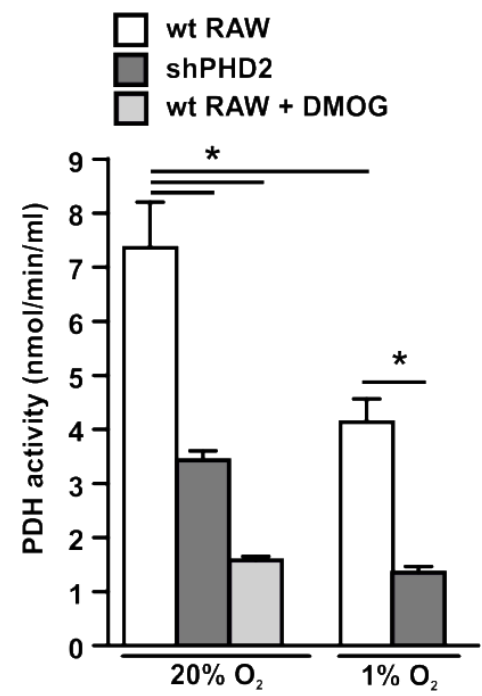

Figure 24: PDH activity is decreased in shPHD2 cells. PDH activity in RAW wt, shPHD2 RAW cells and RAW wt cells treated with $1 \mathrm{mM}$ DMOG for $24 \mathrm{~h}$ in normoxia or hypoxia $\left(1 \% \mathrm{O}_{2}\right.$ for $\left.24 \mathrm{~h}\right) . \mathrm{n}=4-6$ samples per condition. Graph shows one representative experiment of a set of 5 experiments. mean \pm SEM, $* \mathrm{p}<0.05$

\subsection{PDK inhibition by dichloroacetate (DCA) partly restores the metabolic alterations in PHD2 deficient macrophages}

\subsubsection{PDH activity in ShPHD2 cells is restored by dichloroacetate treatment}

From the data of the previous chapters, a downregulation of PHD2 was associated with an induction of HIF target genes, an impaired migration and phagocytosis as well as an altered metabolism. An important regulator of the metabolic shift to glycolysis, PDK1 was found to be increased. In line with this, PDH activity was found to be reduced in PHD2 deficient macrophages. In order to obtain further insight into the role of PDK1 in the observed phenotype, the cells were treated with the PDK inhibitor dichloroacetate (DCA). Binding of DCA to PDK1 triggers conformational changes resulting in the inactivation of kinase activity (Kato et al., 2007). To test if inhibition of PDK1 by DCA treatment indeed reestablished the PDH activity in the shPHD2 RAW cells, wt and shPHD2 RAW cells were treated for $24 \mathrm{~h}$ with $5 \mathrm{mM}$ DCA (Fig. 25). Subsequently, cells were lysed and analyzed for PDH activity. As demonstrated before in chapter 3.4.2, shPHD2 macrophages exhibited a significantly lower PDH activity compared to wt cells. In the DCA-treated shPHD2 cells however, an increase in PDH activity that partly resembles the activity observed in the wt, was detected. DCA did not have a significant effect on the wt cells. 


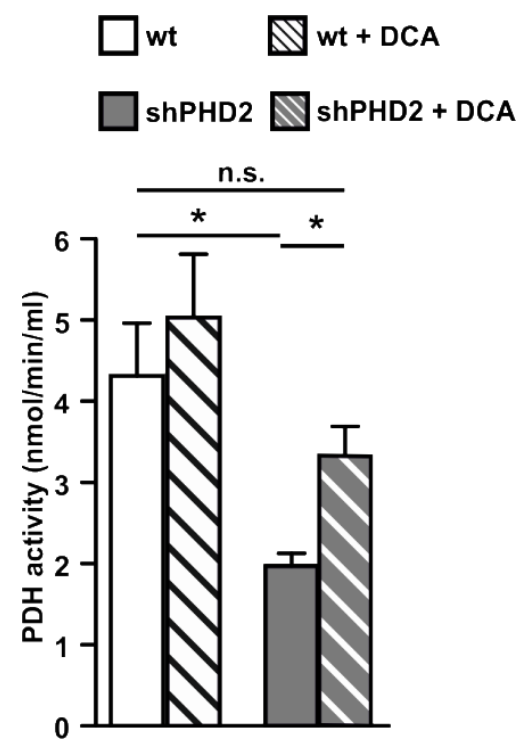

Figure 25: PDH activity is increased after treatment with dichloroacetate (DCA). PDH activity was determined in RAW wt and shPHD2 RAW cells after incubation of the cells $\pm 5 \mathrm{mM}$ DCA for $24 \mathrm{~h} . \mathrm{n}=6$ samples per condition. Graph shows one representative experiment of a set of 4 experiments. mean \pm SEM, $* \mathrm{p}<0.05$

\subsubsection{Lactate levels are decreased by DCA treatment}

The increase in PDH activity described above should result in a decrease in lactate levels as PDH converts pyruvate to acetyl CoA which should result in a decrease of anaerobic glycolysis and hence a reduction in the lactate levels produced. In line with the increased PDH activity, DCA treatment of PHD2 cKO and shPHD2 macrophages also affected lactate levels in these cells (Fig.26). The increase in lactate levels found in the untreated shPHD2 and PHD2 cKO macrophages could be decreased by treating them with $5 \mathrm{mM}$ DCA for $24 \mathrm{~h}$. Even though PDH activity was not found to be affected by DCA treatment in the wt cells, lactate levels were also reduced in the DCA treated wt. This could indicate that DCA also has a minor effect on PDH activity in the wt RAW cells which cannot be detected by this assay, but in an assay where an endpoint is measured, such as the accumulation of lactate. However, the effect on lactate levels seems to be stronger in the shPHD2 and PHD2 cKO macrophages. 

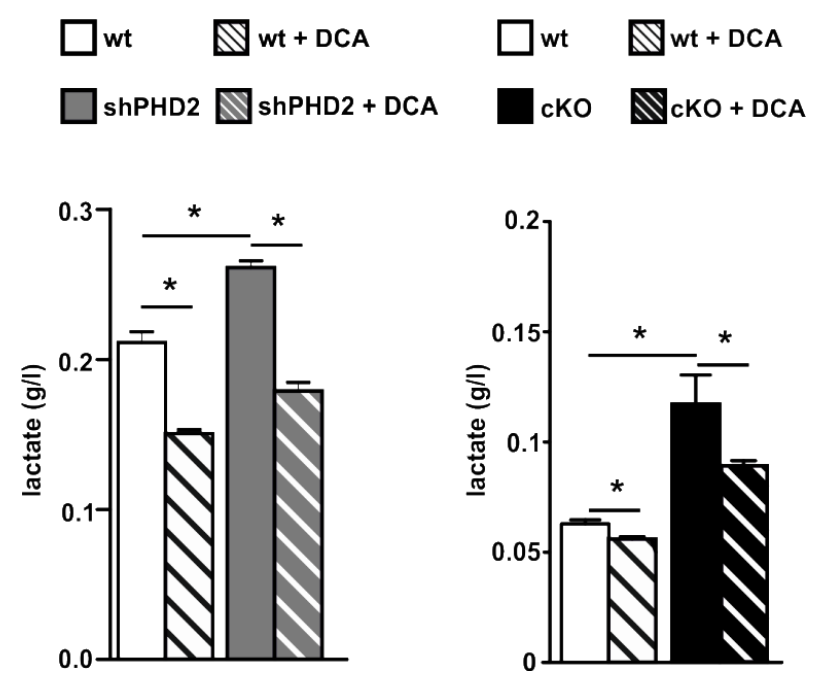

Figure 26: Lactate levels are decreased upon DCA treatment. Lactate levels in the supernatants of RAW wt, shPHD2 RAW, BMDM wt or PHD2 cKO macrophages after incubation of the cells $\pm 5 \mathrm{mM}$ DCA for $24 \mathrm{~h}, \mathrm{n}=4-5$ samples per condition. Graphs show one representative experiment of a set of 3-5 experiments. mean $\pm \mathrm{SEM}, * \mathrm{p}<0.05$

\subsubsection{DCA reduces glycolysis in PHD2 deficient macrophages}

DCA treatment was observed to reverse the effect of PHD2 deficiency on PDH activity and lactate levels. To further confirm that $5 \mathrm{mM}$ DCA treatment for $24 \mathrm{~h}$ is sufficient to reverse the glycolytic phenotype of the PHD2 cKO and shPHD2 cells, also the changes in glycolysis in wt and PHD2 deficient cells treated with DCA were measured. In accordance with the increased PDH activity and the reduced lactate levels, glycolysis observed in PHD2 deficient macrophages was also decreased after DCA treatment when analyzing the ECAR (Fig. 27 A, B). Treatment with DCA reduced the increased acidification rate found in PHD2 cKO and shPHD2 cells. This was observed for both the basal glycolysis and the glycolytic capacity of the cells (Fig. 27 B). DCA treatment also reduced glycolysis and glycolytic capacity in wt cells which is consistent with the observation that also lactate levels are affected by DCA in the wt cells. 
Results
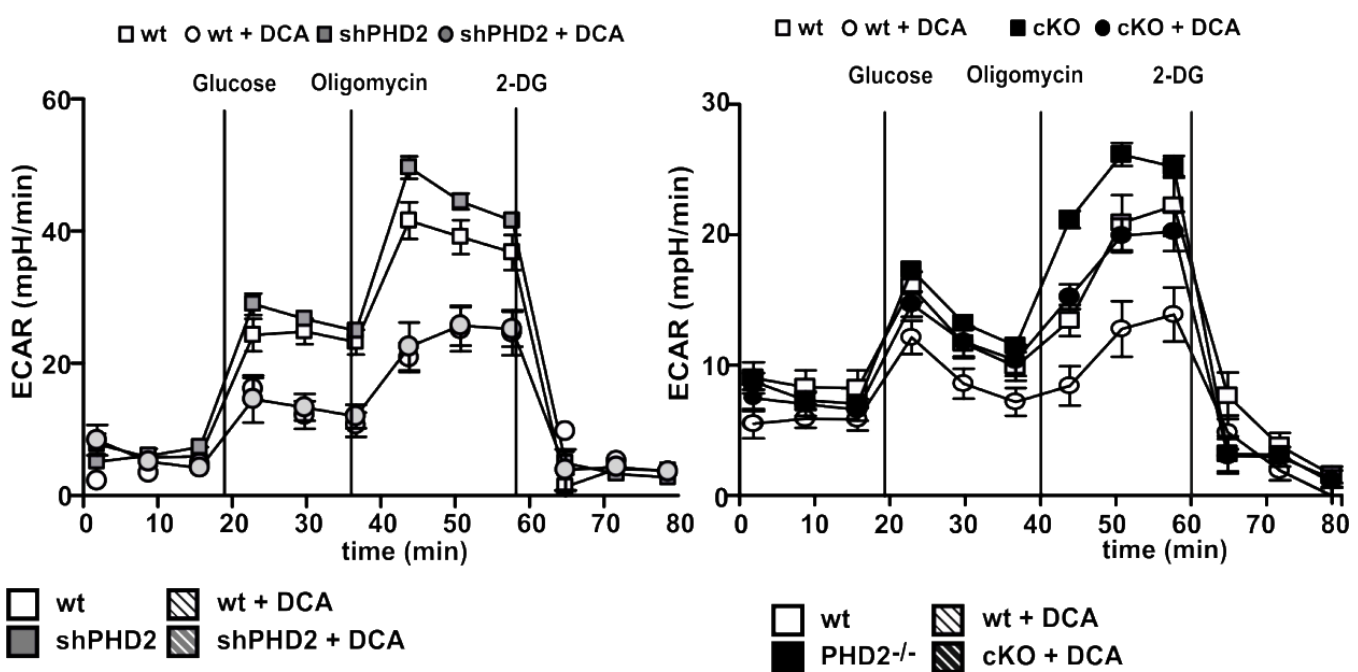

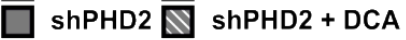

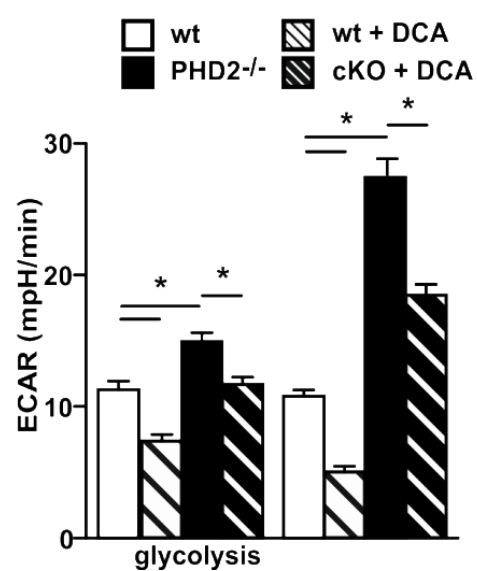

Figure 27: Extracellular acidification rate is reduced by DCA treatment. (A) Extracellular acidification rate (ECAR) was determined in RAW wt, ShPHD2 RAW, BMDM wt and PHD2 cKO cells after addition of glucose, oligomycin and 2-deoxy glucose (2-DG) after an incubation time of $24 \mathrm{~h}$ with $\pm 5 \mathrm{mM}$ DCA. $\mathrm{n}=6-10$ samples per condition. (B) Glycolysis and anaerobic glycolytic capacity were analyzed based on the ECAR after addition of glucose (glycolysis) and oligomycin (glycolytic capacity). The ECAR after 2-DG addition was subtracted from the other rates. Graphs show one representative experiment of a set of 3-4 experiments. mean \pm SEM, * $\mathrm{p}<0.05$ 


\subsection{DCA partially restores macrophage function in PHD2 deficient macrophages.}

\subsubsection{Single cell migration in ShPHD2 cells can be restored by DCA treatment}

As described in the previous chapters, treatment of the metabolic phenotype of PHD2 deficient macrophages could be at least partly reversed with DCA treatment. Treatment with DCA for $24 \mathrm{~h}$ partly re-established wt metabolism and reduced anaerobic glycolysis in these cells. The metabolism of macrophages is closely connected to cellular function. Therefore, it was tested if macrophage function could also be normalized by PDK-inhibition in PHD2 deficient macrophages. For that reason, I analyzed the migratory behaviour of shPHD2 cells that were treated with DCA in a single cell migration assay in response to FCS-containing medium and MDA-MB 231 supernatant over a time course of 6 h (Fig. 28). DCA treatment of shPHD2 RAW cells normalized the migratory behavior in a single cell migration experimental setup and increased it up to wt levels whereas migration in the wt was unaffected by DCA treatment. This effect was even more prominent in the MDA-MB 231stimulated conditions.

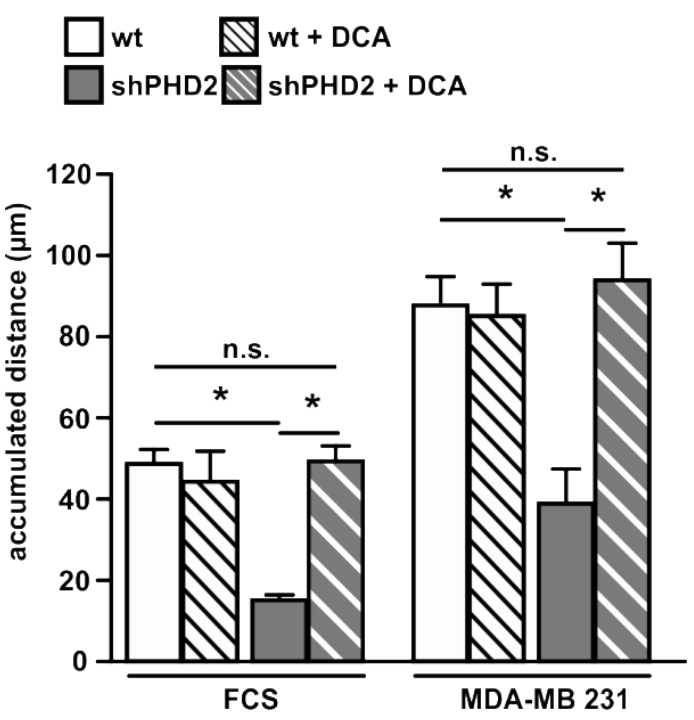

Figure 28: DCA treatment increases migration in a single cell migration experiment. The accumulated migration distance over $6 \mathrm{~h}$ of RAW wt and ShPHD2 RAW cells after incubation of the cells $\pm 5 \mathrm{mM}$ DCA for $24 \mathrm{~h}$ was determined in single cell migration experiments using FCS or MDA-MB 231 conditioned medium as stimulus. $\mathrm{n}=20-68$ cells per condition. Graph shows one representative experiment out of a set of 3 experiments. mean \pm SEM, $* \mathrm{p}<0.05$ 


\subsubsection{Migration in a Boyden chamber assay is not affected by DCA treatment}

In addition to the single cell migration assay I investigated the effect of DCA treatment of PHD2 deficient macrophages in a Boyden chamber assay (Fig.29). The Boyden chamber assay allows to analyze a mixture of cell migration, but also of cell invasion. Therefore, I analyzed if the effect of DCA could be recapitulated in this transwell migration assay. In contrast to the re-established migration observed in response to DCA treatment in the single cell migration experiment, no such effect could be observed in the Boyden chamber assay. Migration of the DCA-treated RAW wt and shPHD2 was not rescued by DCA treatment. macrophages in the Boyden chamber assay. Also DCA treated BMDM wt and PHD2 cKO macrophages did not display an altered transwell migration compared to their untreated controls. This indicates that even though the ability of PHD2 deficient macrophages to migrate in a plane, such as a cell culture dish is enhanced by DCA-treatment, it does not affect the transmigration capacity of the cells.
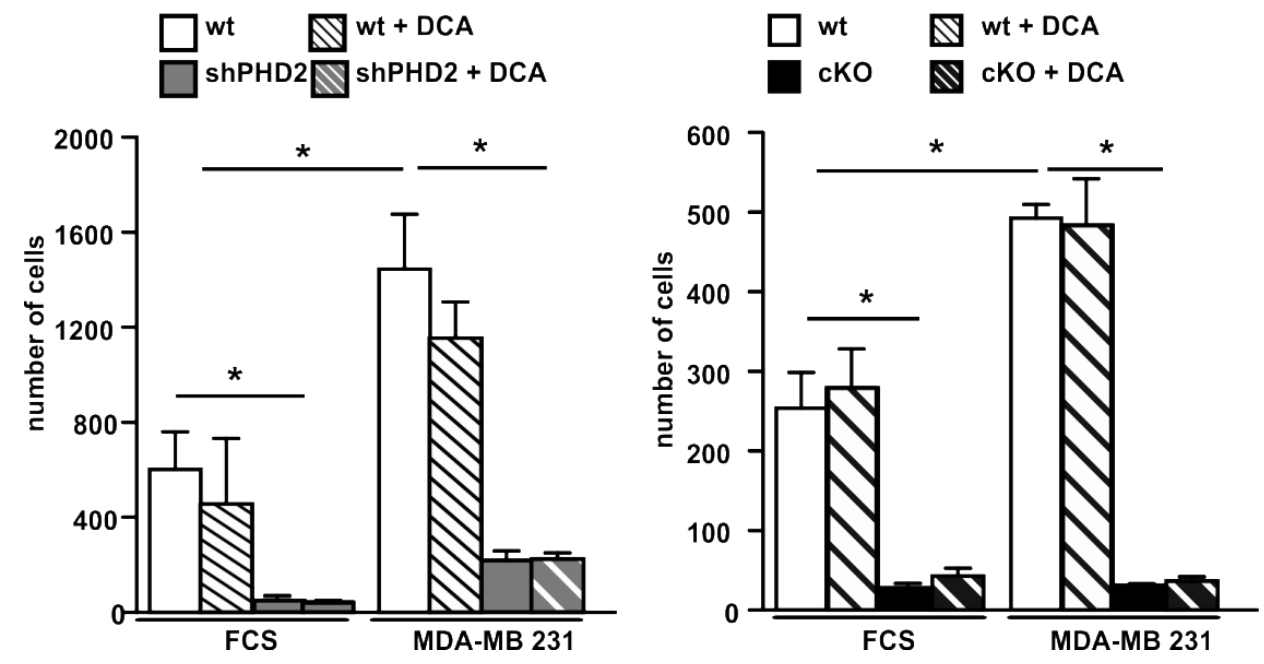

Figure 29: DCA treatment does not affect migration in a Boyden chamber experiment. RAW wt, shPHD2 RAW, BMDM wt and PHD2 cKO cells treated $\pm 5 \mathrm{mM}$ DCA for $24 \mathrm{~h}$ were tested for their migration capacity in Boyden chambers using fetal calf serum (FCS) or conditioned medium of MDA-MB 231 cells as stimulus. $n=6$ (RAW cells), $n=4$ (BMDM). mean \pm SEM, * $\mathrm{p}<0.05$.

\subsubsection{DCA treatment increases phagocytosis in PHD2 deficient macrophages}

Besides migration, phagocytosis is also a suitable indicator of macrophage function. I therefore analyzed if phagocytosis could be influenced by pharmacological inhibition of PDK. Phagocytosis was measured by the uptake of fluorescent beads after $24 \mathrm{~h}$ of DCA treatment. shPHD2 and PHD2 $\mathrm{cKO}$ and the respective wt cells were either treated with $5 \mathrm{mM}$ DCA for 
$20 \mathrm{~h}$ or left untreated, then fluorescent beads were added for additional $4 \mathrm{~h}$ for the cells to phagocytose (Fig. 30). The phagocytosis of the shPHD2 RAW and PHD2 cKO macrophages was increased upon DCA treatment and was almost normalized to wt levels. The capacity of wt RAW and wt BMDMs to perform phagocytosis was not altered by DCA treatment.

Taken together, the data obtained from the effect of DCA treatment in the phagocytosis assay and from the observations made in the single cell migration experiment upon DCA treatment indicate that the metabolic programming via PDK1 is at least partly responsible for the functional impairment observed in PHD2 deficient macrophages.

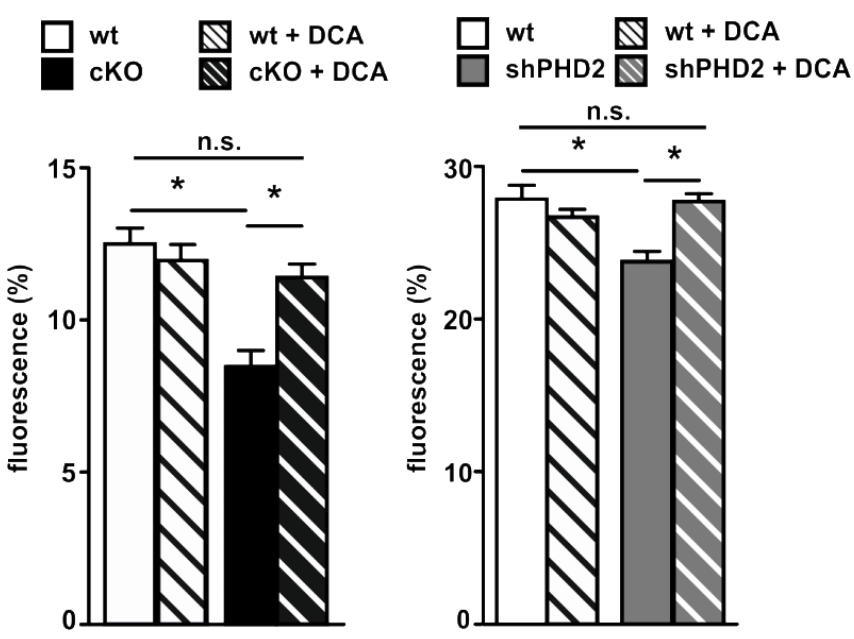

Figure 30: Phagocytosis is reestablished in DCA-treated PHD2 deficient macrophages RAW wt, shPHD2 RAW, BMDM wt and PHD2 cKO cells were incubated $\pm 5 \mathrm{mM}$ DCA for $24 \mathrm{~h}$. Subsequently, the capacity of the cells to phagocytose labeled beads was analyzed. $n=5-8$ samples per condition. Graphs show one representative experiment of a set of $4-6$ experiments. mean $\pm \mathrm{SEM}, * \mathrm{p}<0.05$. 


\section{Discussion}

Macrophages are important effector cells of the innate immune system and play a major role in the phagocytosis of invading pathogens and in the mediation of homeostatic processes (Goren et al., 2009; Mirza et al., 2009). In the past years, it was shown that macrophages have an important role in ischemic disease and that they accumulate inside the tissue after myocardial infarction (Azzawi et al., 2005). Ischemic tissues are characterised by regions of hypoxia and nutrient depletion. This triggers several stimuli leading to tissue cell activation and macrophage invasion into the tissue. Therefore, macrophages have to migrate against the oxygen gradient. To adapt to the scarce oxygen availability, macrophages and other cell types, express a specific gene expression program mediated by HIF. The stabilization of HIF is dependent on the oxygen sensitive regulation of the HIF-1 $\alpha$ subunit by the three PHDs (Bruick and McKnight, 2001; Epstein et al., 2001). PHD2 is the main regulator of HIF-1 $\alpha$ stability in normoxic conditions and deficiency of PHD2 is sufficient to stabilize HIF-1 $\alpha$ and to induce HIF-1 mediated gene expression. Induction of this gene expression program results in the induction of angiogenesis, erythropoiesis, but it also leads to the expression of genes that are involved in metabolism, such as glucose transporters and genes involved in glycolysis (reviewed in (Goda and Kanai, 2012)). Previous studies revealed significant tissue and cell type specific effects of PHD2, but the role of PHD2 in macrophages has not been fully elucidated yet. This study describes an effect of PHD2 deficiency on macrophage metabolism and function.

\subsection{HIF-target genes are differentially expressed in PHD2 cKO and ShPHD2 RAW macrophages}

In PHD2 deficient macrophage models, PHD2 was found to be decreased on RNA and protein level resulting in HIF-1 $\alpha$ stabilization in normoxic conditions. Knockdown of PHD2 or knockout resulted in the induction of HIF target genes in normoxic conditions. The mRNA levels of HIF target gene PHD3 is increased in shPHD2 RAW cells and PHD2 cKO macrophages in normoxic conditions. The compensatory increase of the PHD3 upon a knockdown of PHD2 was already described in other cell- and tissue-specific PHD2 knockout mouse and cell line models (Holscher et al., 2011; Marxsen et al., 2004). In hypoxia, HIF 
strongly induces the expression of PHD3 and to a lesser extent PHD2 (Epstein et al., 2001; Metzen et al., 2005; Pescador et al., 2005). It has been proposed that this is to provide a negative feedback loop to limit the hypoxic response during prolonged hypoxia (del Peso et al., 2003; Minamishima et al., 2009) . HIF-1 $\alpha$ mRNA levels are unaffected by PHD2 deficiency. It has been previously reported that HIF-1 $\alpha$ activity is regulated by protein stabilization rather than regulation of mRNA expression levels (Wenger et al., 1997). HIF-1 $\alpha$ protein levels were even further increased in hypoxia in the shPHD2 RAW cells and PHD2 cKO BMDMs showing that the $80 \%$ reduction in PHD2 levels was not efficient enough to induce maximal HIF-1 $\alpha$ stabilization in normoxia. This is further supported by the fact that HIF target gene expression was further increased in the shPHD2 and PHD2 cKO cells compared to normoxia. RAW wt cells and wt BMDMs induce the expression of HIF target genes in hypoxia which is in line with the literature (Cramer et al., 2003). HIF-1 triggers a differential gene expression which in turn regulates several cellular processes to ensure optimal adaptation of the cell to the limited oxygen availability. This is achieved by genes that affect the metabolism of the cell and switch the source for energy generation from aerobic to anaerobic glycolysis. For example, glucose uptake is increased by upregulation of GLUT1 (Ebert et al., 1995; Hayashi et al., 2004). The glycolytic flux is augmented by the upregulation of PFK1 and PDK1 (Obach et al., 2004; Papandreou et al., 2006). PDK1 inhibits the activity of PDH which catalyzes the conversion of pyruvate to acetyl-CoA (Papandreou et al., 2006). Thus, less acetyl-CoA is provided to the TCA cycle and pyruvate is converted to lactate for energy generation. Another adaptation mechanism is the subunit isoform switch from COX41 to COX4-2 in the mitochondrial complex IV of the respiratory chain (Fukuda et al., 2007). This exchange is mediated by LonP which degrades COX4-1. The switch from COX4-1 to COX4-2 results in an increased efficiency of complex IV regarding the amounts of ATP produced and decreases the mitochondrial oxygen consumption. HIF also triggers BNIP3 expression to induce mitochondrial autophagy and to limit ROS levels (Bruick, 2000; Zhang et al., 2008). All these genes were found to be induced in shPHD2 RAW cells and PHD2 cKO cells. This indicates that the metabolism of these cells is changed. The induction of the genes in PHD2 deficient macrophages was HIF-1-dependent as the genes found to be upregulated in normoxia in the PHD2 cKO and shPHD2 cells were unchanged or decreased in HIF-1 $\alpha /$ PHD2 cKO BMDMs. This indicates that the HIF-1 $\alpha$ stabilization due to PHD2 deficiency is an important factor in the gene expression pattern found in the shPHD2 RAW and PHD2 cKO macrophages. 


\subsection{Macrophage function is altered in PHD2 deficient macrophages}

The main task of macrophages is to recognize and migrate towards foreign bodies, worn-out cells and other debris and to engulf them in order to remove them from the system. Hence, analysis of the migration capacity and the phagocytosis properties of macrophages provide a good indication on the general macrophage fitness.

\subsubsection{Migration and phagocytosis is impaired in PHD2 cKO and shPHD2 macrophages}

The PHD2 deficient shPHD2 RAW and PHD2 cKO macrophages used in this project displayed impaired phagocytosis and migration. The migration of PHD2 deficient macrophages was significantly reduced in a single cell migration experiment and also in a transwell migration assay. The phagocytosis of fluorescently-labeled beads by both RAW cells and BMDMs was decreased in the PHD2 deficient cells. Thus, PHD2 deficiency results in a diminished macrophage function in RAW cells and BMDMs. This impairment in macrophage function was not associated with a reduced proliferation rate in shPHD2 cells indicating that the most basic cellular functions are not affected by PHD2 deficiency. The defect in migration of PHD2 deficient macrophages is in line with other reports (Ikeda et al., 2013; Mamlouk et al., 2014). Mamlouk et al. showed that shPHD2 RAW cell and PHD2 deficient peritoneal macrophage migration toward macrophage chemoattractant protein-1 (MCP-1) as a stimulus was decreased. Additionally, Ikeda et al. reported that in LysM Cre PHD2 ${ }^{\mathrm{fl} / \mathrm{fl}}$ animals, less macrophages infiltrated the aorta in a model of hypertensive cardiovascular remodeling (Ikeda et al., 2013). However, these studies did not provide further insight into the underlying mechanisms. One might argue that the migration and phagocytosis deficit depends on the stabilization of HIF-1 $\alpha$ in PHD2 deficient macrophages. This hypothesis could be tested by analyzing migration and phagocytosis in HIF-1 $\alpha \mathrm{KO}$ macrophages or macrophages that overexpress HIF-1 $\alpha$. Several other groups investigated the role of HIF-1 $\alpha$ in the context of macrophage function. HIF-1 $\alpha$ knockout in myeloid cells was described to decrease migration of peritoneal macrophages in a Boyden chamber (Cramer et al., 2003; Semba et al., 2016). Phagocytosis was also impaired in macrophages lacking HIF$1 \alpha$ (Peyssonnaux et al., 2005). On the other hand, phagocytosis was also impaired in RAW macrophages that overexpress HIF- $1 \alpha$ as shown by the significantly lower intracellular counts of bacteria (Shirato et al., 2009). The opposite findings in PHD2 and HIF-1 $\alpha$-KO models 
stress that the influence of PHD2 and HIF-1 $\alpha$ stabilization on migration and phagocytic capacity is not easily answered and requires more investigation. In this context, it is important to mention that there are indications for PHD dependent alternative substrates (reviewed in (Wong et al., 2013)). PHD dependent alternative substrates might explain why a loss of PHD2 function not always mimics a HIF-1 $\alpha$ gain of function..

\subsubsection{Treatment with DMOG or exposure to hypoxia also reduces migration and phagocytosis of macrophages}

In the RAW wt macrophages, I found single cell migration to be reduced in the single cell migration experiment by exposure to hypoxia or by treatment with the PHD inhibitor DMOG. This resembled the migratory defects found in the shPHD2 RAW cells. The effect of hypoxia on migration is not well established in the literature. Some groups report an increase in migration, other groups find that migration is impaired under hypoxic conditions (Raheja et al., 2011; Semba et al., 2016; Turner et al., 1999; Vogler et al., 2013). Reasons for the divergence in the findings might be differences in the oxygen percentage defined as hypoxia in the studies, the exposure time to hypoxia and the experimental model used. In a macrophage model, it was recently reported that $4 \mathrm{~h}$ of hypoxia $\left(1 \% \mathrm{O}_{2}\right)$ resulted in an increase of thioglycollate-stimulated peritoneal macrophages in a transwell migration assay (Semba et al., 2016). A study conducted with THP1 cells and human peritoneal macrophages found an impairment in migration under hypoxic conditions (Turner et al., 1999). My data indicate that the ability of macrophages to migrate suffers in hypoxia.

Furthermore, the phagocytosis phenotype could be mimicked by exposing the cells to hypoxic conditions and by pharmacological inhibition of the PHDs with DMOG. In contrast to my findings, Anand et al. reported an increased phagocytosis in RAW cells in hypoxic conditions. However, one has to keep in mind the differences in the experimental set up. Whereas I exposed the cells to $24 \mathrm{~h}$ of hypoxia or DMOG, in the report by Anand et. al. the longest exposure time was $8 \mathrm{~h}$. It might well be that the exposure time to hypoxia and thus the time of HIF-stabilization contributes to these opposite findings. It might be that when encountering a region of hypoxia, macrophages first heavily migrate towards the site of hypoxia and start to phagocytose cellular debris, but if the hypoxia is not resolved, the capacity of the macrophages to phagocytose might cease. One possible explanation for this could be that the energy pool of the macrophages might be exhausted by the long term dependence on 
anaerobic glycolysis. This hypothesis is further supported by the fact that long term stabilization of HIF- $\alpha$ by PHD2 deficiency also reduces phagocytosis in my model and that the ATP levels decrease in hypoxia and PHD2 deficient macrophages.

\subsection{PHD2 deficiency in macrophages results in metabolic reprogramming}

Otto Warburg first described that tumor cells in contrast to normal cells, use "fermentation" of glucose to lactate additionally to oxidative phosphorylation and independent from the oxygen availability (Warburg et al., 1927). Thus, tumor cells would use anaerobic glycolysis even in conditions with sufficient oxygen. Anaerobic glycolysis is characterized by a reduction in OCR and an increase in lactate levels. The PHD/HIF-1 pathway was described earlier to mediate a metabolic change that will shift the cellular metabolism form aerobic to anaerobic glycolysis. Therefore, it was analyzed if PHD2 deficiency affects the metabolic profile of the cells.

\subsubsection{Macrophage energy metabolism is altered by PHD2 deficiency}

ShPHD2 RAW cells and PHD2 cKO macrophages had decreased ATP levels compared to the respective wt control cells. Furthermore, ATP levels were even further reduced by hypoxia and by inhibition of PHDs by DMOG. The drop in ATP levels after exposure to hypoxia is in accordance with other studies conducted (Fukuda et al., 2007; Heerlein et al., 2005; Weinberg et al., 2000; Zhang et al., 2008). The ATP levels in the PHD2 deficient cells dropped even further in hypoxic conditions and the hypoxic reduction in ATP was more severe in the PHD2 deficient macrophages compared to wt cells. This shows that the effect of PHD2 deficiency cannot be completely resembled by $24 \mathrm{~h}$ hypoxia and does not show the maximal effect. This might be explained by the fact that the knockdown efficiency of my model is not strong enough to mimic hypoxic conditions completely. Furthermore, a role of the other remaining PHDs in maintaining the ATP pool cannot be excluded. Especially PHD1 has been described to impact cellular metabolism (Aragones et al., 2008).

In hypoxic conditions, cells switch their metabolism in favor of anaerobic glycolysis in order to cope with the scarce oxygen availability. In shPHD2 RAW and PHD2 cKO cells, OCR was found to be decreased and glycolysis was found to be increased. Consequently, lactate levels 
were also elevated. This was also the case when using the PHD inhibitor DMOG. This shows that PHD2 deficient macrophages switch their metabolism to anaerobic glycolysis. An increase in lactate levels in macrophages in hypoxia was also reported by others (Meiser et al., 2015; Semba et al., 2016). Recently it was shown that in the liver PHD2-deficiency induces a glycolytic gene expression profile and increases lactate levels in mouse embryonic fibroblasts (Suhara et al., 2016). This observation is in line with my findings. PHD2 deficient RAW cells and BMDMs display all features which indicate that the cells underwent metabolic reprogramming. This means that PHD2 deficient macrophages display a decreased oxygen consumption rate with increased glycolysis which is accompanied by reduced ATP levels. Taken together, my data indicates that PHD2-deficiency in macrophages is sufficient to trigger a glycolytic phenotype in normoxic conditions which is comparable to the Warburg effect.

Changes in metabolism of macrophages were described to be associated with macrophage polarization. M1 polarized macrophages were shown to be more glycolytic and to induce an attenuation of the mitochondrial respiratory chain (reviewed in (Galvan-Pena and O'Neill, 2014)). The accumulation of TCA cycle intermediates results in an increase in cellular ROS levels which is used for bacterial killing. Takeda et. al. showed in a heterozygous PHD $2^{+/-}$mouse model that PHD2-deficiency in macrophages triggers M2 polarization (Takeda et al., 2011). M2 polarized macrophages are described to use oxidative phosphorylation as the major ATP source (reviewed in (Galvan-Pena and O'Neill, 2014)). This is in contrast to my findings in the PHD2 cKO and shPHD2 RAW macrophages given that the glycolytic phenotype in my data would rather indicate a M1 polarization of PHD2 deficient macrophages. Data generated by Dr. Katja Farhat (unpublished, laboratory of Prof. Katschinki) revealed that macrophage polarization is unaltered in PHD2 cKO macrophages. This shows that the metabolic switch observed in PHD2 deficient macrophages is independent from a switch in polarization.

It was reported previously that in response to hypoxia, HIF triggers the induction of BNIP3 which in turn induces mitochondrial autophagy to prevent increased ROS levels (Bellot et al., 2009; Zhang et al., 2008). In PHD2 deficient macrophages, I found an induction of BNIP3 mRNA, but the mitochondrial number of these macrophages remained unchanged indicating that mitochondrial autophagy is not increased in these cells. Mitochondrial function has an important role in migration as inhibition of the mitochondrial complex I by rotenone reduced migration of RAW wt macrophages severely in a single cell migration experiment. 
Mitochondria are the major source of cellular ATP generation, thus it is not surprising that an energy consuming process such as cell migration, is affected by decreased mitochondrial function. In line with the present study, a role of mitochondria was reported earlier for the migratory capacity of MDA-MB 231 cells (Zhao et al., 2013). When treated with mitochondrial inhibitors CCCP and oligomycin, cellular migration was reduced. Thus, interfering with the mitochondrial respiratory chain inhibits the migratory capacity of the cells. In the shPHD2 and PHD2 cKO macrophages, mitochondrial respiration is downregulated. It might be that the reduced oxidative phosphorylation in these cells contributes to the impaired migration.

\subsubsection{ROS levels are differently altered in ShPHD2 RAW isolated mitochondria and total cells.}

The amount of ROS production in hypoxia is still under debate whereas some studies report a decrease in mitochondrial ROS in response to hypoxia (Archer et al., 1989; Hoffman et al., 2007; Paky et al., 1993), other groups report an increase in ROS levels (Bell et al., 2007; Chandel et al., 2000; Guzy et al., 2005). The increase of ROS is proposed to result from a hypoxia induced conformational change in complex III (ubiquinol cytochrome c oxidoreductase) of the respiratory chain (Guzy et al., 2005; Mansfield et al., 2005). This change is supposed to enhance the interaction between the ubisemiquinone radical and $\mathrm{O}_{2}$ thereby increasing superoxide formation. One hypothesis for the increase of mitochondrial ROS production in hypoxic conditions is that the increased abundance of ROS results in the accumulation of iron in the $\mathrm{Fe}^{3+}$ - state (reviewed in (Taylor, 2008)). $\mathrm{Fe}^{3+}$ cannot be used as a cofactor by the PHDs, thereby attenuates PHD activity and thus hypoxic ROS would increase HIF- $\alpha$ stabilization. Indeed, adding ROS such as hydrogen peroxide was sufficient to trigger HIF- $\alpha$ stabilization in normoxic conditions (Bell et al., 2007). One hypothesis for the decrease of ROS in hypoxic conditions proposed that the inhibition of mitochondria in hypoxia results in a reduction in oxygen consumption which results into less oxygen used by the mitochondria (reviewed in (Taylor, 2008)). This reduction in mitochondrial oxygen utilization will increase the availability of oxygen in the cytosol and can be used by the PHDs to limit the hypoxic response. In my PHD2 deficient models, I found mitochondrial ROS production to be reduced. The role of PHD2 in mitochondrial ROS production has not been described earlier. Mitochondrial ROS production, at least in isolated mitochondria was not increased in 
the model used here. Regarding the hypothesis that mitochondria reduce ROS in order to repress HIF signaling, this would mean that long-term HIF- $\alpha$ stabilization, as induced by PHD2 deficiency, triggers a mechanism that reduces mitochondrial ROS. This might happen in order to reduce HIF signaling by PHDs. The reduction in mitochondrial ROS production seems to be independent from the actual oxygen availability within the cell as the experiment was performed at normoxic conditions. It was shown by others that isolated rat mitochondria reduce their ROS production with decreasing oxygen concentration (Hoffman et al., 2007). Assuming that the mitochondrial ROS production is dependent on HIF-1 $\alpha$ stabilization, this is line with the finding that mitochondria isolated from PHD2 deficient macrophages display reduced ROS levels.

Interestingly, in contrast to the reduced mitochondrial ROS production, I found an increase in total cellular ROS levels. This is surprising given that the mitochondria are widely assumed to be a major source of cellular ROS. In quantitative manners, it was described at least for the liver that the endoplasmatic reticulum and peroxisomes have a greater capacity to produce the ROS $\mathrm{H}_{2} \mathrm{O}_{2}$ (Boveris et al., 1972). For other cell types, it is evident that the mitochondria are an important ROS source, but the actual contribution of mitochondrial ROS to the net cellular ROS is missing (reviewed in (Brown and Borutaite, 2012)). The ROS elevation observed in the total cells might also be due to the increased activity of ROS-producing enzymes, such as nitric oxidases, lipoxygenases and NADPH oxidases (Nox) (reviewed in (Holmstrom and Finkel, 2014)). Especially, the Nox enzymes were described to have a function in hypoxic conditions. Nox4 levels were found to be increased in cardiomyocytes and in pulmonary vasculature in hypoxic conditions (Mittal et al., 2007; Wang et al., 2014). This upregulation had a significant role in the promotion of microvascular angiogenesis after hypoxia/reperfusion injury. Also Nox 2 mediated ROS production was shown to have a role in the ischemic response in the mobilization and expansion of progenitor cells in the bone marrow (Urao et al., 2012). These mechanisms might also contribute to an increased ROS production. 


\subsection{The metabolic alterations observed in PHD2 deficient macrophages depend at least partially on PDK1}

PDK1 is a key regulator in glucose metabolism mediating the switch from aerobic to anaerobic respiration. This ensures metabolic homeostasis and saves oxygen in hypoxic conditions. PDK $1^{-/-}$mice with a cardiomyocyte specific knockout of PDK1 die of heart failure between 5 and 11 weeks of age (Mora et al., 2003). Cardiomyocytes isolated from these mice were notably more sensitive to hypoxia compared to wt cardiomyocytes stressing the importance of PDK1 in the adaptation to hypoxia.

PDK1 levels and the amount of phosphorylated PDH are increased in shPHD2 RAW cells and PHD2 cKO BMDMs on protein levels. This correlated with a decreased PDH activity in shPHD2 RAW cells in normoxic conditions. The phenotype of the shPHD2 cells could be mimicked by treating the wt cells with DMOG or by exposing the cells to hypoxia. The reduction of PDH flux in resting macrophages in hypoxic conditions had been reported earlier and is in line with my findings (Meiser et al., 2015). The PDH activity in the shPHD2 cells is still reduced compared to wt cells in the hypoxic conditions indicating that PDH activity is not completely blocked in the wt cells in hypoxia. This is in line with the finding that lactate levels are still increased and ATP levels are reduced in the shPHD2 cells compared to wt after $24 \mathrm{~h}$ of hypoxia. One possible explanation for this phenotype might be that the effect of hypoxia is more severe in cells that already have an increased HIF-1 $\alpha$ stabilization due to PHD2 deficiency. In a wt situation, HIF-1 induces PHD2 and PHD3 during hypoxia thereby limiting the hypoxic response. This pathway will be hampered in the PHD2 deficient cells resulting in an augmented hypoxic response. This is furthermore supported by the fact that the induction of HIF-target genes in hypoxic conditions was generally increased in the shPHD2 and PHD2 cKO macrophages compared to wt cells.

To obtain further insight into the role of PDK1 in the metabolic shift observed in PHD2 deficient macrophages, I treated the cells with the PDK-inhibitor dichloroacetate (DCA). To date, there are four known PDK isoforms in mammals, i.e. PDK1-4. PDKs inactivate the PDH complex by phosphorylating it at three serine residues (Ser232, Ser293 and Ser300) (Rardin et al., 2009). PDK1 is the only one of the PDKs which phosphorylates all three sites (Kolobova et al., 2001). Mutagenesis studies on recombinant human PDH revealed that phosphorylation on any of the three serine residues is sufficient to inhibit the dehydrogenase activity in vitro (Korotchkina and Patel, 1995). PDKs are activated by high abundances of acetyl Co-A, 
NADH and ATP and are inhibited by high amounts of ADP and pyruvate (Kerbey et al., 1976). The pyruvate mimetic DCA is just $150 \mathrm{Da}$ in size and therefore easily crosses the cellular membrane (Michelakis et al., 2008). Binding of DCA to PDK1 triggers conformational changes in the nucleotide- and lipoyl-binding pockets of PDK1 (Kato et al., 2007). This results in inactivation of the kinase activity. DCA is the most commonly used inhibitor of PDK enzymes. In the literature, it is used in a wide range from $0.5 \mathrm{mM}$ to 100 $\mathrm{mM}$ (reviewed in (Kim et al., 2006)), mostly to induce a cytotoxic effect on cancer cells which depend heavily on energy generation by lactate production. Bonnet et. al. showed that DCA induces apoptosis in cancer cells, but not in normal cells stressing the dependence of cancer cells on glycolysis (Bonnet et al., 2007). Inactivation of PDKs results in a shift of cellular metabolism by forcing more energy to be generated by feeding acetyl-CoA into the respiratory chain. DCA is also described to have implications in inhibiting mitochondrial hyperpolarization by reactivations of a mitochondrial potassium-ion channel axis and inhibit tumor growth (Bonnet et al., 2007).

In the RAW cell line model, DCA had no significant effects on the PDH activity of wt macrophages. In the shPHD2 cells on the other hand, it significantly increased PDH activity. Thus, the repression of PDH activity observed in the shPHD2 RAW cells can be reestablished by inhibiting PDK by DCA. The increased activity of PDH should result in an increase in oxidative phosphorylation and a decrease in lactate levels, thereby reverting the metabolic shift of the PHD2 deficient macrophages

\subsection{Inhibition of PDK1 by DCA partially reestablishes wt phenotype in PHD2 deficient macrophages}

PHD2 deficient shPHD2 RAW cells and PHD2 cKO cells displayed a reduction in ATP levels, a depressed oxygen consumption rate and an increase in glycolysis. This metabolic phenotype was at least partly reversible by inhibition of PDK1. Inhibiting PDK1 with DCA was sufficient to decrease lactate levels and glycolysis and to restore the migratory capacity in a single cell migration experiment and phagocytosis. This indicates that PDK1 is the important factor mediating the metabolic changes and functional alterations in PHD2 deficient macrophages. Previous studies revealed an important role of PDK1 in macrophages (Tan et al., 2015). Even though, no effect of PDK1 induction on macrophage polarization could be observed in this model, the described regulation of glucose metabolism is in line with my 
findings. In the model used in the presented thesis, inhibition of PDK1 by DCA resulted in increased PDH activity in shPHD2 RAW cells that partly resembled the PDH activity of wt cells. Furthermore, DCA treatment also resulted in a reduction in glycolysis and in lactate levels. Functionally, it increased the phagocytosis of shPHD2 and PHD2 cKO macrophages. In addition to that, also single cell migration was reestablished in the shPHD2 cells. Interestingly, inhibition of PDK1 by DCA was not sufficient to reestablish migration in PHD2 deficient macrophages in a Boyden chamber experimental set up. This indicates that the mechanisms involved in transwell migration are not affected by the metabolic switch, but rather via different mechanisms. One possible explanation could be that PHD2 deficient macrophages display an alternate set of chemokine receptors that is expressed independently from the metabolic changes. Chemokines are a family of small proteins that serve as stimulus for leukocyte migration. These chemokines bind to the chemokine receptors and thereby trigger cell migration. Hypoxia was described earlier to influence the expression of chemokine receptors (Bosco et al., 2006; Schioppa et al., 2003). For example, it was reported in human monocytes that the expression of CXC chemokine receptor (CXCR) 4 is upregulated in hypoxic conditions (Schioppa et al., 2003). Furthermore, a transcriptome screen of primary human macrophages revealed that the chemokine receptors CCR5, CCR1 and CCR2 are downregulated in hypoxia (Bosco et al., 2006). The shPHD2 cells have an increased expression of HIF-1 $\alpha$ in normoxia. Thus, chemokine receptors might be differently regulated in the shPHD2 cells. The expression of chemokine receptors would not be affected by the inhibition of PDK1 and hence, no effect on transmigration by DCA treatment could be observed.

Transmigration of macrophages is a very delicate process that involves cytoskeletal rearrangements and the activation of several pathways resulting in the polarization of the macrophage (reviewed in (Imhof and Aurrand-Lions, 2004)). Effective migration requires the formation of lamellipodia in the anterior region of the cell as well as the formation of uropod at the end of the macrophage. Thus, it might be possible that PHD2 deficiency affects macrophage transmigration capacity by a different arrangement of the cytoskeleton. The cytoskeletal rearrangements involve the rearrangements of actin microfilaments (F-actin) which control cell mobility. Actin polarization is regulated by numerous proteins, the best studied are the small GTPases of the RhoA family, their regulators and their effector proteins (reviewed in (Ridley, 2015)). The small GTPases cycle between a GDP bound (inactive) and a GTP-bound (active) state. The best characterized small GTPases are RhoA, Rac1 and 
Cdc42. They are needed for the organization of the actin cytoskeleton (RhoA), lamellipodia (Rac1) and filopodia (Cdc42). Cytoskeletal rearrangements in hypoxia mediated by i.e. the RhoA GTPase pathway have been described for several cell types and might provide a possible explanation for the observed phenotype (Choi et al., 2015; Raheja et al., 2011; Vogler et al., 2013). Inhibition of PDK1 by DCA results in partially reestablished wt metabolism of the PHD2 deficient macrophage, but as these metabolic changes do not affect the cytoskeleton of the cells, it might explain why macrophage transmigration is not rescued by metabolism reestablishment.

\subsection{PHD2 might provide an useful tool to influence macrophage metabolism and function}

The PHD2 deficient macrophage models used in this project exhibited impairment in migration and phagocytosis. This indicates that PHD2 has a role in the mediation of macrophage function. In inflammation, the response of these macrophages is likely to be altered compared to wt macrophages. It remains to be investigated if PHD2 positively or negatively regulates macrophage function in inflammation. PHD2 deficient macrophages might migrate slower to the site of inflammation and once there, these macrophages will contribute less to the clearance of the affected tissue as they are less capable to phagocytose. Macrophages are crucial for the tissue remodeling after myocardial infarction, but they can also be detrimental to the healing process (reviewed in (Liu et al., 2016)). Macrophages accumulate in the infarcted area and are involved in phagocytosis, angiogenesis, inflammation and collagen deposition. Several groups reported an effect of PHD2 deficiency in macrophages in different disease models (Ikeda et al., 2013; Mamlouk et al., 2014; Takeda et al., 2011). For example in a cancer model, it was shown that myeloid specific PHD2 knockout mice exhibited a reduced tumor volume (Mamlouk et al., 2014). This finding was explained by the overall downregulation of cytokines which included pro-inflammatory as well as antiinflammatory cytokines. In a model of pharmacologically induced hypertension, PHD2 deficiency in macrophages attenuated infiltration of macrophages and induced less inflammation and fibrosis which preserved heart function in a myeloid specific PHD2 KO (Ikeda et al., 2013). Additionally, it was also described that PHD2 $2^{+/-}$mice were protected from myocardial ischemia and exhibited an increased viability of ischemic tissue in a model of hindlimb ischemia. This was reported to be associated with a M2 polarization of the PHD2 
deficient macrophages (Takeda et al., 2011). These reports would argue for a beneficial effect of PHD2 deficiency in macrophages. On the other hand, macrophages were also described to be essential to proper tissue repair after myocardial infarction (MI) (Nahrendorf et al., 2011). The removal of dead cells and debris by macrophage phagocytosis is important for the resolution of the inflammation. Therefore, less macrophages at the site of inflammation which at the same time have a reduced rate of phagocytosis might also result in a worse outcome. The defect in macrophage function described in this project initiated another project investigating the effect of PHD2 $\mathrm{cKO}$ macrophages in a model of MI. Unpublished data generated by Angelika Beneke (laboratory of Prof. Katschinski) demonstrate that in the PHD2 cKO mice, the migration kinetics of the PHD2 deficient macrophages into the infarcted heart is shifted. This might indicate a delayed migration of the PHD2 deficient macrophages into the tissue and would be in line with my data. Furthermore, Angelika Beneke also found a significantly decreased heart function at day 7 after myocardial infarction which indicates that inhibition of PHD2 in macrophages might be disadvantageous in the context of MI. Thus, the effect of PHD2 deficiency on macrophages in disease is not easily answered and requires more investigation.

PHD2 deficiency was sufficient to induce an anaerobic glycolytic metabolism in macrophages in normoxic conditions. The metabolism of macrophages was recently described to be connected to the effector function of the macrophages (Mills and O'Neill, 2016). In the case of PHD2 deficiency, the glycolytic phenotype is associated with a decreased abundance of ATP and with a defect in macrophage function. Thus, it might be possible that the function of macrophages can be regulated by metabolic changes due to PHD2 deficiency.

The impairment in migration and phagocytosis could be mimicked by exposing the cells to hypoxia or by treating them with the PHD inhibitor DMOG. DMOG is a competitive $\alpha$ ketoglutarate analogue. Inhibiting the PHD/HIF pathway using $\alpha$-ketoglutarate mimetics is a promising strategy for the development of new drugs. In fact, several PHD inhibitors (PHI) entered clinical trials for the treatment of different diseases. Roxadustat/FG-4592 recently entered stage III clinical trials for chronic kidney disease patients in order to treat renal anemia, but also other PHI are currently tested (reviewed in (Macdougall, 2012; Maxwell and Eckardt, 2015)). Until now, one of the used PHI, the PHI FG-226 was stopped to be tested because one of the patients developed severe hepatitis (reviewed in (Maxwell and Eckardt, 2015)). The results of the phase III trials should be obtained within the next three years and 
will give more information about possible applicability and adverse effects of PHIs. In addition to clinical trials, there is also plenty of preclinical evidence that the induction of the HIF pathway by PHI may be beneficial in tissue protection, for example during myocardial ischemia (reviewed in (Bishop and Ratcliffe, 2015) and (Vogler et al., 2015)).

The metabolic reprogramming induced by PHD2 was reversible by inhibition of PDK1. This shows that the changes in metabolism are indeed crucial for the observed impairment in macrophage function. PDK1 was earlier described to be involved in macrophage function (Kawano et al., 2012). Myeloid specific PDK1 ${ }^{-/-}$KO mice on a high fat diet showed an increased accumulation of M1 polarized macrophages inside fat tissue. Furthermore, PDK1 was found to be involved in the migration of macrophages. The observations by Kawano et. al. further stress the importance of PDK1 in macrophage migration as PDK1 deficient macrophages migrated significantly more compared to controls. In my model, PHD2 deficiency is associated with increased PDK1 levels and decreased migration. The correlation between the level of PDK1 and migratory capacity is in line with my findings, even though transwell migration was not affected by PDK1 inhibition in my model.

The anaerobic glycolytic phenotype was found to be reversible by DCA treatment. DCA treatment was also found to reestablish phagocytosis and single cell migration in the PHD2 deficient macrophages which would implicate that DCA might be a useful tool in modulating macrophage function. The most extensively studied PDK inhibitor is DCA and it is used to reduce congenital lactic acidosis in children with mitochondrial disorders (Stacpoole, 1989; Stacpoole et al., 2006). Additionally, DCA is discussed as a treatment option for cancer therapy (reviewed in (Michelakis et al., 2008)). The Warburg effect which is characterised by the utilization of anaerobic glycolysis in the presence of oxygen depends on the shunting of acetyl-CoA away from the mitochondria. This process is mediated by PDK1. Inhibiting PDK1 in cancer cells therefore reverses the Warburg effect and results in reduced proliferation and increased apoptosis of tumor cells. However, DCA was also shown to induce neurotoxicity in adults in a small blinded placebo controlled study that was conducted in patients with MELAS syndrome (Kaufmann et al., 2006). MELAS syndrome is defined by mitochondrial myopathy, encephalopathy, lactic acidosis and stroke-like episodes. In this study, it was observed that almost all the patients treated with DCA developed peripheral neuropathy. Thus inhibition of PDK1 using DCA would reestablish macrophage function, but due to its toxicity 
the use is limited. PHD inhibitors, however, are currently tested in late stage clinical trials and until now seem to be relatively safe (reviewed in (Maxwell and Eckardt, 2015).

Taken together, my data indicates that cellular metabolism and cell function can also be influenced by PHD2 in macrophages. PHD inhibitors have reached late stage clinical trials and seem to be relatively safe. The most commonly used PDK inhibitor DCA on the other hand was found to have severe adverse side effects in adults. Therefore targeting PHD2 might provide a better way to influence cellular metabolism and macrophage function than does targeting PDK directly. 


\section{Conclusion}

In the presented thesis the effect of PHD2 on metabolism and function of macrophages was described (Fig. 31). For this, primary BMDMs which harbor a homozygous knockout of PHD2 and a RAW cell line that stably express shRNA against PHD2 were used. Both models showed a decrease in PHD2 expression of approximately $80 \%$. PHD2 deficient macrophages showed a stabilization of HIF-1 $\alpha$ and increased HIF target gene expression. The induction of the HIF target gene PDK1 led to a metabolic reprogramming which was associated with increased lactate levels and glycolysis, depressed OCR and a decrease in migration and phagocytic capacity. The decrease in oxygen consumption was not due to a reduced number of mitochondria. Furthermore, I found mitochondrial ROS production to be reduced and total cellular ROS to be increased. The underlying mechanisms to these seemingly contradictory findings remain to be investigated. However, my data clearly show that PHD2 deficiency negatively regulated PDH activity.

A reduction in migration and phagocytosis, a decrease in ATP levels and an increase in lactate levels was more prominent in hypoxic conditions than in PHD2 deficient cells in normoxia. This suggests that the PHD2 knockdown efficiency was either not strong enough to induce the maximal effect or that additional mechanisms contribute to metabolic adaptation in hypoxia. The changes in metabolism in PHD2 deficient macrophages were dependent on PDK1 activity. Inhibiting PDK1 with DCA was sufficient to decrease lactate levels and glycolysis and to restore the migratory capacity in single cell migration experiments and to restore phagocytosis. Transwell migration is supposedly mediated via a different mechanism because it was not affected by PDK inhibition. The underlying mechanism of this difference could not be answered within the work scope of this project and remains to be elucidated.

In conclusion, my data indicate that PHD2 plays an important role in macrophage metabolism and function. PHD2 influences the balance between aerobic and anaerobic glycolysis via the expression of PDK1. Therefore, targeting PHD2 might provide a useful approach to influence the immune function of macrophages via metabolic reprogramming. 


\section{Conclusion}
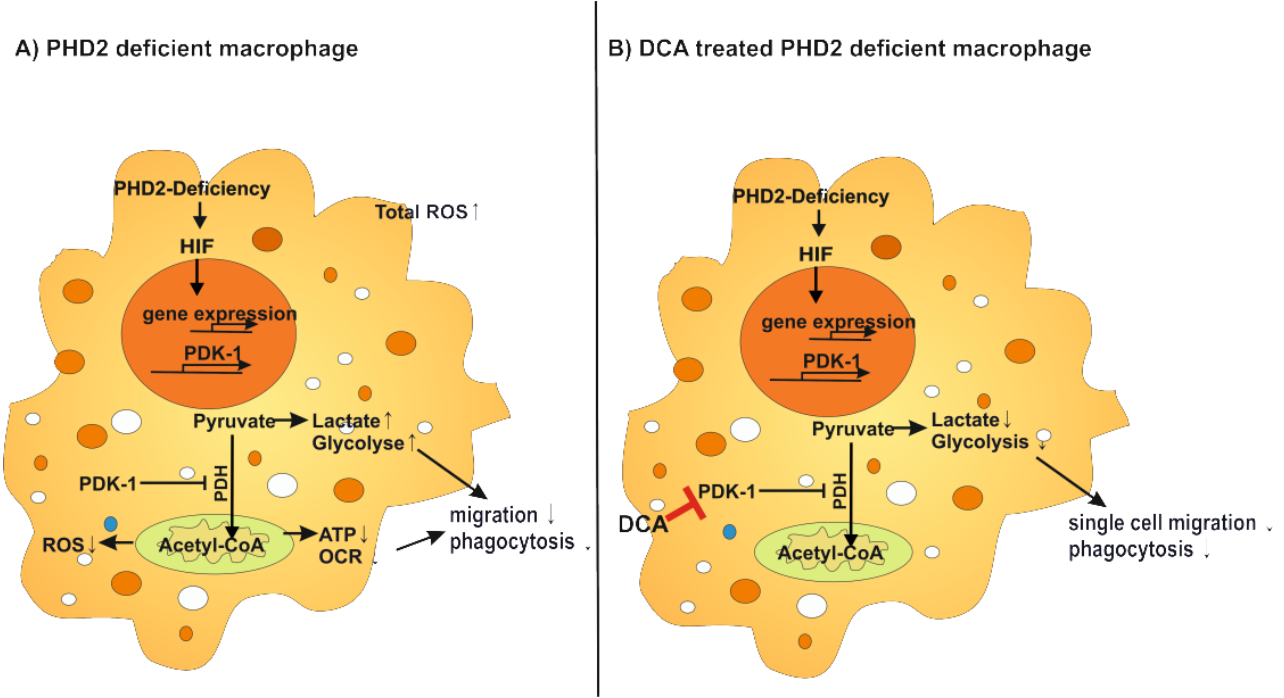

Figure 31: PHD2 deficient macrophage undergo metabolic reprogramming dependent on PDK1. (A) PHD2 deficiency results in HIF-1 $\alpha$ stabilization and HIF target gene expression, such as PDK1. PDK1 inhibits PDH which leads to a reduction of mitochondrial respiration which depends on acetyl-CoA as a substrate. Overall, this results in a reduction of cellular ATP and mitochondrial oxygen consumption. The mitochondrial ROS is reduced whereas total cellular ROS levels are increased. The inhibition of PDH by PDK1 results in an increased quantity of pyruvate utilized for energy generation by anaerobic glycolysis and thus lactate levels increase. Furthermore, PHD2 deficient macrophages have reduced migration and impaired phagocytosis. (B) Inhibiting PDK1 by DCA treatment reinstalled PDH activity and reduced glycolysis and thus normalized lactate levels. Furthermore, DCA was found to at least partly reestablish migration and phagocytosis in PHD2 deficient macrophages. 


\section{References}

Adluri, R.S., M. Thirunavukkarasu, N.R. Dunna, L. Zhan, B. Oriowo, K. Takeda, J.A. Sanchez, H. Otani, G. Maulik, G.H. Fong, and N. Maulik. 2011. Disruption of hypoxia-inducible transcription factor-prolyl hydroxylase domain-1 (PHD-1-/-) attenuates ex vivo myocardial ischemia/reperfusion injury through hypoxia-inducible factor-1alpha transcription factor and its target genes in mice. Antioxid Redox Signal. 15:1789-97.

Appelhoff, R.J., Y.M. Tian, R.R. Raval, H. Turley, A.L. Harris, C.W. Pugh, P.J. Ratcliffe, and J.M. Gleadle. 2004. Differential function of the prolyl hydroxylases PHD1, PHD2, and PHD3 in the regulation of hypoxia-inducible factor. J Biol Chem. 279:38458-65.

Aragones, J., M. Schneider, K. Van Geyte, P. Fraisl, T. Dresselaers, M. Mazzone, R. Dirkx, S. Zacchigna, H. Lemieux, N.H. Jeoung, D. Lambrechts, T. Bishop, P. Lafuste, A. Diez-Juan, S.K. Harten, P. Van Noten, K. De Bock, C. Willam, M. Tjwa, A. Grosfeld, R. Navet, L. Moons, T. Vandendriessche, C. Deroose, B. Wijeyekoon, J. Nuyts, B. Jordan, R. Silasi-Mansat, F. Lupu, M. Dewerchin, C. Pugh, P. Salmon, L. Mortelmans, B. Gallez, F. Gorus, J. Buyse, F. Sluse, R.A. Harris, E. Gnaiger, P. Hespel, P. Van Hecke, F. Schuit, P. Van Veldhoven, P. Ratcliffe, M. Baes, P. Maxwell, and P. Carmeliet. 2008. Deficiency or inhibition of oxygen sensor Phd1 induces hypoxia tolerance by reprogramming basal metabolism. Nat Genet. 40:17080.

Arany, Z., L.E. Huang, R. Eckner, S. Bhattacharya, C. Jiang, M.A. Goldberg, H.F. Bunn, and D.M. Livingston. 1996. An essential role for p300/CBP in the cellular response to hypoxia. Proc Natl Acad Sci U S A. 93:12969-73.

Archer, S.L., D.P. Nelson, and E.K. Weir. 1989. Simultaneous measurement of O2 radicals and pulmonary vascular reactivity in rat lung. J Appl Physiol (1985). 67:1903-11.

Azzawi, M., S.W. Kan, V. Hillier, N. Yonan, I.V. Hutchinson, and P.S. Hasleton. 2005. The distribution of cardiac macrophages in myocardial ischaemia and cardiomyopathy. Histopathology. 46:314-9.

Bell, E.L., T.A. Klimova, J. Eisenbart, C.T. Moraes, M.P. Murphy, G.R. Budinger, and N.S. Chandel. 2007. The Qo site of the mitochondrial complex III is required for the transduction of hypoxic signaling via reactive oxygen species production. J Cell Biol. 177:1029-36.

Bellot, G., R. Garcia-Medina, P. Gounon, J. Chiche, D. Roux, J. Pouyssegur, and N.M. Mazure. 2009. Hypoxia-induced autophagy is mediated through hypoxia-inducible factor induction of BNIP3 and BNIP3L via their BH3 domains. Mol Cell Biol. 29:2570-81.

Berra, E., E. Benizri, A. Ginouves, V. Volmat, D. Roux, and J. Pouyssegur. 2003. HIF prolylhydroxylase 2 is the key oxygen sensor setting low steady-state levels of HIF-1alpha in normoxia. EMBO J. 22:4082-90.

Berra, E., A. Ginouves, and J. Pouyssegur. 2006. The hypoxia-inducible-factor hydroxylases bring fresh air into hypoxia signalling. EMBO Rep. 7:41-5.

Bishop, T., D. Gallagher, A. Pascual, C.A. Lygate, J.P. de Bono, L.G. Nicholls, P. OrtegaSaenz, H. Oster, B. Wijeyekoon, A.I. Sutherland, A. Grosfeld, J. Aragones, M. Schneider, K. van Geyte, D. Teixeira, A. Diez-Juan, J. Lopez-Barneo, K.M. Channon, P.H. Maxwell, C.W. Pugh, A.M. Davies, P. Carmeliet, and P.J. Ratcliffe. 2008. 
Abnormal sympathoadrenal development and systemic hypotension in PHD3-/- mice. Mol Cell Biol. 28:3386-400.

Bishop, T., and P.J. Ratcliffe. 2015. HIF hydroxylase pathways in cardiovascular physiology and medicine. Circ Res. 117:65-79.

Biswas, S.K., and A. Mantovani. 2012. Orchestration of metabolism by macrophages. Cell Metab. 15:432-7.

Bonnet, S., S.L. Archer, J. Allalunis-Turner, A. Haromy, C. Beaulieu, R. Thompson, C.T. Lee, G.D. Lopaschuk, L. Puttagunta, G. Harry, K. Hashimoto, C.J. Porter, M.A. Andrade, B. Thebaud, and E.D. Michelakis. 2007. A mitochondria-K+ channel axis is suppressed in cancer and its normalization promotes apoptosis and inhibits cancer growth. Cancer Cell. 11:37-51.

Bosco, M.C., M. Puppo, C. Santangelo, L. Anfosso, U. Pfeffer, P. Fardin, F. Battaglia, and L. Varesio. 2006. Hypoxia modifies the transcriptome of primary human monocytes: modulation of novel immune-related genes and identification of CC-chemokine ligand 20 as a new hypoxia-inducible gene. J Immunol. 177:1941-55.

Boveris, A., N. Oshino, and B. Chance. 1972. The cellular production of hydrogen peroxide. Biochem J. 128:617-30.

Brown, G.C., and V. Borutaite. 2012. There is no evidence that mitochondria are the main source of reactive oxygen species in mammalian cells. Mitochondrion. 12:1-4.

Bruick, R.K. 2000. Expression of the gene encoding the proapoptotic Nip3 protein is induced by hypoxia. Proc Natl Acad Sci U S A. 97:9082-7.

Bruick, R.K., and S.L. McKnight. 2001. A conserved family of prolyl-4-hydroxylases that modify HIF. Science. 294:1337-40.

Burke, B., A. Giannoudis, K.P. Corke, D. Gill, M. Wells, L. Ziegler-Heitbrock, and C.E. Lewis. 2003. Hypoxia-induced gene expression in human macrophages: implications for ischemic tissues and hypoxia-regulated gene therapy. Am J Pathol. 163:1233-43.

Camp, V., and P. Martin. 1996. The role of macrophages in clearing programmed cell death in the developing kidney. Anat Embryol (Berl). 194:341-8.

Carreau, A., B. El Hafny-Rahbi, A. Matejuk, C. Grillon, and C. Kieda. 2011. Why is the partial oxygen pressure of human tissues a crucial parameter? Small molecules and hypoxia. J Cell Mol Med. 15:1239-53.

Chan, D.A., T.L. Kawahara, P.D. Sutphin, H.Y. Chang, J.T. Chi, and A.J. Giaccia. 2009. Tumor vasculature is regulated by PHD2-mediated angiogenesis and bone marrowderived cell recruitment. Cancer Cell. 15:527-38.

Chan, D.A., P.D. Sutphin, S.E. Yen, and A.J. Giaccia. 2005. Coordinate regulation of the oxygen-dependent degradation domains of hypoxia-inducible factor 1 alpha. Mol Cell Biol. 25:6415-26.

Chandel, N.S., D.S. McClintock, C.E. Feliciano, T.M. Wood, J.A. Melendez, A.M. Rodriguez, and P.T. Schumacker. 2000. Reactive oxygen species generated at mitochondrial complex III stabilize hypoxia-inducible factor-1alpha during hypoxia: a mechanism of O2 sensing. J Biol Chem. 275:25130-8.

Chen, E.Y., M. Fujinaga, and A.J. Giaccia. 1999. Hypoxic microenvironment within an embryo induces apoptosis and is essential for proper morphological development. Teratology. 60:215-25.

Chen, H.C. 2005. Boyden chamber assay. Methods Mol Biol. 294:15-22.

Choi, J.H., Y.B. Lee, J. Jung, S.G. Hwang, I.H. Oh, and G.J. Kim. 2015. Hypoxia Inducible Factor-1alpha Regulates the Migration of Bone Marrow Mesenchymal Stem Cells via Integrin alpha 4. Stem Cells Int. 2016:7932185. 
Cioffi, C.L., X.Q. Liu, P.A. Kosinski, M. Garay, and B.R. Bowen. 2003. Differential regulation of HIF-1 alpha prolyl-4-hydroxylase genes by hypoxia in human cardiovascular cells. Biochem Biophys Res Commun. 303:947-53.

Clausen, B.E., C. Burkhardt, W. Reith, R. Renkawitz, and I. Forster. 1999. Conditional gene targeting in macrophages and granulocytes using LysMcre mice. Transgenic Res. 8:265-77.

Counts, D.F., G.J. Cardinale, and S. Udenfriend. 1978. Prolyl hydroxylase half reaction: peptidyl prolyl-independent decarboxylation of alpha-ketoglutarate. Proc Natl Acad Sci U S A. 75:2145-9.

Cramer, T., Y. Yamanishi, B.E. Clausen, I. Forster, R. Pawlinski, N. Mackman, V.H. Haase, R. Jaenisch, M. Corr, V. Nizet, G.S. Firestein, H.P. Gerber, N. Ferrara, and R.S. Johnson. 2003. HIF-1alpha is essential for myeloid cell-mediated inflammation. Cell. 112:645-57.

Dashty, M. 2013. A quick look at biochemistry: carbohydrate metabolism. Clin Biochem. 46:1339-52.

del Peso, L., M.C. Castellanos, E. Temes, S. Martin-Puig, Y. Cuevas, G. Olmos, and M.O. Landazuri. 2003. The von Hippel Lindau/hypoxia-inducible factor (HIF) pathway regulates the transcription of the HIF-proline hydroxylase genes in response to low oxygen. J Biol Chem. 278:48690-5.

Dengler, V.L., M.D. Galbraith, and J.M. Espinosa. 2014. Transcriptional regulation by hypoxia inducible factors. Crit Rev Biochem Mol Biol. 49:1-15.

Dunwoodie, S.L. 2009. The role of hypoxia in development of the Mammalian embryo. Dev Cell. 17:755-73.

Earle, W.R., E.L. Schilling, and E. Shelton. 1950. Production of malignancy in vitro. X. Continued description of cells at the glass interface of the cultures. J Natl Cancer Inst. 10:1067-1103.

Ebert, B.L., J.D. Firth, and P.J. Ratcliffe. 1995. Hypoxia and mitochondrial inhibitors regulate expression of glucose transporter-1 via distinct Cis-acting sequences. J Biol Chem. 270:29083-9.

Epstein, A.C., J.M. Gleadle, L.A. McNeill, K.S. Hewitson, J. O'Rourke, D.R. Mole, M. Mukherji, E. Metzen, M.I. Wilson, A. Dhanda, Y.M. Tian, N. Masson, D.L. Hamilton, P. Jaakkola, R. Barstead, J. Hodgkin, P.H. Maxwell, C.W. Pugh, C.J. Schofield, and P.J. Ratcliffe. 2001. C. elegans EGL-9 and mammalian homologs define a family of dioxygenases that regulate HIF by prolyl hydroxylation. Cell. 107:43-54.

Firth, J.D., B.L. Ebert, and P.J. Ratcliffe. 1995. Hypoxic regulation of lactate dehydrogenase A. Interaction between hypoxia-inducible factor 1 and cAMP response elements. $J$ Biol Chem. 270:21021-7.

Forsythe, J.A., B.H. Jiang, N.V. Iyer, F. Agani, S.W. Leung, R.D. Koos, and G.L. Semenza. 1996. Activation of vascular endothelial growth factor gene transcription by hypoxiainducible factor 1. Mol Cell Biol. 16:4604-13.

Fukuda, R., H. Zhang, J.W. Kim, L. Shimoda, C.V. Dang, and G.L. Semenza. 2007. HIF-1 regulates cytochrome oxidase subunits to optimize efficiency of respiration in hypoxic cells. Cell. 129:111-22.

Galvan-Pena, S., and L.A. O'Neill. 2014. Metabolic reprograming in macrophage polarization. Front Immunol. 5:420.

Garvey, C., B. Tiep, R. Carter, M. Barnett, M. Hart, and R. Casaburi. 2012. Severe exerciseinduced hypoxemia. Respir Care. 57:1154-60.

Geissmann, F., M.G. Manz, S. Jung, M.H. Sieweke, M. Merad, and K. Ley. 2010. Development of monocytes, macrophages, and dendritic cells. Science. 327:656-61. 
Goda, N., and M. Kanai. 2012. Hypoxia-inducible factors and their roles in energy metabolism. Int J Hematol. 95:457-63.

Gordon, S. 2016. Elie Metchnikoff, the Man and the Myth. J Innate Immun.

Gordon, S., and F.O. Martinez. 2010. Alternative activation of macrophages: mechanism and functions. Immunity. 32:593-604.

Goren, I., N. Allmann, N. Yogev, C. Schurmann, A. Linke, M. Holdener, A. Waisman, J. Pfeilschifter, and S. Frank. 2009. A transgenic mouse model of inducible macrophage depletion: effects of diphtheria toxin-driven lysozyme M-specific cell lineage ablation on wound inflammatory, angiogenic, and contractive processes. Am J Pathol. 175:132-47.

Guzy, R.D., B. Hoyos, E. Robin, H. Chen, L. Liu, K.D. Mansfield, M.C. Simon, U. Hammerling, and P.T. Schumacker. 2005. Mitochondrial complex III is required for hypoxia-induced ROS production and cellular oxygen sensing. Cell Metab. 1:401-8.

Harbers, M. 2008. The current status of cDNA cloning. Genomics. 91:232-42.

Hayashi, M., M. Sakata, T. Takeda, T. Yamamoto, Y. Okamoto, K. Sawada, A. Kimura, R. Minekawa, M. Tahara, K. Tasaka, and Y. Murata. 2004. Induction of glucose transporter 1 expression through hypoxia-inducible factor 1alpha under hypoxic conditions in trophoblast-derived cells. $J$ Endocrinol. 183:145-54.

Heerlein, K., A. Schulze, L. Hotz, P. Bartsch, and H. Mairbaurl. 2005. Hypoxia decreases cellular ATP demand and inhibits mitochondrial respiration of a549 cells. Am J Respir Cell Mol Biol. 32:44-51.

Hewitson, K.S., L.A. McNeill, M.V. Riordan, Y.M. Tian, A.N. Bullock, R.W. Welford, J.M. Elkins, N.J. Oldham, S. Bhattacharya, J.M. Gleadle, P.J. Ratcliffe, C.W. Pugh, and C.J. Schofield. 2002. Hypoxia-inducible factor (HIF) asparagine hydroxylase is identical to factor inhibiting HIF (FIH) and is related to the cupin structural family. $J$ Biol Chem. 277:26351-5.

Hirsila, M., P. Koivunen, V. Gunzler, K.I. Kivirikko, and J. Myllyharju. 2003. Characterization of the human prolyl 4-hydroxylases that modify the hypoxiainducible factor. J Biol Chem. 278:30772-80.

Hoffman, D.L., J.D. Salter, and P.S. Brookes. 2007. Response of mitochondrial reactive oxygen species generation to steady-state oxygen tension: implications for hypoxic cell signaling. Am J Physiol Heart Circ Physiol. 292:H101-8.

Holmstrom, K.M., and T. Finkel. 2014. Cellular mechanisms and physiological consequences of redox-dependent signalling. Nat Rev Mol Cell Biol. 15:411-21.

Holscher, M., M. Silter, S. Krull, M. von Ahlen, A. Hesse, P. Schwartz, B. Wielockx, G. Breier, D.M. Katschinski, and A. Zieseniss. 2011. Cardiomyocyte-specific prolyl-4hydroxylase domain 2 knock out protects from acute myocardial ischemic injury. $J$ Biol Chem. 286:11185-94.

Huang, H., S. Van de Veire, M. Dalal, R. Parlier, R.D. Semba, P. Carmeliet, and S.A. Vinores. 2011. Reduced retinal neovascularization, vascular permeability, and apoptosis in ischemic retinopathy in the absence of prolyl hydroxylase-1 due to the prevention of hyperoxia-induced vascular obliteration. Invest Ophthalmol Vis Sci. 52:7565-73.

Huang, J., Q. Zhao, S.M. Mooney, and F.S. Lee. 2002. Sequence determinants in hypoxiainducible factor-1alpha for hydroxylation by the prolyl hydroxylases PHD1, PHD2, and PHD3. J Biol Chem. 277:39792-800.

Huang, L.E., J. Gu, M. Schau, and H.F. Bunn. 1998. Regulation of hypoxia-inducible factor 1alpha is mediated by an O2-dependent degradation domain via the ubiquitinproteasome pathway. Proc Natl Acad Sci U S A. 95:7987-92. 
Hume, D.A. 2015. The Many Alternative Faces of Macrophage Activation. Front Immunol. $6: 370$.

Ikeda, J., T. Ichiki, H. Matsuura, E. Inoue, J. Kishimoto, A. Watanabe, C. Sankoda, S. Kitamoto, T. Tokunou, K. Takeda, G.H. Fong, and K. Sunagawa. 2013. Deletion of phd 2 in myeloid lineage attenuates hypertensive cardiovascular remodeling. $J \mathrm{Am}$ Heart Assoc. 2:e00178.

Imhof, B.A., and M. Aurrand-Lions. 2004. Adhesion mechanisms regulating the migration of monocytes. Nat Rev Immunol. 4:432-44.

Ivan, M., T. Haberberger, D.C. Gervasi, K.S. Michelson, V. Gunzler, K. Kondo, H. Yang, I. Sorokina, R.C. Conaway, J.W. Conaway, and W.G. Kaelin, Jr. 2002. Biochemical purification and pharmacological inhibition of a mammalian prolyl hydroxylase acting on hypoxia-inducible factor. Proc Natl Acad Sci U S A. 99:13459-64.

Iyer, N.V., L.E. Kotch, F. Agani, S.W. Leung, E. Laughner, R.H. Wenger, M. Gassmann, J.D. Gearhart, A.M. Lawler, A.Y. Yu, and G.L. Semenza. 1998. Cellular and developmental control of $\mathrm{O} 2$ homeostasis by hypoxia-inducible factor 1 alpha. Genes Dev. 12:149-62.

Jaakkola, P., D.R. Mole, Y.M. Tian, M.I. Wilson, J. Gielbert, S.J. Gaskell, A. von Kriegsheim, H.F. Hebestreit, M. Mukherji, C.J. Schofield, P.H. Maxwell, C.W. Pugh, and P.J. Ratcliffe. 2001. Targeting of HIF-alpha to the von Hippel-Lindau ubiquitylation complex by O2-regulated prolyl hydroxylation. Science. 292:468-72.

Jaroszeski, M.J., and G. Radcliff. 1999. Fundamentals of flow cytometry. Mol Biotechnol. 11:37-53.

Jiang, B.H., J.Z. Zheng, S.W. Leung, R. Roe, and G.L. Semenza. 1997. Transactivation and inhibitory domains of hypoxia-inducible factor 1alpha. Modulation of transcriptional activity by oxygen tension. J Biol Chem. 272:19253-60.

Jurgensen, J.S., C. Rosenberger, M.S. Wiesener, C. Warnecke, J.H. Horstrup, M. Grafe, S. Philipp, W. Griethe, P.H. Maxwell, U. Frei, S. Bachmann, R. Willenbrock, and K.U. Eckardt. 2004. Persistent induction of HIF-1alpha and -2alpha in cardiomyocytes and stromal cells of ischemic myocardium. FASEB J. 18:1415-7.

Kaelin, W.G., Jr., and P.J. Ratcliffe. 2008. Oxygen sensing by metazoans: the central role of the HIF hydroxylase pathway. Mol Cell. 30:393-402.

Kallio, P.J., K. Okamoto, S. O'Brien, P. Carrero, Y. Makino, H. Tanaka, and L. Poellinger. 1998. Signal transduction in hypoxic cells: inducible nuclear translocation and recruitment of the $\mathrm{CBP} / \mathrm{p} 300$ coactivator by the hypoxia-inducible factor-1alpha. EMBO J. 17:6573-86.

Kalucka, J., A. Ettinger, K. Franke, S. Mamlouk, R.P. Singh, K. Farhat, A. Muschter, S. Olbrich, G. Breier, D.M. Katschinski, W. Huttner, A. Weidemann, and B. Wielockx. 2013. Loss of epithelial hypoxia-inducible factor prolyl hydroxylase 2 accelerates skin wound healing in mice. Mol Cell Biol. 33:3426-38.

Kato, M., J. Li, J.L. Chuang, and D.T. Chuang. 2007. Distinct structural mechanisms for inhibition of pyruvate dehydrogenase kinase isoforms by AZD7545, dichloroacetate, and radicicol. Structure. 15:992-1004.

Kaufmann, P., K. Engelstad, Y. Wei, S. Jhung, M.C. Sano, D.C. Shungu, W.S. Millar, X. Hong, C.L. Gooch, X. Mao, J.M. Pascual, M. Hirano, P.W. Stacpoole, S. DiMauro, and D.C. De Vivo. 2006. Dichloroacetate causes toxic neuropathy in MELAS: a randomized, controlled clinical trial. Neurology. 66:324-30.

Kawano, Y., J. Nakae, N. Watanabe, S. Fujisaka, K. Iskandar, R. Sekioka, Y. Hayashi, K. Tobe, M. Kasuga, T. Noda, A. Yoshimura, M. Onodera, and H. Itoh. 2012. Loss of 
Pdk1-Foxo1 signaling in myeloid cells predisposes to adipose tissue inflammation and insulin resistance. Diabetes. 61:1935-48.

Ke, Q., and M. Costa. 2006. Hypoxia-inducible factor-1 (HIF-1). Mol Pharmacol. 70:146980.

Kent, B.D., P.D. Mitchell, and W.T. McNicholas. 2011. Hypoxemia in patients with COPD: cause, effects, and disease progression. Int J Chron Obstruct Pulmon Dis. 6:199-208.

Kerbey, A.L., P.J. Randle, R.H. Cooper, S. Whitehouse, H.T. Pask, and R.M. Denton. 1976. Regulation of pyruvate dehydrogenase in rat heart. Mechanism of regulation of proportions of dephosphorylated and phosphorylated enzyme by oxidation of fatty acids and ketone bodies and of effects of diabetes: role of coenzyme A, acetylcoenzyme A and reduced and oxidized nicotinamide-adenine dinucleotide. Biochem J. 154:327-48.

Kim, J.W., I. Tchernyshyov, G.L. Semenza, and C.V. Dang. 2006. HIF-1-mediated expression of pyruvate dehydrogenase kinase: a metabolic switch required for cellular adaptation to hypoxia. Cell Metab. 3:177-85.

Kiss, J., M. Mollenhauer, S.R. Walmsley, J. Kirchberg, P. Radhakrishnan, T. Niemietz, J. Dudda, G. Steinert, M.K. Whyte, P. Carmeliet, M. Mazzone, J. Weitz, and M. Schneider. 2012. Loss of the oxygen sensor PHD3 enhances the innate immune response to abdominal sepsis. J Immunol. 189:1955-65.

Knowles, H.J., R.R. Raval, A.L. Harris, and P.J. Ratcliffe. 2003. Effect of ascorbate on the activity of hypoxia-inducible factor in cancer cells. Cancer Res. 63:1764-8.

Koh, M.Y., R. Lemos, Jr., X. Liu, and G. Powis. 2011. The hypoxia-associated factor switches cells from HIF-1alpha- to HIF-2alpha-dependent signaling promoting stem cell characteristics, aggressive tumor growth and invasion. Cancer Res. 71:4015-27.

Koivunen, P., P. Tiainen, J. Hyvarinen, K.E. Williams, R. Sormunen, S.J. Klaus, K.I. Kivirikko, and J. Myllyharju. 2007. An endoplasmic reticulum transmembrane prolyl 4-hydroxylase is induced by hypoxia and acts on hypoxia-inducible factor alpha. $J$ Biol Chem. 282:30544-52.

Kolobova, E., A. Tuganova, I. Boulatnikov, and K.M. Popov. 2001. Regulation of pyruvate dehydrogenase activity through phosphorylation at multiple sites. Biochem J. 358:6977.

Korotchkina, L.G., and M.S. Patel. 1995. Mutagenesis studies of the phosphorylation sites of recombinant human pyruvate dehydrogenase. Site-specific regulation. J Biol Chem. 270:14297-304.

Laitala, A., E. Aro, G. Walkinshaw, J.M. Maki, M. Rossi, M. Heikkila, E.R. Savolainen, M. Arend, K.I. Kivirikko, P. Koivunen, and J. Myllyharju. 2012. Transmembrane prolyl 4-hydroxylase is a fourth prolyl 4-hydroxylase regulating EPO production and erythropoiesis. Blood. 120:3336-44.

Lando, D., D.J. Peet, J.J. Gorman, D.A. Whelan, M.L. Whitelaw, and R.K. Bruick. 2002. FIH-1 is an asparaginyl hydroxylase enzyme that regulates the transcriptional activity of hypoxia-inducible factor. Genes Dev. 16:1466-71.

Laurindo, F.R., D.C. Fernandes, and C.X. Santos. 2008. Assessment of superoxide production and NADPH oxidase activity by HPLC analysis of dihydroethidium oxidation products. Methods Enzymol. 441:237-60.

Lee, Y.M., C.H. Jeong, S.Y. Koo, M.J. Son, H.S. Song, S.K. Bae, J.A. Raleigh, H.Y. Chung, M.A. Yoo, and K.W. Kim. 2001. Determination of hypoxic region by hypoxia marker in developing mouse embryos in vivo: a possible signal for vessel development. Dev Dyn. 220:175-86. 
Lewis, J.S., J.A. Lee, J.C. Underwood, A.L. Harris, and C.E. Lewis. 1999. Macrophage responses to hypoxia: relevance to disease mechanisms. J Leukoc Biol. 66:889-900.

Li, F., Z.Z. Chong, and K. Maiese. 2004. Erythropoietin on a tightrope: balancing neuronal and vascular protection between intrinsic and extrinsic pathways. Neurosignals. 13:265-89.

Lieb, M.E., K. Menzies, M.C. Moschella, R. Ni, and M.B. Taubman. 2002. Mammalian EGLN genes have distinct patterns of mRNA expression and regulation. Biochem Cell Biol. 80:421-6.

Linn, T.C., F.H. Pettit, and L.J. Reed. 1969. Alpha-keto acid dehydrogenase complexes. X. Regulation of the activity of the pyruvate dehydrogenase complex from beef kidney mitochondria by phosphorylation and dephosphorylation. Proc Natl Acad Sci U S A. 62:234-41.

Liu, J., H. Wang, and J. Li. 2016. Inflammation and Inflammatory Cells in Myocardial Infarction and Reperfusion Injury: A Double-Edged Sword. Clin Med Insights Cardiol. 10:79-84.

Liu, W., S.M. Shen, X.Y. Zhao, and G.Q. Chen. 2012. Targeted genes and interacting proteins of hypoxia inducible factor-1. Int J Biochem Mol Biol. 3:165-78.

Liu, Y., S.R. Cox, T. Morita, and S. Kourembanas. 1995. Hypoxia regulates vascular endothelial growth factor gene expression in endothelial cells. Identification of a 5' enhancer. Circ Res. 77:638-43.

Loenarz, C., M.L. Coleman, A. Boleininger, B. Schierwater, P.W. Holland, P.J. Ratcliffe, and C.J. Schofield. 2011. The hypoxia-inducible transcription factor pathway regulates oxygen sensing in the simplest animal, Trichoplax adhaerens. EMBO Rep. 12:63-70.

Macdougall, I.C. 2012. New anemia therapies: translating novel strategies from bench to bedside. Am J Kidney Dis. 59:444-51.

Mahon, P.C., K. Hirota, and G.L. Semenza. 2001. FIH-1: a novel protein that interacts with HIF-1alpha and VHL to mediate repression of HIF-1 transcriptional activity. Genes Dev. 15:2675-86.

Maiuri, M.C., G. Le Toumelin, A. Criollo, J.C. Rain, F. Gautier, P. Juin, E. Tasdemir, G. Pierron, K. Troulinaki, N. Tavernarakis, J.A. Hickman, O. Geneste, and G. Kroemer. 2007. Functional and physical interaction between $\mathrm{Bcl}-\mathrm{X}(\mathrm{L})$ and a $\mathrm{BH} 3$-like domain in Beclin-1. EMBO J. 26:2527-39.

Makino, Y., R. Uenishi, K. Okamoto, T. Isoe, O. Hosono, H. Tanaka, A. Kanopka, L. Poellinger, M. Haneda, and C. Morimoto. 2007. Transcriptional up-regulation of inhibitory PAS domain protein gene expression by hypoxia-inducible factor 1 (HIF-1): a negative feedback regulatory circuit in HIF-1-mediated signaling in hypoxic cells. $J$ Biol Chem. 282:14073-82.

Mamlouk, S., J. Kalucka, R.P. Singh, K. Franke, A. Muschter, A. Langer, C. Jakob, M. Gassmann, G.B. Baretton, and B. Wielockx. 2014. Loss of prolyl hydroxylase-2 in myeloid cells and T-lymphocytes impairs tumor development. Int J Cancer. 134:84958.

Mansfield, K.D., R.D. Guzy, Y. Pan, R.M. Young, T.P. Cash, P.T. Schumacker, and M.C. Simon. 2005. Mitochondrial dysfunction resulting from loss of cytochrome c impairs cellular oxygen sensing and hypoxic HIF-alpha activation. Cell Metab. 1:393-9.

Marxsen, J.H., P. Stengel, K. Doege, P. Heikkinen, T. Jokilehto, T. Wagner, W. Jelkmann, P. Jaakkola, and E. Metzen. 2004. Hypoxia-inducible factor-1 (HIF-1) promotes its degradation by induction of HIF-alpha-prolyl-4-hydroxylases. Biochem J. 381:761-7.

Maxwell, P.H., and K.U. Eckardt. 2015. HIF prolyl hydroxylase inhibitors for the treatment of renal anaemia and beyond. Nat Rev Nephrol. 12:157-68. 
Maxwell, P.H., M.S. Wiesener, G.W. Chang, S.C. Clifford, E.C. Vaux, M.E. Cockman, C.C. Wykoff, C.W. Pugh, E.R. Maher, and P.J. Ratcliffe. 1999. The tumour suppressor protein VHL targets hypoxia-inducible factors for oxygen-dependent proteolysis. Nature. 399:271-5.

Maynard, M.A., A.J. Evans, T. Hosomi, S. Hara, M.A. Jewett, and M. Ohh. 2005. Human HIF-3alpha4 is a dominant-negative regulator of HIF-1 and is down-regulated in renal cell carcinoma. FASEB J. 19:1396-406.

Mazzone, M., D. Dettori, R. Leite de Oliveira, S. Loges, T. Schmidt, B. Jonckx, Y.M. Tian, A.A. Lanahan, P. Pollard, C. Ruiz de Almodovar, F. De Smet, S. Vinckier, J. Aragones, K. Debackere, A. Luttun, S. Wyns, B. Jordan, A. Pisacane, B. Gallez, M.G. Lampugnani, E. Dejana, M. Simons, P. Ratcliffe, P. Maxwell, and P. Carmeliet. 2009. Heterozygous Deficiency of PHD2 Restores Tumor Oxygenation and Inhibits Metastasis via Endothelial Normalization. Cell.

McDonough, M.A., V. Li, E. Flashman, R. Chowdhury, C. Mohr, B.M. Lienard, J. Zondlo, N.J. Oldham, I.J. Clifton, J. Lewis, L.A. McNeill, R.J. Kurzeja, K.S. Hewitson, E. Yang, S. Jordan, R.S. Syed, and C.J. Schofield. 2006. Cellular oxygen sensing: Crystal structure of hypoxia-inducible factor prolyl hydroxylase (PHD2). Proc Natl Acad Sci U S A. 103:9814-9.

Meiser, J., L. Kramer, S.C. Sapcariu, N. Battello, J. Ghelfi, A.F. D'Herouel, A. Skupin, and K. Hiller. 2015. Pro-inflammatory Macrophages Sustain Pyruvate Oxidation through Pyruvate Dehydrogenase for the Synthesis of Itaconate and to Enable Cytokine Expression. J Biol Chem. 291:3932-46.

Melillo, G., T. Musso, A. Sica, L.S. Taylor, G.W. Cox, and L. Varesio. 1995. A hypoxiaresponsive element mediates a novel pathway of activation of the inducible nitric oxide synthase promoter. J Exp Med. 182:1683-93.

Metzen, E., U. Berchner-Pfannschmidt, P. Stengel, J.H. Marxsen, I. Stolze, M. Klinger, W.Q. Huang, C. Wotzlaw, T. Hellwig-Burgel, W. Jelkmann, H. Acker, and J. Fandrey. 2003. Intracellular localisation of human HIF-1 alpha hydroxylases: implications for oxygen sensing. J Cell Sci. 116:1319-26.

Metzen, E., D.P. Stiehl, K. Doege, J.H. Marxsen, T. Hellwig-Burgel, and W. Jelkmann. 2005. Regulation of the prolyl hydroxylase domain protein 2 (phd2/egln-1) gene: identification of a functional hypoxia-responsive element. Biochem J. 387:711-7.

Michelakis, E.D., L. Webster, and J.R. Mackey. 2008. Dichloroacetate (DCA) as a potential metabolic-targeting therapy for cancer. Br J Cancer. 99:989-94.

Mills, E.L., and L.A. O'Neill. 2016. Reprogramming mitochondrial metabolism in macrophages as an anti-inflammatory signal. Eur J Immunol. 46:13-21.

Minamishima, Y.A., J. Moslehi, N. Bardeesy, D. Cullen, R.T. Bronson, and W.G. Kaelin, Jr. 2008. Somatic inactivation of the PHD2 prolyl hydroxylase causes polycythemia and congestive heart failure. Blood. 111:3236-44.

Minamishima, Y.A., J. Moslehi, R.F. Padera, R.T. Bronson, R. Liao, and W.G. Kaelin, Jr. 2009. A feedback loop involving the Phd3 prolyl hydroxylase tunes the mammalian hypoxic response in vivo. Mol Cell Biol. 29:5729-41.

Mirza, R., L.A. DiPietro, and T.J. Koh. 2009. Selective and specific macrophage ablation is detrimental to wound healing in mice. Am J Pathol. 175:2454-62.

Mittal, M., M. Roth, P. Konig, S. Hofmann, E. Dony, P. Goyal, A.C. Selbitz, R.T. Schermuly, H.A. Ghofrani, G. Kwapiszewska, W. Kummer, W. Klepetko, M.A. Hoda, L. Fink, J. Hanze, W. Seeger, F. Grimminger, H.H. Schmidt, and N. Weissmann. 2007. Hypoxiadependent regulation of nonphagocytic NADPH oxidase subunit NOX4 in the pulmonary vasculature. Circ Res. 101:258-67. 
Mollenhauer, M., J. Kiss, J. Dudda, J. Kirchberg, N. Rahbari, P. Radhakrishnan, T. Niemietz, V. Rausch, J. Weitz, and M. Schneider. 2012. Deficiency of the oxygen sensor PHD1 augments liver regeneration after partial hepatectomy. Langenbecks Arch Surg. 397:1313-22.

Mora, A., A.M. Davies, L. Bertrand, I. Sharif, G.R. Budas, S. Jovanovic, V. Mouton, C.R. Kahn, J.M. Lucocq, G.A. Gray, A. Jovanovic, and D.R. Alessi. 2003. Deficiency of PDK1 in cardiac muscle results in heart failure and increased sensitivity to hypoxia. EMBO J. 22:4666-76.

Moroz, E., S. Carlin, K. Dyomina, S. Burke, H.T. Thaler, R. Blasberg, and I. Serganova. 2009. Real-time imaging of HIF-1alpha stabilization and degradation. PLoS One. 4:e5077.

Mosser, D.M., and J.P. Edwards. 2008. Exploring the full spectrum of macrophage activation. Nat Rev Immunol. 8:958-69.

Nahrendorf, M., M.J. Pittet, and F.K. Swirski. 2011. Monocytes: protagonists of infarct inflammation and repair after myocardial infarction. Circulation. 121:2437-45.

Nietfeld, J.J., and A. Kemp. 1981. The function of ascorbate with respect to prolyl 4hydroxylase activity. Biochim Biophys Acta. 657:159-67.

Obach, M., A. Navarro-Sabate, J. Caro, X. Kong, J. Duran, M. Gomez, J.C. Perales, F. Ventura, J.L. Rosa, and R. Bartrons. 2004. 6-Phosphofructo-2-kinase (pfkfb3) gene promoter contains hypoxia-inducible factor-1 binding sites necessary for transactivation in response to hypoxia. J Biol Chem. 279:53562-70.

Ohh, M., C.W. Park, M. Ivan, M.A. Hoffman, T.Y. Kim, L.E. Huang, N. Pavletich, V. Chau, and W.G. Kaelin. 2000. Ubiquitination of hypoxia-inducible factor requires direct binding to the beta-domain of the von Hippel-Lindau protein. Nat Cell Biol. 2:423-7.

Paky, A., J.R. Michael, T.M. Burke-Wolin, M.S. Wolin, and G.H. Gurtner. 1993. Endogenous production of superoxide by rabbit lungs: effects of hypoxia or metabolic inhibitors. $J$ Appl Physiol (1985). 74:2868-74.

Papandreou, I., R.A. Cairns, L. Fontana, A.L. Lim, and N.C. Denko. 2006. HIF-1 mediates adaptation to hypoxia by actively downregulating mitochondrial oxygen consumption. Cell Metab. 3:187-97.

Pause, A., B. Peterson, G. Schaffar, R. Stearman, and R.D. Klausner. 1999. Studying interactions of four proteins in the yeast two-hybrid system: structural resemblance of the $\mathrm{pVHL/elongin} \mathrm{BC/hCUL-2} \mathrm{complex} \mathrm{with} \mathrm{the} \mathrm{ubiquitin} \mathrm{ligase} \mathrm{complex}$ SKP1/cullin/F-box protein. Proc Natl Acad Sci U S A. 96:9533-8.

Peng, J., L. Zhang, L. Drysdale, and G.H. Fong. 2000. The transcription factor EPAS1/hypoxia-inducible factor 2alpha plays an important role in vascular remodeling. Proc Natl Acad Sci U S A. 97:8386-91.

Pescador, N., Y. Cuevas, S. Naranjo, M. Alcaide, D. Villar, M.O. Landazuri, and L. Del Peso. 2005. Identification of a functional hypoxia-responsive element that regulates the expression of the egl nine homologue 3 (egln3/phd3) gene. Biochem J. 390:189-97.

Peyssonnaux, C., V. Datta, T. Cramer, A. Doedens, E.A. Theodorakis, R.L. Gallo, N. Hurtado-Ziola, V. Nizet, and R.S. Johnson. 2005. HIF-1 alpha expression regulates the bactericidal capacity of phagocytes. J Clin Invest. 115:1806-15.

Promega. Celltiter glo online: https://www.promega.de/ /media/files/resources/protocols/technical\%20bulletins/0/ce lltiter\%20glo\%20luminescent\%20cell\%20viability\%20assay\%20protocol.pdf, retrieved 18.07.2016. 
Pugh, C.W., J.F. O'Rourke, M. Nagao, J.M. Gleadle, and P.J. Ratcliffe. 1997. Activation of hypoxia-inducible factor-1; definition of regulatory domains within the alpha subunit. J Biol Chem. 272:11205-14.

R-Biopharm. L-Milchsäure Bestimmungskit. online: http://www.r-biopharm.com/wpcontent/uploads/4050/Lactic-acid-L_DE_10139084035_2013-03.pdf, retrieved 18.07.2016.

Raes, G., P. De Baetselier, W. Noel, A. Beschin, F. Brombacher, and G. Hassanzadeh Gh. 2002. Differential expression of FIZZ1 and Ym1 in alternatively versus classically activated macrophages. J Leukoc Biol. 71:597-602.

Raheja, L.F., D.C. Genetos, A. Wong, and C.E. Yellowley. 2011. Hypoxic regulation of mesenchymal stem cell migration: the role of RhoA and HIF-1alpha. Cell Biol Int. 35:981-9.

Rardin, M.J., S.E. Wiley, R.K. Naviaux, A.N. Murphy, and J.E. Dixon. 2009. Monitoring phosphorylation of the pyruvate dehydrogenase complex. Anal Biochem. 389:157-64.

Raschke, W.C., S. Baird, P. Ralph, and I. Nakoinz. 1978. Functional macrophage cell lines transformed by Abelson leukemia virus. Cell. 15:261-7.

Riboldi, E., C. Porta, S. Morlacchi, A. Viola, A. Mantovani, and A. Sica. 2013. Hypoxiamediated regulation of macrophage functions in pathophysiology. Int Immunol. 25:6775.

Ridley, A.J. 2015. Rho GTPase signalling in cell migration. Curr Opin Cell Biol. 36:103-12.

Ryan, H.E., J. Lo, and R.S. Johnson. 1998. HIF-1 alpha is required for solid tumor formation and embryonic vascularization. EMBO J. 17:3005-15.

Schioppa, T., B. Uranchimeg, A. Saccani, S.K. Biswas, A. Doni, A. Rapisarda, S. Bernasconi, S. Saccani, M. Nebuloni, L. Vago, A. Mantovani, G. Melillo, and A. Sica. 2003.

Regulation of the chemokine receptor CXCR4 by hypoxia. J Exp Med. 198:1391-402.

Schneider, M., K. Van Geyte, P. Fraisl, J. Kiss, J. Aragones, M. Mazzone, H. Mairbaurl, K. De Bock, N.H. Jeoung, M. Mollenhauer, M. Georgiadou, T. Bishop, C. Roncal, A. Sutherland, B. Jordan, B. Gallez, J. Weitz, R.A. Harris, P. Maxwell, M. Baes, P. Ratcliffe, and P. Carmeliet. 2010. Loss or silencing of the PHD1 prolyl hydroxylase protects livers of mice against ischemia/reperfusion injury. Gastroenterology. 138:1143-54 e1-2.

Schofield, C.J., and P.J. Ratcliffe. 2004. Oxygen sensing by HIF hydroxylases. Nat Rev Mol Cell Biol. 5:343-54.

SeahorseBioscience. Glycostress Test. online: http://www.seahorsebio.com/resources/pdfs/manual-xf96-glycolysis-stress-test-kit.pdf, retrieved 18.07.2016..

SeahorseBioscience. Mito Stress Test. online: http://www.seahorsebio.com/support/software/PDF/XF_Cell_Mito_Stress_Test_Kit_U ser_Guide.pdf, retrieved 18.07.2016.

Semba, H., N. Takeda, T. Isagawa, Y. Sugiura, K. Honda, M. Wake, H. Miyazawa, Y. Yamaguchi, M. Miura, D.M. Jenkins, H. Choi, J.W. Kim, M. Asagiri, A.S. Cowburn, H. Abe, K. Soma, K. Koyama, M. Katoh, K. Sayama, N. Goda, R.S. Johnson, I. Manabe, R. Nagai, and I. Komuro. 2016. HIF-1alpha-PDK1 axis-induced active glycolysis plays an essential role in macrophage migratory capacity. Nat Commun. 7:11635.

Semenza, G.L. 2004. Hydroxylation of HIF-1: oxygen sensing at the molecular level. Physiology (Bethesda). 19:176-82.

Semenza, G.L. 2010. Vascular responses to hypoxia and ischemia. Arterioscler Thromb Vasc Biol. 30:648-52. 
Semenza, G.L. 2014. Hypoxia-inducible factor 1 and cardiovascular disease. Anпи Rev Physiol. 76:39-56.

Semenza, G.L., B.H. Jiang, S.W. Leung, R. Passantino, J.P. Concordet, P. Maire, and A. Giallongo. 1996. Hypoxia response elements in the aldolase A, enolase 1, and lactate dehydrogenase A gene promoters contain essential binding sites for hypoxia-inducible factor 1. J Biol Chem. 271:32529-37.

Semenza, G.L., P.H. Roth, H.M. Fang, and G.L. Wang. 1994. Transcriptional regulation of genes encoding glycolytic enzymes by hypoxia-inducible factor 1. J Biol Chem. 269:23757-63.

Semenza, G.L., and G.L. Wang. 1992. A nuclear factor induced by hypoxia via de novo protein synthesis binds to the human erythropoietin gene enhancer at a site required for transcriptional activation. Mol Cell Biol. 12:5447-54.

Shirato, K., T. Kizaki, T. Sakurai, J.E. Ogasawara, Y. Ishibashi, T. Iijima, C. Okada, I. Noguchi, K. Imaizumi, N. Taniguchi, and H. Ohno. 2009. Hypoxia-inducible factor1alpha suppresses the expression of macrophage scavenger receptor 1. Pflugers Arch. 459:93-103.

Sica, A., and A. Mantovani. 2012. Macrophage plasticity and polarization: in vivo veritas. $J$ Clin Invest. 122:787-95.

Sigma-Aldrich. PDH activity assay. online: http://www.sigmaaldrich.com/content/dam/sigmaaldrich/docs/Sigma/Bulletin/1/mak18 3bul.pdf, retrieved: 18.07.2016.

Singh, R.P., K. Franke, J. Kalucka, S. Mamlouk, A. Muschter, A. Gembarska, T. Grinenko, C. Willam, R. Naumann, K. Anastassiadis, A.F. Stewart, S. Bornstein, T. Chavakis, G. Breier, C. Waskow, and B. Wielockx. 2013. HIF prolyl hydroxylase 2 (PHD2) is a critical regulator of hematopoietic stem cell maintenance during steady-state and stress. Blood. 121:5158-66.

Smirnova, N.A., D.M. Hushpulian, R.E. Speer, I.N. Gaisina, R.R. Ratan, and I.G. Gazaryan. 2012. Catalytic mechanism and substrate specificity of HIF prolyl hydroxylases. Biochemistry (Mosc). 77:1108-19.

Stacpoole, P.W. 1989. The pharmacology of dichloroacetate. Metabolism. 38:1124-44.

Stacpoole, P.W., D.S. Kerr, C. Barnes, S.T. Bunch, P.R. Carney, E.M. Fennell, N.M. Felitsyn, R.L. Gilmore, M. Greer, G.N. Henderson, A.D. Hutson, R.E. Neiberger, R.G. O'Brien, L.A. Perkins, R.G. Quisling, A.L. Shroads, J.J. Shuster, J.H. Silverstein, D.W. Theriaque, and E. Valenstein. 2006. Controlled clinical trial of dichloroacetate for treatment of congenital lactic acidosis in children. Pediatrics. 117:1519-31.

Sugden, M.C., and M.J. Holness. 2003. Recent advances in mechanisms regulating glucose oxidation at the level of the pyruvate dehydrogenase complex by PDKs. Am J Physiol Endocrinol Metab. 284:E855-62.

Suhara, T., T. Hishiki, M. Kasahara, N. Hayakawa, T. Oyaizu, T. Nakanishi, A. Kubo, H. Morisaki, W.G. Kaelin, Jr., M. Suematsu, and Y.A. Minamishima. 2016. Inhibition of the oxygen sensor PHD2 in the liver improves survival in lactic acidosis by activating the Cori cycle. Proc Natl Acad Sci U S A. 112:11642-7.

Swain, L., M. Wottawa, A. Hillemann, A. Beneke, H. Odagiri, K. Terada, M. Endo, Y. Oike, K. Farhat, and D.M. Katschinski. 2013. Prolyl-4-hydroxylase domain 3 (PHD3) is a critical terminator for cell survival of macrophages under stress conditions. In $\mathrm{J}$ Leukoc Biol. Vol. 96. 365-75.

Takeda, K., A. Cowan, and G.H. Fong. 2007. Essential role for prolyl hydroxylase domain protein 2 in oxygen homeostasis of the adult vascular system. Circulation. 116:77481. 
Takeda, K., V.C. Ho, H. Takeda, L.J. Duan, A. Nagy, and G.H. Fong. 2006. Placental but not heart defects are associated with elevated hypoxia-inducible factor $\alpha$ levels in mice lacking prolyl hydroxylase domain protein 2. Mol Cell Biol. 26:8336-46.

Takeda, Y., S. Costa, E. Delamarre, C. Roncal, R. Leite de Oliveira, M.L. Squadrito, V. Finisguerra, S. Deschoemaeker, F. Bruyere, M. Wenes, A. Hamm, J. Serneels, J. Magat, T. Bhattacharyya, A. Anisimov, B.F. Jordan, K. Alitalo, P. Maxwell, B. Gallez, Z.W. Zhuang, Y. Saito, M. Simons, M. De Palma, and M. Mazzone. Macrophage skewing by Phd2 haplodeficiency prevents ischaemia by inducing arteriogenesis. Nature. 479:122-6.

Takeda, Y., S. Costa, E. Delamarre, C. Roncal, R. Leite de Oliveira, M.L. Squadrito, V. Finisguerra, S. Deschoemaeker, F. Bruyere, M. Wenes, A. Hamm, J. Serneels, J. Magat, T. Bhattacharyya, A. Anisimov, B.F. Jordan, K. Alitalo, P. Maxwell, B. Gallez, Z.W. Zhuang, Y. Saito, M. Simons, M. De Palma, and M. Mazzone. 2011. Macrophage skewing by Phd 2 haplodeficiency prevents ischaemia by inducing arteriogenesis. Nature. 479:122-6.

Tambuwala, M.M., E.P. Cummins, C.R. Lenihan, J. Kiss, M. Stauch, C.C. Scholz, P. Fraisl, F. Lasitschka, M. Mollenhauer, S.P. Saunders, P.H. Maxwell, P. Carmeliet, P.G. Fallon, M. Schneider, and C.T. Taylor. 2010. Loss of prolyl hydroxylase-1 protects against colitis through reduced epithelial cell apoptosis and increased barrier function. Gastroenterology. 139:2093-101.

Tan, Z., N. Xie, H. Cui, D.R. Moellering, E. Abraham, V.J. Thannickal, and G. Liu. 2015. Pyruvate dehydrogenase kinase 1 participates in macrophage polarization via regulating glucose metabolism. J Immunol. 194:6082-9.

Tanimoto, K., Y. Makino, T. Pereira, and L. Poellinger. 2000. Mechanism of regulation of the hypoxia-inducible factor-1 alpha by the von Hippel-Lindau tumor suppressor protein. EMBO J. 19:4298-309.

Taylor, C.T. 2008. Mitochondria and cellular oxygen sensing in the HIF pathway. Biochem J. 409:19-26.

Thompson, A.A., J. Binham, T. Plant, M.K. Whyte, and S.R. Walmsley. 2013. Hypoxia, the HIF pathway and neutrophilic inflammatory responses. Biol Chem. 394:471-7.

Tian, H., R.E. Hammer, A.M. Matsumoto, D.W. Russell, and S.L. McKnight. 1998. The hypoxia-responsive transcription factor EPAS1 is essential for catecholamine homeostasis and protection against heart failure during embryonic development. Genes Dev. 12:3320-4.

Tuckerman, J.R., Y. Zhao, K.S. Hewitson, Y.M. Tian, C.W. Pugh, P.J. Ratcliffe, and D.R. Mole. 2004. Determination and comparison of specific activity of the HIF-prolyl hydroxylases. FEBS Lett. 576:145-50.

Turner, L., C. Scotton, R. Negus, and F. Balkwill. 1999. Hypoxia inhibits macrophage migration. Eur J Immunol. 29:2280-7.

Urao, N., R.D. McKinney, T. Fukai, and M. Ushio-Fukai. 2012. NADPH oxidase 2 regulates bone marrow microenvironment following hindlimb ischemia: role in reparative mobilization of progenitor cells. Stem Cells. 30:923-34.

Vaupel, P., and A. Mayer. 2007. Hypoxia in cancer: significance and impact on clinical outcome. Cancer Metastasis Rev. 26:225-39.

Vogler, M., S. Vogel, S. Krull, K. Farhat, P. Leisering, S. Lutz, C.M. Wuertz, D.M. Katschinski, and A. Zieseniss. 2013. Hypoxia modulates fibroblastic architecture, adhesion and migration: a role for HIF-1alpha in cofilin regulation and cytoplasmic actin distribution. PLoS One. 8:e69128. 
Vogler, M., A. Zieseniss, A.R. Hesse, E. Levent, M. Tiburcy, E. Heinze, N. Burzlaff, G. Schley, K.U. Eckardt, C. Willam, and D.M. Katschinski. 2015. Pre- and postconditional inhibition of prolyl-4-hydroxylase domain enzymes protects the heart from an ischemic insult. Pflugers Arch. 467:2141-9.

Walmsley, S.R., E.R. Chilvers, A.A. Thompson, K. Vaughan, H.M. Marriott, L.C. Parker, G. Shaw, S. Parmar, M. Schneider, I. Sabroe, D.H. Dockrell, M. Milo, C.T. Taylor, R.S. Johnson, C.W. Pugh, P.J. Ratcliffe, P.H. Maxwell, P. Carmeliet, and M.K. Whyte. 2011. Prolyl hydroxylase 3 (PHD3) is essential for hypoxic regulation of neutrophilic inflammation in humans and mice. J Clin Invest. 121:1053-63.

Wang, G.L., B.H. Jiang, E.A. Rue, and G.L. Semenza. 1995. Hypoxia-inducible factor 1 is a basic-helix-loop-helix-PAS heterodimer regulated by cellular $\mathrm{O} 2$ tension. Proc Natl Acad Sci U S A. 92:5510-4.

Wang, J., Z. Hong, C. Zeng, Q. Yu, and H. Wang. 2014. NADPH oxidase 4 promotes cardiac microvascular angiogenesis after hypoxia/reoxygenation in vitro. Free Radic Biol Med. 69:278-88.

Warburg, O., F. Wind, and E. Negelein. 1927. The Metabolism of Tumors in the Body. J Gen Physiol. 8:519-30.

Weinberg, J.M., M.A. Venkatachalam, N.F. Roeser, and I. Nissim. 2000. Mitochondrial dysfunction during hypoxia/reoxygenation and its correction by anaerobic metabolism of citric acid cycle intermediates. Proc Natl Acad Sci U S A. 97:2826-31.

Weischenfeldt, J., and B. Porse. 2008. Bone Marrow-Derived Macrophages (BMM): Isolation and Applications. CSH Protoc. 2008:pdb prot5080.

Wenger, R.H., I. Kvietikova, A. Rolfs, M. Gassmann, and H.H. Marti. 1997. Hypoxiainducible factor-1 alpha is regulated at the post-mRNA level. Kidney Int. 51:560-3.

Wilhelm, J., and A. Pingoud. 2003. Real-time polymerase chain reaction. Chembiochem. 4:1120-8.

Wilkins, M.R., H.A. Ghofrani, N. Weissmann, A. Aldashev, and L. Zhao. 2015. Pathophysiology and treatment of high-altitude pulmonary vascular disease. Circulation. 131:582-90.

Wong, B.W., A. Kuchnio, U. Bruning, and P. Carmeliet. 2013. Emerging novel functions of the oxygen-sensing prolyl hydroxylase domain enzymes. Trends Biochem Sci. 38:311.

Xie, L., X. Pi, Z. Wang, J. He, M.S. Willis, and C. Patterson. 2015. Depletion of PHD3 protects heart from ischemia/reperfusion injury by inhibiting cardiomyocyte apoptosis. J Mol Cell Cardiol. 80:156-65.

Young, R.K., R.M. Cailleau, B. Mackay, and W.J. Reeves. 1974. Establishment of epithelial cell line MDA-MB-157 from metastatic pleural effusion of human breast carcinoma. In Vitro. 9:239-45.

Zhang, H., M. Bosch-Marce, L.A. Shimoda, Y.S. Tan, J.H. Baek, J.B. Wesley, F.J. Gonzalez, and G.L. Semenza. 2008. Mitochondrial autophagy is an HIF-1-dependent adaptive metabolic response to hypoxia. J Biol Chem. 283:10892-903.

Zhao, J., J. Zhang, M. Yu, Y. Xie, Y. Huang, D.W. Wolff, P.W. Abel, and Y. Tu. 2013. Mitochondrial dynamics regulates migration and invasion of breast cancer cells. Oncogene. 32:4814-24. 


\section{Vielen Dank!}

First and foremost, I would like to express my gratitude to my supervisor Prof. Dörthe Katschinski. Thank you for having given me the opportunity to work on this interesting project, for your guidance, advice and support and especially for sometimes reassuring me and for telling me that everything will be fine.

I would like to thank my co-supervisor Prof. Ajay Shah for his insightful advice and guidance. I really appreciate that the time you took to discuss my work and for your great input to the project. Thank you for having me in your laboratory!

Many thanks go to Celio X. Santos for his kind supervision during my stay in the Shah lab and for making the transition smooth and comfortable.

Furthermore, I would also like to thank the members of my thesis committee Prof. Susanne Lutz und Prof. Frauke Alves for their continuous support and advice throughout my project.

Many thanks as well to Prof. Holger Reichardt and Prof. Ralf Dressel for kindly agreeing to be on my examination committee.

My sincere thanks also go to my lab mates! Thank you for creating such a warm and productive work environment. Thanks for your support and care which helped me to overcome setbacks and to remain focused! Thanks to Marieke for supporting me especially during the first months in the lab. Thanks also to Annette, Angelika, Shun and Anke for their experimental support. I would also like to express my gratitude to Dörthe, Anke and Bert for the incredible fast and thorough correction of this thesis and for their helpful comments and suggestions.

Thanks as well to the department of Biochemistry (laboratory of Prof. Rehling), especially Dr. Jan Dudek for teaching me how to isolate mitochondria and how to measure oxygen consumption. Thanks as well for always staying interested in the project and that I could always come to you with questions and discuss the obtained data.

I am also very grateful for my great friends. Thanks to the "Schopis" Max, Thaddäus und Franziska. We probably had one of the most amazing and relaxed flat shares ever. Thanks to Elena, Angelika, Janine and Laura who shared my ups and downs. Thanks also to Chris for being supportive and understanding. 
Furthermore, I would like to express my gratitude to my family. None of this would have been possible without their love, their understanding and their strength. Thank you for supporting me in my rather "exotic" career choice, even though it is probably still a mystery to you what I do all day. Thanks for believing in me and for sometimes calming down and telling me that everything will be fine. Thanks for understanding when I was very busy and forgot to call or if I could not make it to family celebrations. Thank you for giving me the security that there is always someone behind me and that I am not alone. 